\title{
On Character Sheaves and Characters of Reductive Groups at Unipotent Classes
}

\author{
François Digne, Gustav Lehrer, and Jean Michel
}

\begin{abstract}
With a view to determining character values of finite reductive groups at unipotent elements, we prove a number of results concerning inner products of generalised Gelfand-Graev characters with characteristic functions of character sheaves, here called Lusztig functions. These are used to determine projections of generalised Gelfand-Graev characters to the space of unipotent characters, and to the space of characters with a given wave front set. Such projections are expressed largely in terms of Weyl group data. We show how the values of characters at their unipotent support or wave front set are determined by such data. In some exceptional groups we show that the projection of a generalised Gelfand-Graev character to a family with the same wave front set is (up to sign) the dual of a Mellin transform. Using these results, in certain cases we are able to determine roots of unity which relate almost characters to the characteristic functions. In particular we show how to compute the values of all unipotent characters at all unipotent classes for the exceptional groups of type $G_{2}, F_{4}, E_{6}$, ${ }^{2} E_{6}, E_{7}$ and $E_{8}$ by a method different from that of [L86, K2]; we therefore require weaker restrictions on $p$ and $q$. We also provide an appendix which gives a complete list of the cuspidal character sheaves on all quasi-simple groups.
\end{abstract}

Keywords: Reductive group, character sheaf, Gelfand-Graev.

\section{Introduction}

Let $\mathbf{G}$ be a connected reductive algebraic group over a field of positive characteristic $p$. We shall generally assume that $p$ is "sufficiently large", which will often mean "larger than the Coxeter number of the associated Weyl group". Let $F$ be a Frobenius morphism defining a rational structure on $\mathbf{G}$ over the finite extension $\mathbb{F}_{q}$ of the finite field $\mathbb{F}_{p}$ with $p$ elements. We

Received March 13, 2014.

2010 Mathematics Subject Classification.Primary 20C33; secondary 20G05, $20 \mathrm{G} 40$. 
shall be be concerned with the irreducible characters of the finite group of fixed points $\mathbf{G}^{F}$ over the field $\overline{\mathbb{Q}}_{\ell}$, where $\ell$ is a prime different from $p$. The purpose of this work is to contribute to the determination of the values of the irreducible characters of $\mathbf{G}^{F}$ at unipotent elements of $\mathbf{G}^{F}$. In addition, we include, as an appendix (A) a classification of the cuspidal character sheaves for quasi simple groups $\mathbf{G}$, which is complete up to a small number of ambiguities. This classification is essentially due to Lusztig [L85, but we provide a list, conveniently arranged, of cuspidal character sheaves for each isogeny type of quasi-simple group. Specifically, we give a discussion of the

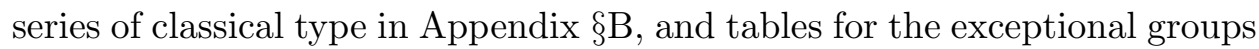
in Appendix C.

The irreducible characters of $\mathbf{G}^{F}$ are partitioned into subsets in various ways. The cuspidal character sheaves on Levi subgroups of $\mathbf{G}$ lead to a classification into "Harish-Chandra type" series, whose constituents are labelled by (twisted) characters of an appropriate Coxeter group. Each character of $\mathbf{G}^{F}$ has a wave front set and a unipotent support, both of which are (geometric) unipotent conjugacy classes of $\mathbf{G}$, and belongs to a 'Lusztig series'. The Lusztig series are further partitioned into families. Our results relate principally to certain classes of characters which are described in terms of these partitions.

The notion of determination of a value requires explanation. Lusztig has shown that the space $\mathcal{C}\left(\mathbf{G}^{F}\right)$ of class functions on $\mathbf{G}^{F}$ has an orthonormal basis consisting of the characteristic functions of $F$-invariant simple G-equivariant perverse sheaves on $\mathbf{G}$. Such functions will be referred to as Lusztig functions, and we consider their values known by the work of Lusztig [L85. Further, it was shown in [S1, S2, S3, S4, B, W] that with certain qualifications, the Lusztig functions coincide with 'almost characters' (see \$6) up to multiplication by a root of unity. Since the transition from almost characters to irreducible characters is known, it follows that determination of this root of unity implies the determination of the values of certain characters.

Our main results are as follows.

In $\$ 2$ we complement DLM3 by giving an expression for the characteristic function of an arbitrary unipotent class in terms of duals of generalised Gelfand-Graev characters, and as a consequence deduce some results concerning the support of these duals. The special cases of the regular and subregular classes are spelled out explicitly. We also give a formula for the value of any irreducible character at any element of its unipotent support. In $\$ 3$, we provide some background on character sheaves and families, and define Lusztig series and the wave front set in a way suitable for our purpose. In $\$ 3.4$ we determine the multiplicity of an irreducible character $\chi$ of $\mathbf{G}^{F}$ in 
the Mellin transform $\Gamma_{\iota}$ of the generalised Gelfand-Graev characters, when $\iota$ is in the principal series, in terms of the Lusztig series of $\chi$. This is applied to special cases such as subregular $\chi$, where explicit formulae may be given.

Section 4 provides a general study of the restriction to the unipotent set of Lusztig functions, which are defined as characteristic functions of $F$ stable character sheaves, and applications to the determination of various multiplicities. The restriction to $\mathbf{G}_{\text {uni }}^{F}$ of the Lusztig function $\chi_{E, \phi_{E}}$ (see $\$ 3.2$ ) is given (Theorem 4.1 and Lemma 4.6) in terms of Weyl group data and Green functions. This is applied in Theorem 4.9 to give the inner product of a Lusztig function with a generalised Gelfand-Graev character. These results are applied to prove vanishing theorems for inner products, and in Corollary 4.18 to give an explicit expression for the projection of $\Gamma_{\iota}$ to the space spanned by the characters with given wave front set in terms of Weyl group data. In $\$ 5$ this is applied to show that when the wave front set above is the support of $\iota$, then the projection to the unipotent characters of the above projection is precisely a Lusztig function, up to sign. This explains phenomena which had been observed in several examples earlier. We also show how the inner product of any irreducible character with $\Gamma_{\iota}$ may be expressed in terms of its inner products with Lusztig functions.

Section 6 treats a special situation which applies in the cases when $\mathbf{G}$ is of type $G_{2}, F_{4}$ or $E_{8}$. In this situation, we are able to determine the root of unity which relates the almost characters to the Lusztig functions, by an analysis which uses the Mellin transforms (see Definition 6.3) of the characters in a family. Specifically we compute ( $c f$. Theorem 6.5) the projection of a generalised Gelfand-Graev character to the space space spanned by a family with the same wave front set. In the last paragraph of $\$ 6.1$ it is explained how this permits the computation of character values.

Finally, as mentioned above, in Appendices $\mathrm{A} B \mathrm{~B}$ and $\mathrm{C}$, we present a complete classification of the cuspidal character sheaves on all quasi-simple groups $\mathbf{G}$.

\section{Characteristic functions and generalised Gelfand-Graev Characters.}

We maintain the notation of [DLM3], which we now briefly review. Consider pairs $\iota=(C, \zeta)$, where $C$ is a unipotent class of $\mathbf{G}$ and $\zeta$ is a $\mathbf{G}$ equivariant irreducible $\overline{\mathbb{Q}}_{\ell}$-local system on $C ; C$ is said to be the support of the pair and may also be written $C_{\iota}$. Set $a_{\iota}=|A(u)|$ for $u \in C$, where $A(u)=C_{\mathbf{G}}(u) / C_{\mathbf{G}}^{0}(u)$. Each such pair belongs to a cuspidal pair on a Levi subgroup $\mathbf{L}$ of $\mathbf{G}$, and all pairs belonging to a given cuspidal system form 
a block $\mathcal{I}$; we also denote $\mathbf{L}$ by $\mathbf{L}_{\mathcal{I}}$. When $\mathcal{I}$ is $F$-stable we may choose the

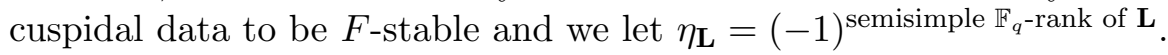

Suppose $X$ is an algebraic variety over $\overline{\mathbb{F}}_{q}$, with an $\mathbb{F}_{q}$-structure embodied in the Frobenius morphism $F: X \rightarrow X$. If $\chi$ is a complex of $\overline{\mathbb{Q}}_{\ell}$-sheaves on $X$ and we are given an isomorphism $\phi: F^{*} \chi \stackrel{\sim}{\longrightarrow} \chi$, then this data permits the definition (see [DLM3, §2]) of a function $c_{\phi}: X^{F} \rightarrow \overline{\mathbb{Q}}_{\ell}$, called the characteristic function of the pair $\chi, \phi$, where as usual, $X^{F}$ denotes the set of $F$-stable points of $X$. For an $F$-stable pair $\iota$, we denote by $\mathcal{Y}_{\iota}$ the characteristic function of $\zeta$ and by $\mathcal{X}_{\iota}$ the characteristic function of the corresponding intersection cohomology complex. Writing $c_{\iota}=\frac{1}{2}\left(\operatorname{codim} C-\operatorname{dim} Z_{\mathbf{L}}\right)$, we define the normalisations $\widetilde{\mathcal{Y}}_{\iota}=q^{c_{\iota}} \mathcal{Y}_{\iota}$ and $\widetilde{\mathcal{X}}_{\iota}=q^{c_{\iota}} \mathcal{X}_{\iota}$ of $\mathcal{Y}_{\iota}$ and $\mathcal{I}_{\iota}$ respectively. Define a partial order on pairs by stipulating $\iota \leq \kappa$ if the pairs are in the same block and $C_{\iota} \subset \bar{C}_{\kappa}$. We have $\mathcal{X}_{\iota}=\sum_{\kappa \leq \iota} P_{\kappa, \iota} \mathcal{Y}_{\kappa}$ for some $P_{\kappa, \iota} \in \mathbb{Z}[q]$; we define $P_{\kappa, \iota}=0$ when $\kappa \not \leq \iota$, and the normalised version $\tilde{P}_{\kappa, \iota}=q^{c_{\iota}-c_{\kappa}} P_{\kappa, \iota}$ so that $\widetilde{\mathcal{X}}_{\iota}=\sum_{\kappa<\iota} \widetilde{P}_{\kappa, \iota} \widetilde{\mathcal{Y}}_{\kappa}$.

The fixed point set $C^{F}$ splits into $\mathbf{G}^{F}$-classes indexed by the set $H^{1}(F, A(u))$ of $F$-classes in $A(u)$, where $u$ is any (chosen) element of $C^{F}$. For $a \in A(u)$ we denote by $u_{a}$ a representative of the $\mathbf{G}^{F}$-class defined by the $F$-class of $a$, and denote by $\Gamma_{u_{a}}$ the Generalised Gelfand-Graev character attached to the $\mathbf{G}^{F}$-class of $u_{a}$. We let $\Gamma_{\iota}=\sum_{a \in A(u)} \mathcal{Y}_{\iota}\left(u_{a}\right) \Gamma_{u_{a}}$ and define the normalisation $\widetilde{\Gamma}_{\iota}=a_{\iota}^{-1} \zeta_{\mathcal{I}} \Gamma_{\iota}$, where $\zeta_{\mathcal{I}}$ is the fourth root of unity attached by Lusztig to a block $\mathcal{I}$ (see [L92b, 7.2]). Finally we denote by $D$ (or $D_{\mathbf{G}}$ when appropriate) the Alvis-Curtis duality operation on $\mathcal{C}\left(\mathbf{G}^{F}\right)$ and by $f \mapsto f^{*}$ the operation on Laurent polynomials in $q$ such that $f^{*}(q)=f\left(q^{-1}\right)$. Note that we refer to the $\Gamma_{\iota}$ as the 'Mellin transforms' of the $\Gamma_{u_{a}}$; this should not be confused with the 'Mellin transforms' defined in Definition 6.3 below.

For $\mathcal{I}$ an $F$-stable block the sets $\left(\mathcal{Y}_{\iota}\right)_{\iota \in \mathcal{I}^{F}},\left(\mathcal{X}_{\iota}\right)_{\iota \in \mathcal{I}^{F}}$ and $\left(\Gamma_{\iota}\right)_{\iota \in \mathcal{I}^{F}}$ are three bases of the same space $\mathcal{C}_{\mathcal{I}}\left(\mathbf{G}^{F}\right)$ of unipotently supported class functions. This space is stable under Alvis-Curtis duality and two such spaces attached to different blocks are orthogonal.

Let $\chi_{(x)}$ be the normalised characteristic function of the class in $\mathbf{G}^{F}$ of an element $x$, i.e., the function whose value is zero outside this class and $\left|C_{\mathbf{G}}(x)^{F}\right|$ on the class. The following result is a variation on $[\mathrm{G}$, Lemma 2.5, Cor 2.6], which we shall require below. 
Theorem 2.1. Let $u$ be a rational unipotent element. Then the normalised characteristic function of the $\mathbf{G}^{F}$-class of $u$ is given by

$$
\chi_{(u)}=\sum_{\mathcal{I}} \eta_{\mathbf{L}_{\mathcal{I}}} \zeta_{\mathcal{I}} \sum_{\iota \in \mathcal{I}^{F}} \overline{\widetilde{\mathcal{Y}}_{\iota}(u)} \sum_{\substack{\gamma \geq \iota \\ \gamma \in \mathcal{I}^{F}}} a_{\gamma}^{-1}\left(\widetilde{P}\left(\widetilde{P}^{*}\right)^{-1}\right)_{\iota, \gamma} D \Gamma_{\gamma}
$$

where $\mathcal{I}$ runs over the rational blocks and $\widetilde{P}$ is the matrix whose $(\iota, \gamma)$ entry is the polynomial $\widetilde{P}_{\iota, \gamma}$.

Note that the sum in the theorem is over those blocks which contain a local system whose support is the class of $u$.

Proof. We shall find coefficients $m_{\iota, \gamma}$ such that the set of functions $\left(f_{\iota}=\right.$ $\left.\sum_{\gamma} m_{\iota, \gamma} D \Gamma_{\gamma}\right)$ is the basis of the space $\mathcal{C}_{\text {uni }}\left(\mathbf{G}^{F}\right)$ of unipotently supported class functions dual to the basis $\left(\widetilde{\mathcal{Y}}_{\iota}\right)$ for the usual inner product $\left\langle f, f^{\prime}\right\rangle_{\mathbf{G}^{F}}$ on class functions. It will then follow that $\chi_{(u)}=\sum_{\iota} \widetilde{\mathcal{Y}}_{\iota}(u) f_{\iota}=$ $\sum_{\iota} \widehat{\widetilde{\mathcal{Y}}_{\iota}(u)} \sum_{\gamma} m_{\iota, \gamma} D \Gamma_{\gamma}$.

The coefficients $m_{\iota, \gamma}$ are determined by the equations $\sum_{\gamma} m_{\iota, \gamma}\left\langle D \Gamma_{\gamma}, \widetilde{\mathcal{Y}}_{\lambda}\right\rangle_{\mathbf{G}^{F}}=\delta_{\iota, \lambda}$. By orthogonality of the spaces $\mathcal{C}_{\mathcal{I}}\left(\mathbf{G}^{F}\right)$ for different blocks, we have $m_{\iota, \gamma}=0$ unless $\iota$ and $\gamma$ are in the same block. For any total order extending $\leq$ the matrix $\tilde{P}_{\iota, \gamma}$ is upper unitriangular, thus invertible; using $\widetilde{\mathcal{Y}}_{\lambda}=\sum_{\kappa \in \mathcal{I}} \tilde{P}_{\kappa, \lambda}^{\prime} \widetilde{\mathcal{X}}_{\kappa}$ where $\tilde{P}_{\kappa, \lambda}^{\prime}$ are the entries of the matrix $\widetilde{P}^{-1}$, we get $\left\langle D \Gamma_{\gamma}, \widetilde{\mathcal{Y}}_{\lambda}\right\rangle_{\mathbf{G}^{F}}=\sum_{\kappa} \tilde{P}_{\kappa, \lambda}^{\prime}\left\langle D \Gamma_{\gamma}, \widetilde{\mathcal{X}}_{\kappa}\right\rangle_{\mathbf{G}^{F}}$ if $\lambda$ and $\gamma$ are in the same block $\mathcal{I}$, and the inner product is 0 otherwise. So by [DLM3, proof of 6.2] we get $\left\langle D \Gamma_{\gamma}, \widetilde{\mathcal{Y}}_{\lambda}\right\rangle_{\mathbf{G}^{F}}=\sum_{\kappa} \tilde{P}_{\kappa, \lambda}^{\prime} \eta_{\mathbf{L}_{\mathcal{I}}} a_{\gamma} \zeta_{\mathcal{I}}^{-1} \tilde{P}_{\gamma, \kappa}^{*}$ and the equations for the $m_{\iota, \gamma}$, when $\iota$ and $\gamma$ are in a block $\mathcal{I}$, are $\sum_{\gamma} m_{\iota, \gamma} \eta_{\mathbf{L}_{\mathcal{I}}} a_{\gamma} \zeta_{\mathcal{I}}^{-1} \sum_{\kappa} \tilde{P}_{\kappa, \lambda}^{\prime} \tilde{P}_{\gamma, \kappa}^{*}=\delta_{\iota, \lambda}$. As $\sum_{\kappa} \tilde{P}_{\kappa, \lambda}^{\prime} \tilde{P}_{\gamma, \kappa}^{*}$ is the $(\gamma, \lambda)$ entry of $\widetilde{P}^{*} \widetilde{P}^{-1}$, this can be written in matrix terms as follows, $M$ being the matrix with entries $m_{\iota, \gamma}$ :

$$
M\left(\begin{array}{lll}
\ddots & & \\
& a_{\gamma} & \\
& & \ddots
\end{array}\right) \widetilde{P}^{*} \widetilde{P}^{-1}=\eta_{\mathbf{L}_{\mathcal{I}} \zeta_{\mathcal{I}} I},
$$

where $I$ is the unit matrix. Hence

$$
m_{\iota, \gamma}=a_{\gamma}^{-1} \eta_{\mathbf{L}_{\mathcal{I}}} \zeta_{\mathcal{I}}\left(\widetilde{P}\left(\widetilde{P}^{*}\right)^{-1}\right)_{\iota, \gamma},
$$

as stated. 
Scholium 2.3. For any rational unipotent element $u$ the (virtual) character $D_{G} \Gamma_{u}$ is supported by unipotent classes greater than or equal to the class of $u$.

Proof. Let $C$ be the geometric class of $u$. The statement is equivalent to the analogous support property for the functions $D_{G}\left(\Gamma_{\iota}\right)$ for all $\iota$ supported by $C$. Using the triangular shape of the matrices $\left(\widetilde{P}_{\kappa, \lambda}^{\prime}\right)$ and $\left(\widetilde{P}_{\kappa, \lambda}^{*}\right)$ it follows from the proof of Theorem 2.1, that $\left\langle D \Gamma_{\gamma}, \widetilde{\mathcal{Y}}_{\lambda}\right\rangle_{\mathbf{G}^{F}}$ is zero unless $\gamma \leq \lambda$. Since the characteristic function of the $G^{F}$-class of a unipotent element $u$ is a linear combination of $\widetilde{\mathcal{Y}}_{\lambda}$ with $\lambda$ running over pairs with support $C$, the result follows.

The last statement is also a consequence of the following result.

Corollary 2.4. With notation as in Theorem 2.1, we have

$$
\chi_{(u)}=\sum_{\left\{C \subseteq \mathbf{G}_{u n i} \mid u \in \bar{C}\right\}}|A(C)|^{-1} \sum_{a \in A(C)} c\left(u, v_{a}\right) D_{\mathbf{G}} \Gamma_{v_{a}},
$$

where $C$ runs over the unipotent classes, $A(C)=A(v)$ for $v \in C$ and $v_{a} \in$ $C^{F}$ corresponds to $a \in A(C)$, and

$$
c\left(u, v_{a}\right)=\sum_{\mathcal{I} \in \mathcal{P}^{F}} \eta_{\mathbf{L}_{\mathcal{I}}} \zeta_{\mathcal{I}}^{-1} \widetilde{\mathcal{Y}}_{\mathcal{I}}(u)^{t} \widetilde{P}\left(\widetilde{P}^{*}\right)^{-1} \widetilde{\mathcal{Y}}_{\mathcal{I}}\left(v_{a}\right),
$$

and $\widetilde{\mathcal{Y}}_{\mathcal{I}}(u)$ denotes the column vector with entries $\widetilde{\mathcal{Y}}_{\iota}(u)$ where $\iota$ runs over $\mathcal{I}$.

Proof. We substitute the relation $\Gamma_{\gamma}=\sum_{a \in A(v)} \mathcal{Y}_{\gamma}\left(v_{a}\right) \Gamma_{v_{a}}$ into equation (2.2) and rearrange.

Scholium 2.3 follows from the above statement by simply inverting the equation for $\chi_{(u)}$.

Now we specialise Theorem 2.1 to the case of a regular unipotent class; let $\Gamma_{u}^{\mathcal{I}}$ be the orthogonal projection of $\Gamma_{u}$ onto the space $\mathcal{C}_{\mathcal{I}}\left(\mathbf{G}^{F}\right)$ and write $c_{\mathcal{I}}(u)$ for the common value of $c_{\iota}$ for $\iota \in \mathcal{I}$ whose support contains $u$ :

Corollary 2.6. If $u$ is a rational regular unipotent element, then

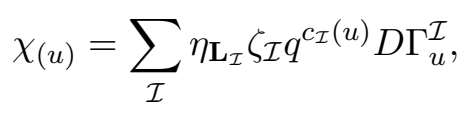

where the sum is over the regular blocks (those blocks containing a pair whose support is the regular unipotent class). 
Proof. Since the matrix $\widetilde{P}$ is unitriangular and the diagonal blocks attached to local systems with a given support are identity submatrices, formula 2.2 reduces to:

$$
\chi_{(u)}=\sum_{\mathcal{I}} \eta_{\mathbf{L}_{\mathcal{I}} \zeta_{\mathcal{I}}} \sum_{\left\{\iota \in \mathcal{I}^{F} \mid C_{\iota} \ni u\right\}} \overline{\widetilde{\mathcal{Y}}_{\iota}(u)} a_{\iota}^{-1} D \Gamma_{\iota} .
$$

Now substitute the value $\Gamma_{u}^{\mathcal{I}}=\sum_{\left\{\iota \in \mathcal{I}^{F} \mid C_{\iota} \ni u\right\}} \overline{\mathcal{Y}}_{\iota}(u) a_{\iota}^{-1} \Gamma_{\iota}$ given in DLM3, lemma 6.3] to obtain the result.

The corresponding result for subregular elements is

Corollary 2.7. If $u$ is a rational subregular unipotent element

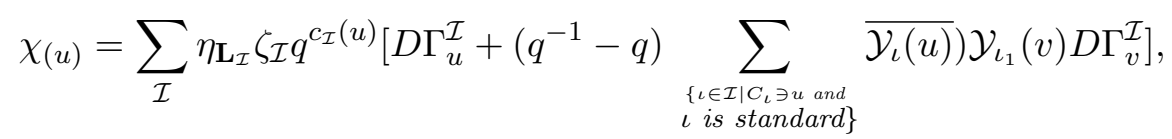

where $v$ is any regular rational unipotent element, $\iota_{1}$ is the pair in $\mathcal{I}$ labelled via the Springer correspondence by the trivial representation, and where "standard" has the sense of [DLM3, proposition 7.1].

The sum above may be restricted to the blocks which contain a local system supported by the subregular class.

Proof. Let $\iota$ be a pair with subregular support; [DLM3, proposition 7.1] states (once a misprint $q$ for $q^{-1}$ is corrected in (i)) that if $\iota$ is standard then $\widetilde{P}_{\iota, \gamma}=\left\{\begin{array}{ll}q^{-1} & \text { if } \gamma=\iota_{1} \\ \delta_{\iota, \gamma} & \text { otherwise }\end{array}\right.$ and otherwise $\widetilde{P}_{\iota, \gamma}=\delta_{\iota, \gamma}$. In the first case we can arrange the matrix $\widetilde{P}$ so that it is upper unitriangular and the lower right corner, indexed by $\iota$ and $\iota_{1}$, is $\left(\begin{array}{cc}1 & q^{-1} \\ 0 & 1\end{array}\right)$. In the second case we can arrange $\widetilde{P}$ so that it is upper unitriangular and $\iota$ indexes the last line. The lower right block of $\widetilde{P}\left(\widetilde{P}^{*}\right)^{-1}$ in the two respective cases is then $\left(\begin{array}{cc}1 & q^{-1}-q \\ 0 & 1\end{array}\right)$ or (1).

Using these values, formula 2.2 reduces to:

$$
\chi_{(u)}=\sum_{\mathcal{I}} \eta_{\mathbf{L}_{\mathcal{I}} \zeta_{\mathcal{I}}}\left(\sum_{\left\{\iota \in \mathcal{I}^{F} \mid C_{\iota} \ni u\right\}} \frac{\overline{\mathcal{\mathcal { Y }}_{\iota}(u)}}{a_{\iota}} D \Gamma_{\iota}+\sum_{\substack{\left\{\iota \in \mathcal{I} \mid C_{\iota} \ni u \text { and } \\ \iota \text { is standard }\right\}}} \frac{\overline{\mathcal{\mathcal { Y }}_{\iota}(u)}}{a_{\iota_{1}}}\left(q^{-1}-q\right) D \Gamma_{\iota_{1}}\right) .
$$

The first term in the sum can be transformed as in Corollary 2.6. If we take into account that there is at most one regularly supported local system 
in a block (see [DLM2, 1.10]), which in this case we take to be $\iota_{1}$, then $D \Gamma_{v}^{\mathcal{I}}=\overline{\mathcal{Y}}_{\iota_{1}}(v) a_{\iota_{1}}^{-1} D \Gamma_{\iota_{1}}$ which yields $a_{\iota_{1}}^{-1} D \Gamma_{\iota_{1}}=\mathcal{Y}_{\iota_{1}}(v) D \Gamma_{v}^{\mathcal{I}}$ since $\mathcal{Y}_{\iota_{1}}(v)$ is a root of unity because $A(v)$ is commutative. The second term is now as in the statement of the corollary .

We now look at the leading term in the formula $(2.2)$, i.e., the term indexed by $\gamma$ such that $u$ is in the support of $\gamma$.

Theorem 2.8. (cf. [G, §2.4]) If $v$ is a rational unipotent element and $\iota$ a rational pair such that $C_{\iota} \ni v$, we have

$$
D \Gamma_{\iota}(v)=|A(v)|\left|\left(C_{\mathbf{G}}(v)^{\circ}\right)^{F}\right| \eta_{\mathbf{L}_{\mathcal{I}}} \zeta_{\mathcal{I}}^{-1} q^{-c_{\iota}} \mathcal{Y}_{\iota}(v),
$$

where $\mathcal{I}$ denotes the block of $\iota$.

Proof. Assume that $v$ is a rational unipotent element in the same geometric class as $u$. Since in 2.2 the only terms which do not vanish are those where $C_{\iota} \ni u$, thus $C_{\iota} \ni v$, and since $D \Gamma_{\gamma}(v)=0$ when $\gamma>\iota$ and since the diagonal blocks in $\widetilde{P}$ pertaining to pairs with the same support are identity matrices, we get

$$
\chi_{(u)}(v)=\left|C_{\mathbf{G}^{F}}(u)\right| \delta_{(u),(v)}=\sum_{\mathcal{I}} \eta_{\mathbf{L}_{\mathcal{I}} \zeta_{\mathcal{I}}} \sum_{\iota \in \mathcal{I}^{F}} \overline{\widetilde{\mathcal{Y}}_{\iota}(u)} a_{\iota}^{-1} D \Gamma_{\iota}(v) .
$$

We now use the orthogonality relation [DLM3, (4.2)] for the $\mathcal{Y}_{\iota}$ which can be written

$$
\sum_{(u)}\left|A(u)^{F}\right|^{-1} \overline{\mathcal{Y}_{\iota}(u)} \mathcal{Y}_{\gamma}(u)=\delta_{\iota \cdot \gamma},
$$

where $(u)$ runs over the rational classes contained in $C_{\iota}$. Multiplying both sides of the rightmost equation in $(2.9)$ by $\left|A(u)^{F}\right|^{-1} \mathcal{Y}_{\gamma}(u)$ and summing over $(u)$ we get

$$
\left|A(v)^{F}\right|^{-1} \mathcal{Y}_{\gamma}(v)\left|C_{\mathbf{G}^{F}}(v)\right|=\eta_{\mathbf{L}_{\mathcal{I}}} \zeta_{\mathcal{I}} q^{c_{\gamma}} a_{\gamma}^{-1} D \Gamma_{\gamma}(v),
$$

whenever $C_{\gamma} \ni v$, whence the theorem.

The following corollary, which may be found in [G, 2.4(a)], is also a direct consequence of the formulas in the proof of Theorem 2.1.

Corollary 2.10. Let $\iota$ and $\gamma$ be two pairs with same support; then

$$
\left\langle D \Gamma_{\iota}, \mathcal{Y}_{\gamma}\right\rangle_{\mathbf{G}^{F}}= \begin{cases}0 & \text { if } \iota \neq \gamma \\ a_{\iota} \eta_{\mathbf{L}_{\mathcal{I}} \zeta_{\mathcal{I}}^{-1} q^{-c_{\iota}}} & \text { if } \iota=\gamma \in \mathcal{I}\end{cases}
$$


Proof. By Theorem 2.8 we have

$$
\begin{gathered}
\left\langle D \Gamma_{\iota}, \mathcal{Y}_{\gamma}\right\rangle_{\mathbf{G}^{F}}=\left|\mathbf{G}^{F}\right|^{-1} \sum_{v \in(\operatorname{supp} \iota)^{F}} D \Gamma_{\iota}(v) \overline{\mathcal{Y}_{\gamma}(v)}= \\
\left|\mathbf{G}^{F}\right|^{-1} \sum_{v \in(\operatorname{supp} \iota)^{F}}|A(v)|\left|A(v)^{F}\right|^{-1}\left|C_{\mathbf{G}^{F}}(v)\right| \eta_{\mathbf{L}_{\mathcal{I}}} \zeta_{\mathcal{I}}^{-1} q^{-c_{\iota}} \mathcal{Y}_{\iota}(v) \overline{\mathcal{Y}_{\gamma}(v)},
\end{gathered}
$$

where $\mathcal{I}$ is the block containing $\iota$. The last sum reduces to

$$
\sum_{a \in A(v)} \eta_{\mathbf{L}_{\mathcal{I}}} \zeta_{\mathcal{I}}^{-1} q^{-c_{\iota}} \mathcal{Y}_{\iota}\left(u_{a}\right) \overline{\mathcal{Y}_{\gamma}\left(u_{a}\right)}
$$

where $u_{a}$ is a representative of the rational conjugacy class in $C_{\iota}$ parameterised by $a$, given the choice of $v \in C_{\iota}$. Applying the orthogonality formula [DLM3, (4.2)], we obtain the result.

Remark 2.11. If we apply the above theorem to a regular unipotent element we recover formula [DLM2, 2.1].

Let us now compute the value of an irreducible character on its "unipotent support" (see Definition 3.5). The following proposition generalises [DLM1, 3.15.4].

Proposition 2.12. Let $\chi$ be an irreducible character and $v$ a rational unipotent element such that $\left\langle\chi, D \Gamma_{u}\right\rangle_{\mathbf{G}^{F}}=0$ for any $u$ in a conjugacy class strictly larger than the conjugacy class of $v$, then

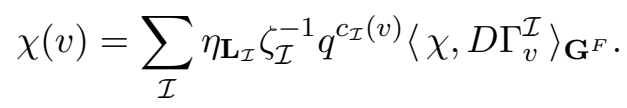

Proof. We have $\chi(v)=\left\langle\chi, \chi_{(v)}\right\rangle_{\mathbf{G}^{F}}$. By Theorem 2.1 this is equal to

$$
\sum_{\mathcal{I}} \eta_{\mathbf{L}_{\mathcal{I}}} \zeta_{\mathcal{I}}^{-1} \sum_{\iota \in \mathcal{I}^{F}} \tilde{\mathcal{Y}}_{\iota}(v) \sum_{\gamma \geq \iota} a_{\gamma}^{-1}\left(\widetilde{P}\left(\tilde{P}^{*}\right)^{-1}\right)_{\iota, \gamma}\left\langle\chi, D \Gamma_{\gamma}\right\rangle_{\mathbf{G}^{F}}
$$

In this formula, if $\iota \in \mathcal{I}^{F}$ yields a non-zero summand, then $C_{\iota} \ni v$ and as $\gamma \geq \iota$, the inner product in the sum is zero unless $\gamma=\iota$. Hence we have $\chi(v)=\sum_{\mathcal{I}} \eta_{\mathbf{L}_{\mathcal{I}}} \zeta_{\mathcal{I}}^{-1} \sum_{\iota \in \mathcal{I}^{F}} \tilde{\mathcal{Y}}_{\iota}(v) a_{\iota}^{-1}\left\langle\chi, D \Gamma_{\iota}\right\rangle_{\mathbf{G}^{F}}$, which can be written

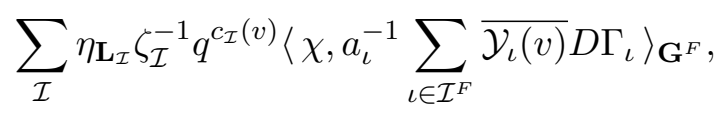

which completes the proof. 


\section{Character sheaves, wave front set, Lusztig series and Families}

We begin with some background.

\subsection{Character sheaves}

These arise as follows. Our notation is similar to that in $[\mathrm{L92b}$ and $\underline{\mathrm{AA}}$, with some significant departures. Let $\mathbf{L}$ be a Levi subgroup of $\mathbf{G}$ and let $\iota_{\overline{\mathbf{L}}}:=$ $(C, \xi)$ be a cuspidal local system in $\mathbf{L} / Z^{0}(\mathbf{L})$, where $C$ is a conjugacy class of the latter group and $\xi$ is a local system on $C$. Let $\overline{\mathcal{S}}$ be a Kummer local system on the torus $\mathbf{L} /[\mathbf{L}, \mathbf{L}]$. We may then form the local system $\iota_{\overline{\mathbf{L}}} \otimes \overline{\mathcal{S}}$ on $\mathbf{L} / Z^{0}(\mathbf{L}) \times \mathbf{L} /[\mathbf{L}, \mathbf{L}]$. The pullback of this local system under the map $\mathbf{L} \rightarrow$ $\mathbf{L} / Z^{0}(\mathbf{L}) \times \mathbf{L} /[\mathbf{L}, \mathbf{L}]$ is supported on $\Sigma:=Z^{0}(\mathbf{L}) C$, and we denote it by $\iota_{\mathbf{L}} \otimes$ $\mathcal{S}$, where $\iota_{\mathbf{L}}$ and $\mathcal{S}$ are respectively the pullbacks of $\iota_{\overline{\mathbf{L}}}$ and $\overline{\mathcal{S}}$. The intersection complex extension $\operatorname{IC}\left(\iota_{\mathbf{L}} \otimes \mathcal{S}\right)[\operatorname{dim} \Sigma]$ is then a cuspidal character sheaf [L85, 3.12] [L84b, 2.5], and it is known [L12] that this intersection complex is clean, and that therefore is supported on $\Sigma$, and is equal to $\iota_{\mathbf{L}} \otimes \mathcal{S}[\operatorname{dim} \Sigma]$ there.

We may now form the induced character sheaf (cf. [L85, (8.1.2), p. 237]), referred to as $K$ in loc. cit., but which we shall also write $\operatorname{Ind}_{\mathbf{L}}^{\mathbf{G}}\left(\left(\iota_{\mathbf{L}} \otimes \mathcal{S}\right)[\operatorname{dim} \Sigma]\right)$. Lusztig has shown [L84b, 3.4] that $\operatorname{End}\left(\operatorname{Ind}_{\mathbf{L}}^{\mathbf{G}}\left(\left(\iota_{\mathbf{L}} \otimes\right.\right.\right.$ $\mathcal{S})[\operatorname{dim} \Sigma])) \simeq \mathcal{A}$, a finite dimensional $\overline{\mathbb{Q}}_{\ell^{-}}$algebra, isomorphic to a twisted group algebra of $W_{\mathbf{G}}\left(\mathbf{L}, \iota_{\mathbf{L}}, \mathcal{S}\right)$, the subgroup of the relative Weyl group $W_{\mathbf{G}}(\mathbf{L})=N_{\mathbf{G}}(\mathbf{L}) / \mathbf{L}$ which fixes the Kummer system $\mathcal{S}$ on $\mathbf{L}$, as well as the cuspidal pair $\iota_{\mathbf{L}}$ described above.

It follows that

$$
K=\operatorname{Ind}_{\mathbf{L}}^{\mathbf{G}}\left(\left(\iota_{\mathbf{L}} \otimes \mathcal{S}\right)[\operatorname{dim} \Sigma]\right) \simeq \oplus_{E \in \operatorname{Irr}(\mathcal{A})} A_{\iota_{\mathbf{L}}, \mathcal{S}, E} \otimes V_{E},
$$

where $\operatorname{Irr}(\mathcal{A})$ denotes the set of irreducible characters of $\mathcal{A}$, and for each $E \in \operatorname{Irr}(\mathcal{A}), A_{\iota_{\mathrm{L}}, \mathcal{S}, E}$ is an irreducible character sheaf on $\mathbf{G}$ and $V_{E}$ is a $\overline{\mathbb{Q}}_{\ell^{-}}$ representation of $\mathcal{A}$, with character $E$. Generally, the data $\mathbf{L}, \iota_{\mathbf{L}}$ and $\mathcal{S}$ will be fixed, and when there is no risk of confusion, we write $A_{E}$ for $A_{\iota_{\mathrm{L}}, \mathcal{S}, E}$.

Remark 3.2. We shall denote character sheaves by $A_{E}$, but will sometimes need to refer to the associated cuspidal data. In that case, we write $\left(\mathbf{L}, \iota_{\mathbf{L}}, \mathcal{S}\right)(E)=\left(\mathbf{L}(E), \iota_{\mathbf{L}}(E), \mathcal{S}(E)\right)$ for the relevant data. 


\subsection{Characteristic functions}

Now suppose that $\mathbf{L}$ and $\Sigma$ above are $F$-stable. The stabiliser $\left\{w F \in W_{\mathbf{G}}(\mathbf{L}) F \mid F^{*} \dot{w}^{*}\left(\iota_{\mathbf{L}} \otimes \mathcal{S}\right) \simeq\left(\iota_{\mathbf{L}} \otimes \mathcal{S}\right)\right\}$ is a subcoset $W_{\mathbf{G}}(\mathbf{L}, \mathcal{S}) w_{1} F \leq$ $W_{\mathbf{G}}(\mathbf{L}) F$. Now for $E$ such that there is an isomorphism $F^{*} A_{E} \stackrel{\stackrel{\phi}{\longrightarrow}}{\longrightarrow} A_{E}$, we have an associated characteristic function $\chi_{E, \phi}: \mathbf{G}^{F} \rightarrow \overline{\mathbb{Q}}_{\ell}$, defined as an alternating sum on the cohomology of $A_{E}$ in the usual way.

In the above situation, there is an isomorphism $\phi_{0}: F^{*} \dot{w}_{1}^{*} K \rightarrow K$, which permutes the canonical decomposition (3.1). Note that $F^{*} \dot{w}_{1}^{*}$ acts on this decomposition as $F^{*} \otimes F^{*} \dot{w}_{1}^{*}$. Thus for each $E \in \operatorname{Irr}(\mathcal{A})$ such that $F^{*} \dot{w}_{1}^{*} E \simeq$ $E$, $\phi_{0}$ restricts to $\phi_{E} \otimes \sigma_{E} \in \operatorname{End}\left(A_{E} \otimes V_{E}\right)$, and hence for each choice of $\sigma_{E}$ defines $\phi_{E}: F^{*} A_{E} \rightarrow A_{E}$. The associated characteristic function $\chi_{E, \phi_{E}}$ : $\mathbf{G}^{F} \rightarrow \overline{\mathbb{Q}}_{\ell}$ is what we refer to as a Lusztig function. The various Lusztig functions, suitably normalised, form an orthonormal basis of the space of class functions on $\mathbf{G}^{F}$. For further details, see [L85, 10.4, 10.6].

\subsection{Families, Lusztig series and the wave front set}

The set $\hat{\mathbf{G}}$ of character sheaves on $\mathbf{G}$ is partitioned into families: $\hat{\mathbf{G}}=$ $\amalg_{\mathcal{L}, c} \hat{\mathbf{G}}_{\mathcal{L}, c}$, where $\mathcal{L}$ is a Kummer system on a fixed maximal torus $\mathbf{T}$ of $\mathbf{G}$ and $c$ is a family in the group $W_{\mathbf{G}}(\mathcal{L})$ (see [L85, 16.7 and 17.4] for this partition and the definition of two-sided cells and families in this group), two such pairs being considered equivalent if they are conjugate under the Weyl group. Now it is shown in [L92b, Thm. 10.7] that

Proposition 3.3. Given a family $(\mathcal{L}, c)$ there is a unique unipotent class $C$, called the unipotent support of the family, such that for any character sheaf $A_{E} \in \hat{\mathbf{G}}_{\mathcal{L}, c}$, its stalk at $g=s u \in \mathbf{G}$ (Jordan decomposition) is zero if $\operatorname{dim}(u) \geq \operatorname{dim} C$ and $u \notin C$, and there exists $u \in C$ such that the stalk at $u$ is nonzero for some $A_{E} \in \hat{\mathbf{G}}_{\mathcal{L}, c}$.

The last statement is a consequence of [L92b, (g) page 172].

The Lusztig functions correspond to the $F$-stable sheaves in the $F$-stable families $(\mathcal{L}, c)$, and we may therefore identify the set of Lusztig functions with $\hat{\mathbf{G}}^{F}$ and consequently have a partition of this set as

$$
\hat{\mathbf{G}}^{F}=\amalg_{(\mathcal{L}, c): F(\mathcal{L}, c)=(\mathcal{L}, c)} \hat{\mathbf{G}}_{\mathcal{L}, c}^{F},
$$

and the spaces spanned by the distinct $\hat{\mathbf{G}}_{\mathcal{L}, c}^{F}$ are orthogonal. 
Correspondingly, there is a partition ( $c f$. [L92b, 11.1]) of the irreducible characters, whose parts we call again families:

$$
\operatorname{Irr} \mathbf{G}^{F}=\amalg_{(\mathcal{L}, c): F(\mathcal{L}, c)=(\mathcal{L}, c)} \operatorname{Irr} \mathbf{G}^{F}{ }_{(\mathcal{L}, c)} .
$$

These partitions, both of characters and of character sheaves, are defined by the blocks of the matrix $\left\langle\rho, \chi_{E, \phi_{E}}\right\rangle_{\mathbf{G}^{F}}$. This allows us to identify families of irreducible characters and families of character sheaves. Thus specifically, $\left\langle\rho, \chi_{E, \phi_{E}}\right\rangle_{\mathbf{G}^{F}} \neq 0$ only if both $\rho$ and $A_{E}$ belong to the family parameterised by the pair $(\mathcal{L}, c)$.

Definition 3.4. The (Lusztig) series of an irreducible character $\rho$ (resp. character sheaf $A_{E}$ ) is said to be the Kummer local system $\mathcal{L}$ on $\mathbf{T}$ if $\rho \in$ $\operatorname{Irr}\left(\mathbf{G}^{F}\right)_{\mathcal{L}, c}\left(\right.$ resp. $\left.A_{E} \in \hat{\mathbf{G}}_{\mathcal{L}, c}\right)$.

We say that $\rho$ (resp. $A_{E}$ or $\chi_{E, \phi_{E}}$ ) is unipotent if its series $\mathcal{L}$ is equal to the trivial sheaf $\overline{\mathbb{Q}}_{\ell}$.

Note that the Kummer system $\mathcal{L}$ on the maximal torus $\mathbf{T}$ of $\mathbf{G}$ corresponds to a semisimple element $s \in \mathbf{G}^{*}$, the group dual to $\mathbf{G}$. We may therefore write $(s, c)$ for the family $(\mathcal{L}, c)$, and for $s \in \mathbf{G}^{* F}$ we denote by $\mathcal{E}\left(\mathbf{G}^{F}, s\right)$ the Lusztig series $\cup_{c} \operatorname{Irr}\left(\mathbf{G}^{F}\right)_{s, c}$ of irreducible characters. The unipotent characters correspond to $s=1$, or equivalently $\mathcal{L}=\overline{\mathbb{Q}}_{\ell}$.

Let $\chi$ be an irreducible character of $\mathbf{G}^{F}$. Lusztig has shown [L92b, 11.2] that the following definitions make sense.

Definition 3.5. (i) The wave front set $\mathrm{wf}(\chi)$ of $\chi$ is the largest unipotent class $C$ such that $\chi$ is a component of the corresponding generalised Gelfand-Graev representation $\Gamma_{u}$ for some $u \in C^{F}$.

(ii) The unipotent support of $\chi$ is the largest unipotent class $C$ such that $\chi$ has a non zero value on some element with unipotent part in $C^{F}$.

In the above, "largest" means that for any class of higher dimension, or of same dimension as $C$ but different from $C$, the multiplicity (resp. the value) is 0 .

Remark 3.6. Proposition 2.12 applies in particular to any irreducible character $\chi$ whose unipotent support is the class $(v)$. For in that case, if $(u)>(v)$, then by Scholium 2.3 $D \Gamma_{u}$ is supported on unipotent classes $\left(u^{\prime}\right)$ with $\left(u^{\prime}\right) \geq(u)>(v)$, and $\chi$ vanishes on such classes, whence $\left\langle\chi, D \Gamma_{u}\right\rangle_{\mathbf{G}^{F}}=0$. 
Given a family $c$ of $W_{\mathbf{G}}(\mathcal{L})$, let $c \otimes \varepsilon$ denote the family defined by the property that $\psi \in c$ if and only if $\psi \otimes \varepsilon \in c \otimes \varepsilon$, where $\varepsilon$ is the alternating character of $W$ restricted to $W_{\mathbf{G}}(\mathcal{L})$. With this notation, Lusztig [L92b] has proved the following properties of the sets defined above.

Proposition 3.7. Let $\chi \in \operatorname{Irr}\left(\mathbf{G}^{F}\right)_{(\mathcal{L}, c)}$ be an irreducible character and let $C$ be its unipotent support and $C^{\prime}$ its wave front set. Then

(i) There exists $u \in C^{F}$ such that $\chi(u) \neq 0$.

(ii) $C$ is the wave front set of the Alvis-Curtis dual of $\chi$.

(iii) $C^{\prime}$ is the unipotent support of $(\mathcal{L}, c \otimes \varepsilon)$.

Proof. (i) is [L92b, Thm. 11.2(v)], (ii) is [L92b, Thm. 11.2(iv)]. (iii) is [L92b, Thm. 11.2(i) and (iii)], taking into account [L92b, 11.1].

One would expect that in the situation of Proposition 3.7. $C$ is the unipotent support of $(\mathcal{L}, c)$; that is, any character $\chi \in \operatorname{Irr}\left(\mathbf{G}^{F}\right)_{(\mathcal{L}, c)}$ has the same unipotent support, and this support is the unipotent support of $(\mathcal{L}, c)$. We shall not require this in the current work. It would be a consequence of the assertion that if $\chi \in \operatorname{Irr}\left(\mathbf{G}^{F}\right)_{(\mathcal{L}, c)}$ then the Alvis-Curtis dual $D \chi$ of $\chi$ is in $\operatorname{Irr}\left(\mathbf{G}^{F}\right)_{(\mathcal{L}, c \otimes \varepsilon)}$. If $\mathbf{G}$ has connected center this last fact follows from $\mathbf{L} 84$, $6.14]$.

In view of the properties 3.7, we make the following definition.

Definition 3.8. The wave front set of a family $(\mathcal{L}, c)$ is the unipotent support of $(\mathcal{L}, c \otimes \varepsilon)$; equivalently, it is the common wave front set of all characters in $\operatorname{Irr}\left(\mathbf{G}^{F}\right)_{\mathcal{L}, c}$. If $A_{E} \in \hat{\mathbf{G}}_{\mathcal{L}, c}$ we shall denote by $\mathrm{wf}(E)$ the wave front set of its family and $\operatorname{supp}(E)$ the unipotent support of its family.

The following explicit description of the map between families and their wave front set may be found in $[\mathrm{L} 92 \mathrm{~b}, 10.5,10.6]$. Let $(\mathcal{L}, c)$ be a family, and let $s \in \mathbf{G}^{*}$ be a semi-simple element corresponding to $\mathcal{L}$. Let us write $W^{\prime}(s) \simeq W_{\mathbf{G}}(\mathcal{L})$ for the Weyl group of the not necessarily connected group $C_{\mathbf{G}^{*}}(s)$. The Weyl group of the identity component is denoted by $W(s)$. Let $E$ be a special representation of $c \otimes \varepsilon$; by definition, its restriction to $W(s)$ is a sum of special representations; let $E_{1}$ be one of these and consider $j_{W(s)}^{W}\left(E_{1}\right)$. This is an irreducible representation of $W$, and its Springer correspondent is supported by a unipotent class which is independent of the choice of $E_{1}$ and is the unipotent support of $(\mathcal{L}, c \otimes \varepsilon)$ i.e. the wave front set of $(\mathcal{L}, c)$. 
Conversely, given a unipotent class $C$, we obtain Lusztig families having $C$ as wave front set as follows. First note, that for a sufficiently large power $F^{m}$ of $F$, since the generalised Gelfand-Graev characters of $G^{F^{m}}$ form a basis of the space of unipotently supported class functions, each unipotent class is the wave front set of some irreducible character, hence of some family $(\mathcal{L}, c)$. Accordingly, if $E^{\prime}$ is the Springer correspondent of the pair $\left(C, \overline{\mathbb{Q}}_{\ell}\right)$, then using the above description of the wave front set, we see that there is always an $s$ such that the $j$-restriction of $E^{\prime}$ to $W(s)$ is a non zero special representation $E$ of $W(s)$. For any such $s$ this $E$ defines a family of $W(s)$, and hence families $c^{\prime}$ of $W^{\prime}(s)$. The class $C$ is now the wave front set of any character in one of the families $\left(s, c^{\prime} \otimes \varepsilon\right)$.

Definition 3.9. We say that a character is regular (resp. subregular) if its wave front set is the regular (resp. a subregular) class.

Given the above description of the wave front set, we may characterise the subregular characters in a Lusztig series as follows.

Lemma 3.10. Let $\mathbf{G}$ be simply connected. For any s the subregular characters in the Lusztig series $\mathcal{E}\left(\mathbf{G}^{F}, s\right)$ are precisely the characters in the family $\left(s, c^{\prime}\right)$, where $c^{\prime}$ is a family of $W^{\prime}(s)$ whose restriction to $W(s)$ contains one of the characters $\varepsilon \otimes r_{i}$ where $r_{i}$ is the reflection representation of the $i$-th irreducible component of $W(s)$.

Proof. A case-by-case check (see for example table [DLM3, 4.1]) shows that when $W$ is irreducible, the trivial local system on the unique subregular class $C$ corresponds to the reflection representation, a special character of $W$ (for this last fact the reflection representation is alone in its family in simply laced types by [L79]; for the other types one may use the description in [L79b]). It follows that in general the components of the reflection representation correspond to the trivial system on the various subregular classes.

But the restriction to $W(s)$ of the reflection representation is the sum of the $r_{i}$ and $(\operatorname{rank}(W)-\operatorname{rank}(W(s)))$ times the identity; hence the $j$ restriction of the reflection representation is the sum of the $r_{i}$, and the result follows.

\subsection{Multiplicities in generalised Gelfand-Graev representations}

Let $\mathcal{I}$ be the principal block, attached to the cuspidal pair $\left(\mathbf{T}, \iota_{0}\right)$, where $\iota_{0}$ is the pair $\left(1, \overline{\mathbb{Q}}_{\ell}\right)$. The aim of this subsection is to compute some multiplicities of characters in $\Gamma_{\iota}$ for $\iota \in \mathcal{I}$. 
For $s \in\left(\mathbf{G}^{*}\right)^{F}$, denote by $w_{1} F$ the type of a maximally split torus of the centraliser of $s$. For a $W(s)$-class function $f$ on $W(s) w_{1} F$ we define a class function in $\mathcal{E}\left(\mathbf{G}^{F}, s\right)$ by $R_{f}:=\frac{1}{|W(s)|} \sum_{y \in W(s) w_{1} F} f(y) R_{\mathbf{T}_{y}}^{\mathbf{G}}(\theta)$, where $R_{\mathbf{T}_{y}}^{\mathbf{G}}(\theta)$ is the Deligne-Lusztig character where for $y \in W F$ we denote by $\mathbf{T}_{y}$ an $F$-stable torus such that $\left(\mathbf{T}_{y}, F\right)$ is $\mathbf{G}$-conjugate to $(\mathbf{T}, y)$ and $\theta \in \operatorname{Irr}\left(\mathbf{T}_{y}^{F}\right)$ corresponds to $s \in \mathbf{G}^{* F}$.

Proposition 3.11. For $\iota$ in the principal block $\mathcal{I}$, the projection of $\Gamma_{\iota}$ onto $\mathcal{E}\left(\mathbf{G}^{F}, s\right)$ is, with notation as above

$$
a_{\iota} \sum_{\gamma \in \mathcal{I}^{F}} \widetilde{P}_{\iota, \gamma}^{*} R_{\operatorname{Res}_{W(s) w_{1} F}^{W F}\left(\widetilde{\varphi_{\gamma}} \otimes \widetilde{\varepsilon}\right)},
$$

where $\varphi_{\gamma} \in \operatorname{Irr}(W)^{F}$ is the Springer correspondent of $\gamma$ and $\widetilde{\varphi_{\gamma}}$ and $\tilde{\varepsilon}$ are preferred extensions to $W F$, in particular $\tilde{\varepsilon}(v F)=\varepsilon(v)$ (see [L85, 17.2]).

Proof. Lusztig's formulas [L92b, $\$ 7.5$ (b) and p.176 (b), proof of 11.2], suitably modified for the non-split case may be applied to give [DLM3, Proposition 6.1] which, applied to the principal block yields

$$
\Gamma_{\iota}=a_{\iota} Q^{\mathbf{G}}\left(\tilde{\varepsilon} \mathcal{Z}_{\mathbf{T}} \widetilde{Q_{\iota}^{*}}\right),
$$

where $\mathcal{Z}_{\mathbf{T}}$ is the function on $W F$ given by $\mathcal{Z}_{\mathbf{T}}(y)=y \mapsto\left|\mathbf{T}_{y}^{F}\right|, \widetilde{Q_{\iota}}(y)=$ $\sum_{\gamma \in \mathcal{I}^{F}} \widetilde{\varphi_{\gamma}}(y) \widetilde{P}_{\iota, \gamma}$, and $Q^{\mathbf{G}}$ is the map which sends the function $f$ to $\frac{1}{|W|} \sum_{y \in W F} f(y) R_{\mathbf{T}_{y}}^{\mathbf{G}}\left(\mathcal{X}_{\left(1, \overline{\mathbb{Q}}_{\ell}\right), w}\right)$. But $\mathcal{X}_{\left(1, \overline{\mathbb{Q}}_{\ell}\right), w}=\left|\mathbf{T}_{y}^{F}\right|^{-1} \sum_{\theta \in \operatorname{Irr}\left(\mathbf{T}_{y}^{F}\right)} \theta$ is the characteristic function of the identity (see [DLM3, Definition 3.1(iii) and Proposition 3.2]). It follows that

$$
\Gamma_{\iota}=\frac{a_{\iota}}{|W|} \sum_{\gamma \in \mathcal{I}^{F}} \widetilde{P}_{\iota, \gamma}^{*} \sum_{y \in W F, \theta \in \operatorname{Irr}\left(\mathbf{T}_{y}^{F}\right)}\left(\widetilde{\varphi_{\gamma}} \otimes \tilde{\varepsilon}\right)(y) R_{\mathbf{T}_{y}}^{\mathbf{G}}(\theta) .
$$

Identifying characters with elements of the dual group, the inner sum can be written

$$
\sum_{y \in W F, t \in \mathbf{T}^{* y}}\left(\widetilde{\varphi_{\gamma}} \otimes \tilde{\varepsilon}\right)(y) R_{\mathbf{T}_{y}}^{\mathbf{G}}(t) .
$$

Let $\chi$ be an irreducible character in the series $\mathcal{E}\left(\mathbf{G}^{F}, s\right)$. Then in the expansion of the inner product $\left\langle\Gamma_{\iota}, \chi\right\rangle_{\mathbf{G}^{F}}$, only the summands where $(y, t)$ is $W$ conjugate to some $\left(y_{0}, s\right)$ could be non zero, and if $(y, t)=\left(y_{0}, s\right)$ we have $y_{0} \in W(s) w_{1} F$. Further, a term $\left(\widetilde{\varphi_{\gamma}} \otimes \tilde{\varepsilon}\right)(y) R_{\mathbf{T}_{y}}^{\mathbf{G}}(t)$ depends only on the $W$ conjugacy class of $(y, t)$. Since the number of pairs $(y, t)$ conjugate to $\left(y_{0}, s\right)$ 
is $\frac{|W|}{\left|C_{W(s)}\left(y_{0}\right)\right|}$ and the number of pairs $\left(y^{\prime}, s\right)$ conjugate to $\left(y_{0}, s\right)$ is $\frac{|W(s)|}{\left|C_{W(s)}\left(y_{0}\right)\right|}$, we get

$$
\left\langle\Gamma_{\iota}, \chi\right\rangle_{\mathbf{G}^{F}}=\frac{a_{\iota}}{|W(s)|} \sum_{\gamma \in \mathcal{I}^{F}, y^{\prime} \in W(s) w_{1} F}\left(\widetilde{\varphi}_{\gamma} \otimes \widetilde{\varepsilon}\right)(y) \widetilde{P}_{\iota, \gamma}^{*}\left\langle R_{\mathbf{T}_{y^{\prime}}}^{\mathbf{G}}(s), \chi\right\rangle_{\mathbf{G}^{F}},
$$

whence the proposition follows, given the definition of $R_{f}$.

Proposition 3.13. Let $\chi \in \mathcal{E}\left(\mathbf{G}^{F}, s\right)$ have wave front set $C$, and let $\iota$ be in the principal block. Then

$$
\left\langle R_{\operatorname{Res}_{W(s) w_{1} F}^{W F}(\widetilde{\varphi} \otimes \widetilde{\varepsilon})}, \chi\right\rangle_{\mathbf{G}^{F}}=0
$$

unless $\operatorname{dim} \operatorname{supp} \iota<\operatorname{dim} C$ or $\operatorname{supp} \iota=C$.

Proof. Assume $\left\langle R_{\operatorname{Res}_{W(s) w_{1} F}^{W F}\left(\widetilde{\varphi_{\iota}} \otimes \widetilde{\varepsilon}\right)}, \chi\right\rangle_{\mathbf{G}^{F}} \neq 0$ and let $(s, c)$ be the family of $\chi$, which is also (see section 3.3) the family of some component $\psi$ of $\operatorname{Res}_{W(s) w_{1} F}^{W F}\left(\widetilde{\varphi}_{\iota} \otimes \widetilde{\varepsilon}\right)$. For a unipotent class $C$, let us denote by $\beta_{C}$ the dimension of the variety of Borel subgroups containing an element of $C$. By [L92b, Corollary 10.9 (i)] applied with $E^{\prime}=\psi \otimes \varepsilon$, we have $\beta_{\operatorname{supp} \iota} \geq a(c \otimes \varepsilon)$. We also know by part (h) of the proof of [L92b, Thm. 10.7] that $a(c \otimes \varepsilon)=\beta_{C}$ where $C$ is the class $C(s, c \otimes \varepsilon)$ as in [L92b, 10.5], that is (see the beginning of section 3 $C$ is the wave front set of all characters in the family $c$. So if $\left\langle R_{\operatorname{Res}_{W(s) w_{1} F}^{W F}\left(\widetilde{\varphi_{\iota}} \otimes \widetilde{\varepsilon}\right)}, \chi\right\rangle_{\mathbf{G}^{F}} \neq 0$ then $\operatorname{dim} \operatorname{supp} \iota \leq \operatorname{dim} C$. Moreover by [L92b, Corollary 10.9(ii)], if equality pertains, then $\operatorname{supp} \iota=C$.

Using the above propositions we now prove

Corollary 3.14. The assumptions and notation being as in the above proposition, let $\iota$ be a pair in the principal block.

(i) If $\chi \in \mathcal{E}\left(\mathbf{G}^{F}, s\right)$ has wave front set $\operatorname{supp} \iota$ then

$$
\left\langle\Gamma_{\iota}, \chi\right\rangle_{\mathbf{G}^{F}}=a_{\iota}\left\langle R_{\operatorname{Res}_{W(s) w_{1} F}^{W F}\left(\widetilde{\varphi_{\iota}} \otimes \widetilde{\varepsilon}\right)}, \chi\right\rangle_{\mathbf{G}^{F}}
$$

(ii) If ८ has subregular support, $\chi \in \mathcal{E}\left(\mathbf{G}^{F}, s\right)$ is regular, and $\mathbf{G}$ has connected centre, then

$$
\left\langle\Gamma_{\iota}, \chi\right\rangle_{\mathbf{G}^{F}}= \begin{cases}a_{\iota}\left(q+\left\langle\operatorname{Res}_{W(s) w_{1} F}^{W F} \widetilde{\varphi}_{\iota}, \mathrm{Id}\right\rangle_{W(s) w_{1} F}\right) & \text { if } \iota \text { is standard } \\ a_{\iota}\left\langle\operatorname{Res}_{W(s) w_{1} F}^{W F} \widetilde{\varphi}_{\iota}, \mathrm{Id}\right\rangle_{W(s) w_{1} F} & \text { otherwise. }\end{cases}
$$


When $\chi$ is subregular, in the notation of Lemma 3.10, we can write (i) above as

$$
a_{\iota} \sum_{E}\left\langle\operatorname{Res}_{W(s) w_{1} F}^{W F}\left(\widetilde{\varphi}_{\iota} \otimes \widetilde{\varepsilon}\right), \tilde{E}\right\rangle_{W(s) w_{1} F}\left\langle R_{\tilde{E}}, \chi\right\rangle_{\mathbf{G}^{F}}
$$

where $E$ runs over the $w_{1} F$-invariant characters of $W(s)$ which are in the family $c^{\prime}$.

Proof. We first prove (i). If $\operatorname{supp} \iota$ is the wave front set of $\chi$, by Proposition 3.13 we know that the $\gamma$ in 3.12 giving rise to a nonzero scalar product with $\chi$ must have support smaller than $\operatorname{supp} \iota$. On the other hand $P_{\iota, \gamma}^{*}$ is 0 unless the support of $\gamma$ is greater than that of $\iota$; then since the diagonal blocks of $P_{\iota, \gamma}^{*}$ are the identity, only the term in the formula survives.

We now prove (ii). If $\iota$ has subregular support then (see proof of Corollary 2.7 apart from $\widetilde{P}_{\iota, \iota}^{*}=1$ the only other non-zero $\widetilde{P}_{\iota, \gamma}^{*}$ occurs when $\iota$ is standard and $\gamma$ is the trivial local system on the regular class, in which case $\varphi_{\gamma}=\mathrm{Id}$ and $\widetilde{P}_{\iota, \gamma}^{*}=q$. Moreover, since the centre of $\mathbf{G}$ is connected, the regular character of the series $s$ is equal to $R_{\tilde{\varepsilon}}$. This gives the result.

In case the centre of $\mathbf{G}$ is connected, any regular character is orthogonal to $\mathcal{C}_{\mathcal{I}}\left(\mathbf{G}^{F}\right)$ for all non principal $\mathcal{I}$ since the only local system on the regular class is the trivial one. This leads to the following result.

Proposition 3.15. Assume that the centre of $\mathbf{G}$ is connected. Let $s$ be a semisimple element of $\left(\mathbf{G}^{*}\right)^{F}$ and let $\chi_{s}$ denote the regular character of $\mathcal{E}\left(\mathbf{G}^{F}, s\right)$. Then for any subregular rational unipotent element $u$ we have

$$
\left\langle\Gamma_{u}, \chi_{s}\right\rangle_{\mathbf{G}^{F}}=q \sum_{\gamma} \overline{\mathcal{Y}_{\gamma}(u)}+\sum_{\iota} \overline{\mathcal{Y}_{\iota}(u)}\left\langle\operatorname{Res}_{W(s) w_{1} F}^{W F} \widetilde{\varphi}_{\iota}, \operatorname{Id}\right\rangle_{W(s) w_{1} F},
$$

where the first sum is over the standard pairs with subregular support in the principal block and the second sum is over all pairs in the principal block with subregular support.

Proof. By the orthogonality relations for the $\mathcal{Y}_{\iota}$ (see for example DLM3, 4.2]) we have

$$
\Gamma_{u}=|A(u)|^{-1} \sum_{\iota} \overline{\mathcal{Y}_{\iota}(u)} \Gamma_{\iota},
$$

whence the result, by Corollary 3.14(ii) and the above remark. 
In the particular case where there is only one pair in the principal block with subregular support, the above formula becomes

$$
\left\langle\Gamma_{u}, \chi_{s}\right\rangle_{\mathbf{G}^{F}}=q+\left\langle\operatorname{Res}_{W(s) w_{1} F}^{W F} \widetilde{r}, \operatorname{Id}\right\rangle_{W(s) w_{1} F},
$$

where $r$ is the reflection representation of $W$.

\section{Restriction of character sheaves to the unipotent set.}

In DLM3 the interrelationships among various bases of the space $\mathcal{C}_{\text {uni }}\left(\mathbf{G}^{F}\right)$ of unipotently supported functions is discussed, and Lusztig induction and restriction were described in these terms. In this section we discuss the restriction of the characteristic function of an arbitrary Frobenius-stable character sheaf to the unipotent set of $\mathbf{G}^{F}$, and in particular give a formula for its inner product with the generalised Gelfand-Graev character $\Gamma_{u}$. Such characteristic functions are referred to as Lusztig functions (see $\$ 3.2$ ).

We begin by describing the restriction of the Lusztig functions $\chi_{E, \phi_{E}}$ to the unipotent set $\mathbf{G}_{\text {uni }}^{F}$. It is known by [L86, pp. 151] that when the local system $\iota_{\overline{\mathbf{L}}}$ as in 33.1 has unipotent support, then $W_{\mathbf{G}}\left(\mathbf{L}, \iota_{\mathbf{L}}\right)=W_{\mathbf{G}}(\mathbf{L})$, and that the implied cocycle is trivial, so that $\mathcal{A} \simeq \overline{\mathbb{Q}}_{\ell} W_{\mathbf{G}}(\mathbf{L}, \mathcal{S})$ and the Lusztig functions in the corresponding block can be indexed by characters of $W_{\mathbf{G}}(\mathbf{L}, \mathcal{S})$.

Theorem 4.1. Let $\chi_{E, \phi_{E}}$ be a Lusztig function with associated cuspidal data $\left(\mathbf{L}, \iota_{\mathbf{L}}, \mathcal{S}\right)$ as in 3.1 and 3 3.2. Then

(i) If $\iota_{\overline{\mathbf{L}}}$ has support which is not a unipotent class, then the restriction $\left.\chi_{E, \phi_{E}}\right|_{\mathbf{G}_{u n i}^{F}}$ is zero.

(ii) If $\iota_{\overline{\mathbf{L}}}$ has unipotent support, then $E \in \operatorname{Irr} \mathcal{A}=\operatorname{Irr} W_{\mathbf{G}}(\mathbf{L}, \mathcal{S})$. In this case, we have

$$
\left.\chi_{E, \phi_{E}}\right|_{\mathbf{G}_{u n i}^{F}}=(-1)^{\operatorname{dim} \operatorname{supp} \iota_{\mathbf{L}}} Q^{\mathbf{G}}\left(\operatorname{Ind}_{W_{\mathbf{G}}(\mathbf{L}, \mathcal{S}) \cdot w_{1} F}^{W_{\mathbf{L}}(\mathbf{L}) \cdot F}(\overline{\tilde{E}})\right)
$$

where $\tilde{E}$ is a suitable extension of $E$ and $Q^{\mathbf{G}}$ is the map from $\mathcal{C}\left(W_{\mathbf{G}}(\mathbf{L}) . F\right)$ to $\mathcal{C}_{\mathcal{I}(E)}\left(\mathbf{G}^{F}\right)$ defined by $Q^{\mathbf{G}}\left(\widetilde{\varphi}_{\kappa}\right)=\widetilde{\mathcal{X}}_{\kappa}$ (see [DLM3, Def. 3.1]). Here $\mathcal{I}(E)$ is the block corresponding to the unipotently supported cuspidal local system $\iota_{\overline{\mathbf{L}}}$.

Proof. The first part is in [L85, Th. 8.5]. We therefore assume that $\iota_{\overline{\mathbf{L}}}$ has unipotent support, so that $\mathcal{A}=\overline{\mathbb{Q}}_{\ell} W_{\mathbf{G}}(\mathbf{L}, \mathcal{S})$. Then for any element 
$w \in W_{\mathbf{G}}(\mathbf{L}, \mathcal{S})$, we have an isomorphism $\phi_{0, w}^{*}: F^{*}\left(w w_{1}\right)^{*} K \rightarrow K$, and, for each $E$ such that $F^{*} \dot{w}_{1}^{*} E \simeq E$, an associated characteristic function $\chi_{E, \phi_{E}, w}$. Moreover those $E \in \operatorname{Irr} W_{\mathbf{G}}(\mathbf{L}, \mathcal{S})$ which are fixed by $F^{*} \dot{w}_{1}^{*}$ are precisely the same as those fixed by $F^{*}\left(\dot{w} \dot{w}_{1}\right)^{*}$. Following Lusztig [L85, §10.4], for the characteristic functions, for each $w \in W_{\mathbf{G}}(\mathbf{L}, \mathcal{S})$, we have

$$
\chi_{K, \phi_{0, w}}=\sum_{E: w_{1}^{*} F^{*} E=E} \tilde{E}\left(w w_{1} F\right) \chi_{E, \phi_{E}},
$$

where $\tilde{E}$ is a suitable extension of $E$ to $W_{\mathbf{G}}(\mathbf{L}, \mathcal{S}) \cdot w_{1} F$. Inverting this relation using the orthogonality of characters of cosets, we obtain as in [L85, formula $(10.4 .5)]$

$$
\chi_{E, \phi_{E}}=\left|W_{\mathbf{G}}(\mathbf{L}, \mathcal{S})\right|^{-1} \sum_{w \in W_{\mathbf{G}}(\mathbf{L}, \mathcal{S})} \overline{\tilde{E}\left(w w_{1} F\right)} \chi_{K, \phi_{0, w}} .
$$

But again by [L85, Th. 8.5] (or [DLM3, Prop. 3.2]) the restriction of $\chi_{K, \phi_{0, w}}$ to the unipotent set is $(-1)^{\operatorname{dim} \operatorname{supp} \iota_{\mathbf{L}}} Q_{w w_{1}}^{\mathbf{G}}$ (the sign comes from the fact that the perverse sheaf is shifted from the intersection cohomology complex by $\left.\operatorname{dim} \operatorname{supp} \iota_{\mathbf{L}}\right)$, and by [DLM3, Def. 3.1(iii)] we have $Q_{w w_{1}}^{\mathbf{G}}=Q^{\mathbf{G}}\left(\gamma_{w w_{1}}\right)$, where $\gamma_{v}$ denotes the normalised characteristic function of the class of $v F$ in $W_{\mathbf{G}}(\mathbf{L}) \cdot F$. It follows that

$$
\operatorname{Res}_{\mathbf{G}_{\mathrm{uni}}^{F}}^{\mathbf{G}^{F}}\left(\chi_{E, \phi_{E}}\right)=(-1)^{\operatorname{dimsupp} \iota_{\mathbf{L}}}\left|W_{\mathbf{G}}(\mathbf{L}, \mathcal{S})\right|^{-1} \sum_{v \in W_{\mathbf{G}}(\mathbf{L}, \mathcal{S}) \cdot w w_{1} F} \overline{\tilde{E}(v)} Q^{\mathbf{G}}\left(\gamma_{w w_{1}}\right) .
$$

But by Frobenius' formula for induced characters,

$$
\left|W_{\mathbf{G}}(\mathbf{L}, \mathcal{S})\right|^{-1} \sum_{v \in W_{\mathbf{G}}(\mathbf{L}, \mathcal{S}) \cdot w w_{1} F} \overline{\tilde{E}(v)} \gamma_{w w_{1}}=\operatorname{Ind}_{W_{\mathbf{G}}(\mathbf{L}, \mathcal{S}) \cdot w_{1} F}^{W_{\mathbf{G}}(\mathbf{L}) \cdot F}(\overline{\tilde{E}}),
$$

and the result follows.

Remark 4.3. Theorem 4.1 shows that given a Lusztig function $\chi_{E, \phi_{E}}$ with non-zero restriction to the unipotent set, its restriction lies in $\mathcal{C}_{\mathcal{I}(E)}$ for a well defined block $\mathcal{I}(E)$, which corresponds to the cuspidal local system $\iota_{\overline{\mathbf{L}}}(E)$, which has unipotent support.

\subsection{Wave front set and character sheaves}

In order to better understand the restriction to the unipotent set of a Lusztig function, we shall need the following information concerning the Lusztig 
functions which have non-trivial inner product with a given irreducible character.

Definition 4.4. With notation as in Theorem 4.1, we define $n_{\kappa}(E)$ by

$$
\operatorname{Ind}_{W_{\mathbf{G}}(\mathbf{L}, \mathcal{S}) \cdot w_{1} F}^{W_{\mathbf{G}}(\mathbf{L}) \cdot F}(\overline{\tilde{E}})=(-1)^{\operatorname{dimsupp} \iota_{\mathbf{L}}} \sum_{\kappa \in \mathcal{I}(E)} n_{\kappa}(E) \tilde{\varphi}_{\kappa} .
$$

Note that $n_{\kappa}(E)$ depends on the extension $\tilde{E}$ which itself depends on the chosen isomorphism $\phi_{E}$.

Remark 4.5. (i) For any $F$-stable character sheaf $A_{E}$, the scalars $n_{\kappa}(E)$ are defined by 4.4 , which implicitly involves the triple $\left(\mathbf{L}(E), \iota_{\mathbf{L}}(E), \mathcal{S}(E)\right)$ as described in Remark 3.2, and assumes that $\iota_{\overline{\mathbf{L}}}(E)$ has unipotent support, as is the case when the restriction of $\chi_{E, \phi_{E}}$ to the unipotent set of $\mathbf{G}^{F}$ is non-zero by Theorem 4.1(i). Whenever we use the notation $n_{\kappa}(E)$ we shall take this as understood.

(ii) The $n_{\kappa}$ are algebraic integers. They will figure prominently in the rest of this work.

The following key result generalises Proposition 3.13, which deals only with the principal block. Recall that in [DLM3, 3.6] we defined an involution $\iota \mapsto \hat{\iota}$ on a block $\mathcal{I}$ and a sign $\varepsilon_{\iota}$ on $\mathcal{I}$ by $\widetilde{\varphi}_{\iota} \otimes \widetilde{\varepsilon}=\varepsilon_{\iota} \widetilde{\varphi}_{\hat{\iota}}$ where $\widetilde{\varepsilon}$ is defined on $W_{\mathbf{G}}(\mathbf{L}) F$ by $\widetilde{\varepsilon}(w F)=\varepsilon(w)$. We have $\varepsilon_{\iota}=1$ when $\mathbf{G}$ is split (that is, $F$ acts trivially on $W$ ).

Lemma 4.6. Let $A_{E}$ be a character sheaf in $\hat{\mathbf{G}}_{\mathcal{L}, c}^{F}$, and suppose that $\chi_{E, \phi_{E}}$ has non-zero restriction to the unipotent set. Let $C$ be the wave front set of $(\mathcal{L}, c)$ and $C^{\prime}$ its unipotent support. If $n_{\kappa}(E) \neq 0$, then

(i) either the support of $\kappa$ is $C^{\prime}$, or it has dimension smaller than $\operatorname{dim}\left(C^{\prime}\right)$.

(ii) either the support of $\hat{\kappa}$ is $C$, or it has dimension smaller than $\operatorname{dim}(C)$.

Proof. By Theorem 4.1 and Definition 4.4 we have

$$
\operatorname{Res}_{\mathbf{G}_{\mathrm{uni}}^{F}}^{\mathbf{G}^{F}} \chi_{E, \phi_{E}}=\sum_{\kappa \in \mathcal{I}(E)} n_{\kappa}(E) \widetilde{\mathcal{X}}_{\kappa}
$$

The assertion (i) is now immediate from the definition of the support, since the transition matrix between the bases $\mathcal{X}_{\iota}$ and $\mathcal{Y}_{\iota}$ is unitriangular. 
We now apply the duality functor $D$ to 4.7 , bearing in mind that duality commutes with restriction to the unipotent set and [DLM3, 3.14 (ii)], obtaining

$$
\operatorname{Res}_{\mathbf{G}_{\mathrm{uni}}^{F}}^{\mathbf{G}^{F}} D\left(\chi_{E, \phi_{E}}\right)=\sum_{\kappa \in \mathcal{I}(E)} n_{\kappa}(E) \eta_{\mathbf{L}(E)} \varepsilon_{\kappa} \widetilde{\mathcal{X}}_{\hat{\kappa}}
$$

Now $\chi_{E, \phi_{E}}$ is a linear combination of characters in the same family:

$$
\chi_{E, \phi_{E}}=\sum_{\rho \in \operatorname{Irr}\left(\mathbf{G}^{F}\right)_{\mathcal{L}, c}} m_{\rho} \rho
$$

whence

$$
D\left(\chi_{E, \phi_{E}}\right)=\sum_{\rho \in \operatorname{Irr}\left(\mathbf{G}^{F}\right)_{\mathcal{L}, c}} m_{\rho} D(\rho),
$$

where all the $D(\rho)$ have unipotent support $C$, since all the $\rho$ have wave front set $C$. Again using the fact that the transition matrix between the bases $\mathcal{X}_{\iota}$ and $\mathcal{Y}_{\iota}$ is triangular, this means that the restriction to the unipotent set of $D\left(\chi_{E, \phi_{E}}\right)$ is a linear combination of $\mathcal{X}_{\iota}$ with the support of $\iota$ either equal to $C$ or having smaller dimension. Comparing with 4.8 , we obtain (ii).

\subsection{Inner product with Generalised Gelfand-Graev characters}

We begin with the following general expression for the Mellin transform $\Gamma_{\iota}$ in terms of Lusztig functions. Note that since the Lusztig functions form an orthonormal basis of $\mathcal{C}\left(\mathbf{G}^{F}\right)$, it suffices for this purpose to compute the inner product of $\Gamma_{\iota}$ with an arbitrary Lusztig function $\chi_{E, \phi_{E}}$. Moreover such an inner product is evidently zero unless the restriction of $\chi_{E, \phi_{E}}$ to $\mathbf{G}_{\mathrm{uni}}^{F}$ is nonzero, in which case $A_{E}$ determines a unipotently supported cuspidal local system $\iota_{\overline{\mathbf{L}}}(E)$, a corresponding block $\mathcal{I}(E)$ and a unipotent class $\mathrm{wf}(E)$, viz. its wave front set (see Definition 3.8).

The following result is related to Proposition 3.11 .

Theorem 4.9. Let $\iota \in \mathcal{I}^{F}$, a block with cuspidal datum $\left(\mathbf{L}, \iota_{\mathbf{L}}\right)$ and suppose $A_{E} \in \hat{G}^{F}$. In terms of the integers $n_{\kappa}(E)$ of Definition 4.4. we have

$$
\left\langle\widetilde{\Gamma}_{\iota}, \chi_{E, \phi_{E}}\right\rangle_{\mathbf{G}^{F}}=\left\{\begin{array}{l}
0 \text { if } \operatorname{Res}_{\mathbf{G}_{u n i}^{F}}^{\mathbf{G}^{F}}\left(\chi_{E, \phi_{E}}\right)=0 \text { or } \mathcal{I}(E) \neq \mathcal{I} \\
\sum_{\substack{\kappa \in \mathcal{I}^{F}, \kappa \geq \text { and } \\
\operatorname{dim}(\sup (\kappa))<\operatorname{dim}(\mathbf{w f}(E)) \\
\text { or } \operatorname{supp}(\kappa)=\operatorname{wf}(E)}} n_{\hat{\kappa}}(E) \varepsilon_{\kappa} \widetilde{P}_{\iota \kappa}^{*} \text { otherwise. }
\end{array}\right.
$$


Similarly

$$
\left\langle D\left(\widetilde{\Gamma}_{\iota}\right), \chi_{E, \phi_{E}}\right\rangle_{\mathbf{G}^{F}}=\left\{\begin{array}{l}
0 \text { if } \operatorname{Res}_{\mathbf{G}_{u n i}^{F}}^{\mathbf{G}^{F}}\left(\chi_{E, \phi_{E}}\right)=0 \text { or } \mathcal{I}(E) \neq \mathcal{I} \\
\eta_{\mathbf{L}} \sum_{\substack{\kappa \in \mathcal{I}^{F}, \kappa \geq \iota \text { and } \\
\operatorname{dim}(\operatorname{supp}(\kappa))<\operatorname{dim}(\operatorname{supp}(E)) \\
\text { or } \operatorname{supp}(\kappa)=\operatorname{supp}(E)}} n_{\kappa}(E) \widetilde{P}_{\iota \kappa}^{*} \text { otherwise. }
\end{array}\right.
$$

Proof. Using equation 4.7) we get

$$
\left\langle\widetilde{\Gamma}_{\iota}, \chi_{E, \phi_{E}}\right\rangle_{\mathbf{G}^{F}}=\left\langle\widetilde{\Gamma}_{\iota}, \operatorname{Res}_{\mathbf{G}_{\mathrm{uni}}^{F}}^{\mathbf{G}^{F}}\left(\chi_{E, \phi_{E}}\right)\right\rangle_{\mathbf{G}^{F}}=\sum_{\kappa \in \mathcal{I}(E)} n_{\kappa}(E)\left\langle\widetilde{\Gamma}_{\iota}, \widetilde{\mathcal{X}}_{\kappa}\right\rangle_{\mathbf{G}^{F}}
$$

In the proof of [DLM3, 6.2] one finds the equation $\eta_{\mathbf{L}} \varepsilon_{\kappa}\left\langle\Gamma_{\iota}, \tilde{\mathcal{X}}_{\hat{\kappa}}\right\rangle_{\mathbf{G}^{F}}=$ $\eta_{\mathbf{L}} a_{\iota} \zeta_{\mathcal{I}}^{-1} \tilde{P}_{\iota, \kappa}^{*}$ which by the definition of $\widetilde{\Gamma}_{\iota}$ can be written $\left\langle\widetilde{\Gamma}_{\iota}, \tilde{\mathcal{X}}_{\hat{\kappa}}\right\rangle_{\mathbf{G}^{F}}=$ $\varepsilon_{\kappa} \tilde{P}_{\iota, \kappa}^{*}$.

Changing the variable from $\kappa$ to $\hat{\kappa}$ in the equation above and substituting, we obtain 4.10 , the condition on $\operatorname{supp}(\kappa)$ comes from Lemma 4.6(ii) and the condition $\kappa \geq \iota$ from the fact that $P_{\iota, \gamma}$ is upper triangular.

For formula 4.11, since duality commutes with restriction to the unipotent set, the function $D\left(\widetilde{\Gamma}_{\iota}\right)$ is also unipotently supported so we have the same equation with $\widetilde{\Gamma}_{\iota}$ replaced by $D\left(\widetilde{\Gamma}_{\iota}\right)$. We then use [DLM3, 3.14(ii)] which states that $D\left(\tilde{\mathcal{X}}_{\kappa}\right)=\eta_{\mathbf{L}} \varepsilon_{\kappa} \tilde{\mathcal{X}}_{\hat{\kappa}}$ and proceed in the same way, using 4.6 (i) instead of 4.6 (ii).

Corollary 4.12. With notation as in Theorem 4.9. if $\operatorname{dim}(\operatorname{supp}(\iota)) \geq$ $\operatorname{dim}(w f(E))$ then

$$
\left\langle\widetilde{\Gamma}_{\iota}, \chi_{E, \phi_{E}}\right\rangle_{\mathbf{G}^{F}}=\left\{\begin{array}{l}
n_{\hat{\iota}}(E) \varepsilon_{\iota} \text { if } \operatorname{supp}(\iota)=\mathrm{wf}(E) \text { and } \mathcal{I}(E)=\mathcal{I}, \\
0 \text { otherwise. }
\end{array}\right.
$$

and if $\operatorname{dim}(\operatorname{supp}(\iota)) \geq \operatorname{dim}(\operatorname{supp}(E))$ then

$$
\left\langle D\left(\widetilde{\Gamma}_{\iota}\right), \chi_{E, \phi_{E}}\right\rangle_{\mathbf{G}^{F}}=\left\{\begin{array}{l}
\eta_{\mathbf{L}} n_{\iota}(E) \text { if } \operatorname{supp}(\iota)=\operatorname{supp}(E) \text { and } \mathcal{I}(E)=\mathcal{I}, \\
0 \text { otherwise. }
\end{array}\right.
$$

Proof. The condition on the first case of equation 4.13 (resp. equation 4.14) is such that there is only one summand satisfying the conditions in the sum on the right side of (4.10) (resp. of (4.11)), and it corresponds to $\kappa=\iota$. In the second case, there is no $\kappa$ satisfying those conditions.

We next prove the "Mellin transform" of the statement in Theorem 4.9 . 
Corollary 4.15. Let $A_{E}$ be a character sheaf such that $\operatorname{Res}_{\mathbf{G}_{u n i}^{F}}^{\mathbf{G}^{F}} \chi_{E, \phi_{E}} \neq 0$. Let $C$ be a unipotent class in $\mathbf{G}$ and $u \in C^{F}$. Then, again in terms of the integers $n_{\kappa}(E)$ of Definition 4.4, we have

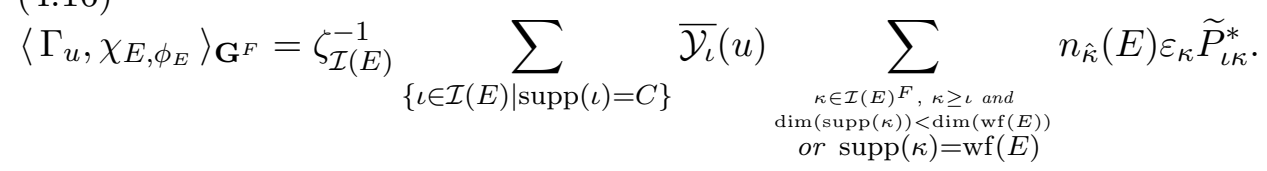

Proof. Using 3.16 and the definition $\Gamma_{\iota}=|A(u)| \zeta_{\mathcal{I}}^{-1} \widetilde{\Gamma}_{\iota}$, we have

$$
\Gamma_{u}=\sum_{\iota \in \mathcal{P}^{F}} \zeta_{\mathcal{I}(\iota)}^{-1} \overline{\mathcal{Y}_{\iota}(u)} \widetilde{\Gamma}_{\iota}
$$

where $\mathcal{I}(\iota)$ is the block to which $\iota$ belongs. The corollary now follows immediately from Theorem 4.9 .

The next corollary gives the multiplicity of a Lusztig function in a generalised Gelfand-Graev character corresponding to a unipotent element of its wave front set.

Corollary 4.17. Maintain the notation of Corollary 4.15 and assume that $u \in \operatorname{wf}(E)^{F}$. Then

$$
\left\langle\Gamma_{u}, \chi_{E, \phi_{E}}\right\rangle_{\mathbf{G}^{F}}=\zeta_{\mathcal{I}}^{-1} \sum_{\{\iota \in \mathcal{I}(E) \mid \operatorname{supp}(\iota)=\operatorname{wf}(E)\}} n_{\hat{\iota}}(E) \varepsilon_{\iota} \overline{\mathcal{Y}}_{\iota}(u),
$$

Proof. We use the relation (4.16) to compute the left side. Since $u \in \mathrm{wf}(E)$, it follows that in the outer sum all the $\iota$ have support $\mathrm{wf}(E)$, and hence in the inner sum of (4.16), there is just one summand, viz. the term corresponding to $\kappa=\iota$. The result follows.

The next result is a consequence of Corollary 4.12 .

Corollary 4.18. Given a unipotent class $C$, let $\mathcal{C}_{\mathrm{wf}(C)}\left(\mathbf{G}^{F}\right)$ (resp. $\mathcal{C}_{\operatorname{supp}(C)}\left(\mathbf{G}^{F}\right)$ ) be the space of class functions on $\mathbf{G}^{F}$ which has basis the irreducible characters $\rho \in \operatorname{Irr}\left(\mathbf{G}^{F}\right)_{(\mathcal{L}, c)}$ where $C$ is the wavefront of $(\mathcal{L}, c)$ (resp the support of $(\mathcal{L}, c)$ ). Write $\operatorname{Proj}_{\mathrm{wf}(C)}\left(\right.$ resp. $\left.\operatorname{Proj}_{\operatorname{supp}(C)}\right)$ for the projection onto this space with respect to the complement spanned by the other irreducible characters. If $\kappa \in \mathcal{I}^{F}$ (a block with cuspidal datum $\left(\mathbf{L}, \iota_{\mathbf{L}}\right)$ ) has 
support $C$, then we have

$$
\operatorname{Proj}_{\mathrm{wf}(C)} \widetilde{\Gamma}_{\kappa}=\varepsilon_{\kappa} \sum_{\substack{\left\{A_{E} \in(\hat{G}) F \mid \mathcal{F}(E)=\mathcal{I} \\ \text { and } \mathrm{wf}(E)=C\right\}}} n_{\hat{\kappa}}(E) \chi_{E, \phi_{E}} .
$$

and

$$
\operatorname{Proj}_{\operatorname{supp}(C)} D\left(\widetilde{\Gamma}_{\kappa}\right)=\eta_{\mathbf{L}} \sum_{\substack{\left\{A_{E} \in(\hat{G}) F(I(E)=\mathcal{I} \\ \text { and } \operatorname{supp}(E)=C\}\right.}} n_{\kappa}(E) \chi_{E, \phi_{E}} .
$$

Proof. Observe first that since the Lusztig functions form an orthonormal basis of the space of class functions on $\mathbf{G}^{F}$, we have

$$
\operatorname{Proj}_{\mathrm{wf}(C)} \widetilde{\Gamma}_{\kappa}=\sum_{\{E \mid \mathrm{wf}(E)=C\}}\left\langle\widetilde{\Gamma}_{\kappa}, \chi_{E, \phi_{E}}\right\rangle_{\mathbf{G}^{F}} \chi_{E, \phi_{E}} .
$$

Now, since we have $\operatorname{supp}(\kappa)=\operatorname{wf}(E)$ for the $E$ in the sum, we can apply 4.13 and we get the stated value for $\operatorname{Proj}_{\mathrm{wf}(C)} \widetilde{\Gamma}_{\kappa}$. 4.13

The proof for $\operatorname{Proj}_{\operatorname{supp}(C)} D\left(\widetilde{\Gamma}_{\kappa}\right)$ proceeds similarly, using 4.14 instead of

\section{Lusztig series and unipotent characters}

Recall (Definition 3.4) the definition of the Lusztig series of an irreducible character or character sheaf. We shall require the following two statements concerning the series to which a character sheaf belongs. This series is defined as above by a Kummer local system $\mathcal{T}$ on a maximal torus $\mathbf{T}$ of $\mathbf{G}$ [L85, $2.10]$.

Lemma 5.1. (i) Suppose $\left(\mathbf{L}, \iota_{\mathbf{L}}, \mathcal{S}\right)$ is a triple as in $\$ 3.1$. Let $\mathcal{T}_{\mathcal{S}}$ be the lift to $\mathbf{T}$ of $\mathcal{S}$ and let $\mathcal{T}_{0}$ be the Lusztig series of the cuspidal triple $\left(\mathbf{L}, \iota_{\mathbf{L}}, \overline{\mathbb{Q}}_{\ell}\right)$. Then the Lusztig series of any character sheaf $A_{E}$ induced from $\left(\mathbf{L}, \iota_{\mathbf{L}}, \mathcal{S}\right)$ is $\mathcal{T}_{0} \otimes \mathcal{T}_{\mathcal{S}}$.

(ii) Suppose $A_{E}$ and $A_{E^{\prime}}$ are respectively character sheaves induced from $\left(\mathbf{L}, \iota_{\mathbf{L}}, \mathcal{S}\right)$ and $\left(\mathbf{L}, \iota_{\mathbf{L}}, \mathcal{S}^{\prime}\right)$. If $A_{E}$ and $A_{E^{\prime}}$ are in the same Lusztig series, then $\mathcal{S}$ and $\mathcal{S}^{\prime}$ are conjugate under $W_{\mathbf{G}}(\mathbf{L})$.

Proof. The statement (i) may be found in [L85, 17.9]. Given this, the hypothesis of (ii) implies that for some $w \in W_{\mathbf{G}}(\mathbf{L}), \mathcal{T}_{0} \otimes \mathcal{T}_{\mathcal{S}^{\prime}}=\left(\mathcal{T}_{0} \otimes \mathcal{T}_{\mathcal{S}}\right)^{w}$. But $\mathcal{T}_{0}$ is stable under $W_{\mathbf{G}}(\mathbf{L})$ since $W_{\mathbf{G}}\left(\mathbf{L}, \iota_{\mathbf{L}}\right)=W_{\mathbf{G}}(\mathbf{L})$ (see beginning of section 
40. It follows that $\mathcal{T}_{0} \otimes \mathcal{T}_{\mathcal{S}^{\prime}}=\mathcal{T}_{0} \otimes\left(\mathcal{T}_{\mathcal{S}}\right)^{w}$. As $\mathcal{T}_{0}$ is a Kummer system, we may multiply by its inverse, obtaining the result.

Lemma 5.2. Maintain the notation of Lemma 5.1; in particular, $\mathcal{T}_{0}$ is the Lusztig series of the cuspidal triple $\left(\mathbf{L}, \iota_{\mathbf{L}}, \overline{\mathbb{Q}}_{\ell}\right)$.

(i) If $\iota_{\overline{\mathbf{L}}}$ is unipotently supported, then either $\mathcal{T}_{0}$ is trivial, or else it corresponds to an element $t_{0} \in \mathbf{L}^{*}$ whose centraliser is not the whole of $\mathbf{L}^{*}$.

(ii) If $\iota_{\overline{\mathbf{L}}}$ is unipotently supported and $A_{E}$ is a unipotent character sheaf induced from $\left(\mathbf{L}, \iota_{\mathbf{L}}, \mathcal{S}\right)$, then both $\mathcal{T}_{0}$ and $\mathcal{S}$ are trivial Kummer systems.

Proof. The first statement is verified by a case by case check, using the classification of cuspidal character sheaves given in the Appendix below. Note that the restriction that the characteristic be good is necessary for the truth of the assertion (i).

To see (ii), note that by Lemma 5.1 (i), the Lusztig series of $A_{E}$ is a Kummer system which corresponds to the element $t_{0} s \in \mathbf{T}^{*}$, the torus dual to $\mathbf{T}$, where $t_{0}$ is as given, and $s$ corresponds to $\mathcal{S}$. Since $\mathcal{S}$ is trivial on $[\mathbf{L}, \mathbf{L}], s$ is centralised by $\mathbf{L}^{*}$. Now $A_{E}$ is unipotent precisely when $t_{0} s=1$; but by (i), if $t_{0} \neq 1$, then $t_{0}$ is not centralised by $\mathbf{L}^{*}$, whence $t_{0} s \neq 1$. Thus if $A_{E}$ is unipotent, $t_{0}=s=1$.

The restriction to the unipotent set is simpler for unipotent character sheaves:

Lemma 5.3. Let $A_{E}$ be a unipotent character sheaf induced from $\left(\mathbf{L}, \iota_{\mathbf{L}}, \mathcal{S}\right)$. If $\phi_{E}$ is such that $\tilde{E}$ of 4.1 (ii) is the preferred extension, then we have

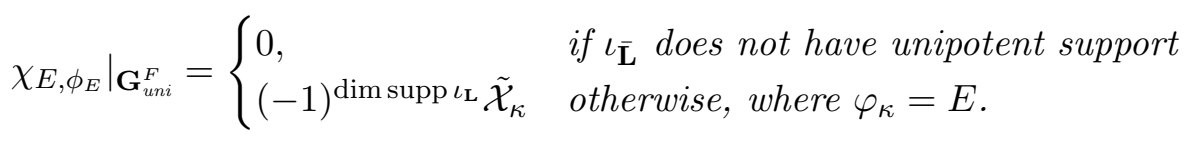

Proof. The fact that the restriction is 0 if $\iota_{\overline{\mathbf{L}}}$ does not have unipotent support results from 4.1(i).

If we now assume that $\iota_{\overline{\mathbf{L}}}$ is unipotently supported then by Lemma 5.2(ii) both $\mathcal{S}$ and $\mathcal{T}_{0}$ of Lemma 5.1(i) are trivial.

Further, by [L84b, 9.2], since $\iota_{\overline{\mathbf{L}}}$ is unipotently supported, then $W_{\mathbf{G}}\left(\mathbf{L}, \iota_{\mathbf{L}}\right)=W_{\mathbf{G}}(\mathbf{L})$. Formula 4.2 reduces thus to the statement of the lemma, taking in account that the preferred extension takes rational values. 
Denote by Proj $\mathrm{uni}_{\text {un }}$ the projection onto the space spanned by the unipotent characters of $\mathbf{G}^{F}$ with respect to the complement spanned by the other characters, that is, the orthogonal projection onto $\mathcal{E}\left(\mathbf{G}^{F}, 1\right)$.

The next result shows that a certain projection of $\widetilde{\Gamma}_{\iota}$ is precisely a Lusztig function. We say that a block $\mathcal{I}$ is in the unipotent Lusztig series if its cuspidal datum is $\left(\mathbf{L}, \iota_{\mathbf{L}}\right)$ and the Lusztig series of a character sheaf with cuspidal datum $\left(\mathbf{L}, \iota_{\mathbf{L}}, \overline{\mathbb{Q}}_{\ell}\right)$ is the unipotent series $\left(\mathcal{T}_{0}=\overline{\mathbb{Q}}_{\ell}\right.$ in the language of Lemma 5.1).

Proposition 5.4. Let $\kappa \in \mathcal{I}$ be a pair with support the unipotent class $C$, and assume $\left(\mathbf{L}, \iota_{\mathbf{L}}\right)$ is the cuspidal data of $\mathcal{I}$. Then

$\operatorname{Proj}_{u n i} \operatorname{Proj}_{\mathrm{wf}(C)}\left(\widetilde{\Gamma}_{\kappa}\right)= \begin{cases}(-1)^{\operatorname{dim} \operatorname{supp} \iota_{\mathbf{L}}} \varepsilon_{\kappa} \chi_{E, \phi_{E}} & \begin{array}{l}\text { if } \mathcal{I} \text { is in the unipotent } \\ \text { Lusztig series, } \\ \text { otherwise }\end{array}\end{cases}$

where $A_{E}$ is the character sheaf with cuspidal data $\left(\mathbf{L}, \iota_{\mathbf{L}}, \overline{\mathbb{Q}}_{\ell}\right)$ determined by

$$
E:=\varphi_{\hat{\kappa}} \in \operatorname{Irr}\left(W_{\mathbf{G}}\left(\mathbf{L}, \overline{\mathbb{Q}}_{\ell}\right)\right)=\operatorname{Irr}\left(W_{\mathbf{G}}(\mathbf{L})\right)
$$

Similarly, we have

$\operatorname{Proj}_{\text {uni }} \operatorname{Proj}_{\operatorname{supp}(C)}\left(D\left(\widetilde{\Gamma}_{\kappa}\right)\right)= \begin{cases}(-1)^{\operatorname{dimsupp} \iota_{\mathbf{L}}} \eta_{\mathbf{L}} \chi_{E, \phi_{E}} \\ \text { if } \mathcal{I} \text { is in the unipotent } \\ \text { Lusztig series, } \\ \text { otherwise }\end{cases}$

where $A_{E}$ is determined by $E:=\varphi_{\kappa} \in \operatorname{Irr}\left(W_{\mathbf{G}}(\mathbf{L})\right)$.

Proof. In the formula for $\operatorname{Proj}_{\mathrm{wf}(u)} \widetilde{\Gamma}_{\kappa}$ in Corollary 4.18 , for any $E$ in the right-hand side, $\operatorname{Proj}_{\text {uni }}\left(\chi_{E, \phi_{E}}\right)$ is either $\chi_{E, \phi_{E}}$ or zero, according as $A_{E}$ is unipotent or not. In the first case, by Lemma 5.3 there is just one $E$ for which $n_{\hat{\kappa}}(E) \neq 0$, namely $E=\varphi_{\hat{\kappa}}$, and for this $E$, we have $n_{\hat{\kappa}}(E)=(-1)^{\operatorname{dim} \operatorname{supp} \iota_{\mathrm{L}}}$. This completes the computation of $\operatorname{Proj}_{\text {uni }} \operatorname{Proj}_{\mathrm{wf}(C)}\left(\widetilde{\Gamma}_{\kappa}\right)$.

The computation of $\operatorname{Proj}_{\text {uni }} \operatorname{Proj}_{\operatorname{supp}(C)}\left(D\left(\widetilde{\Gamma}_{\kappa}\right)\right)$ proceeds similarly.

Corollary 5.5. Let $A_{E}$ be a unipotent character sheaf with cuspidal data $\left(\mathbf{L}, \iota_{\mathbf{L}}, \overline{\mathbb{Q}}_{\ell}\right)$ determined by $E:=\varphi_{\hat{\kappa}} \in \operatorname{Irr}\left(W_{\mathbf{G}}(\mathbf{L})\right)$. Then $D\left(\chi_{E, \phi_{E}}\right)=$ $\varepsilon_{\kappa} \eta_{\mathbf{L}} \chi_{E^{\prime}, \phi_{E^{\prime}}}$ where $A_{E^{\prime}}$ is the unipotent character sheaf with same cuspidal datum determined by $E^{\prime}:=\varphi_{\kappa} \in \operatorname{Irr}\left(W_{\mathbf{G}}(\mathbf{L})\right)$. 
Proof. The statement follows by comparing the dual of the first expression given in 5.4 with the second expression, given that the dual of a family of unipotent characters with wavefront $C$ is a family of unipotent characters with support $C$. We prove this latter fact.

By Proposition 3.7(iii), if $C$ is the wavefront of a family $(\mathcal{L}, c)$, then it is the support of the family $(\mathcal{L}, c \otimes \varepsilon)$. Since for $E \in \operatorname{Irr}(W)$ we have $D\left(R_{E}\right)=R_{E \otimes \varepsilon}$ (notation as in the second paragraph of subsection 3.4) and a unipotent character is in a family $\left(\overline{\mathbb{Q}}_{\ell}, c\right)$ if and only if it has non-zero multiplicity in $R_{E}$ for some $E$ in that family, it follows that the dual of the family $\left(\overline{\mathbb{Q}}_{\ell}, c\right)$ is the family $\left(\overline{\mathbb{Q}}_{\ell}, c \otimes \varepsilon\right)$.

Fix the datum $\left(\mathbf{L}, \iota_{\mathbf{L}}, \mathcal{S}\right)$ as described in $\$ 3.1$. Given an element $\theta=$ $\sum_{E \in \operatorname{Irr}\left(W_{\mathbf{G}}(\mathbf{L}, \mathcal{S})\right)} c_{E} E$ of the character ring of $W_{\mathbf{G}}(\mathbf{L}, \mathcal{S})$ over $\overline{\mathbb{Q}}_{\ell}$, define

$$
\chi_{\theta}:=\sum_{E \in \operatorname{Irr}\left(W_{\mathbf{G}}(\mathbf{L}, \mathcal{S})\right)} c_{E} \chi_{E, \phi_{E}} .
$$

We shall now generalise Corollary 3.14 to the case of characters not necessarily in the principal series.

Proposition 5.6. Let $\rho \in \operatorname{Irr}\left(\mathbf{G}^{F}\right)$ have wave front set $C$, and let $\kappa$ be a pair with support $C$ in the block $\mathcal{I}$, which has cuspidal datum $\left(\mathbf{L}, \iota_{\mathbf{L}}\right)$. Then

(i) All Lusztig functions $\chi_{E, \phi_{E}}$ satisfying $\left\langle\rho, \chi_{E, \phi_{E}}\right\rangle_{\mathbf{G}^{F}}\left\langle\widetilde{\Gamma}_{\kappa}, \chi_{E, \phi_{E}}\right\rangle_{\mathbf{G}^{F}} \neq$ 0 , arise from a single datum $\left(\mathbf{L}, \iota_{\mathbf{L}}, \mathcal{S}\right)$ as in 3.1 .

(ii) With $\left(\mathbf{L}, \iota_{\mathbf{L}}, \mathcal{S}\right)$ as in (i), we have

$$
\left\langle\widetilde{\Gamma}_{\kappa}, \rho\right\rangle_{\mathbf{G}^{F}}=\varepsilon_{\kappa}\left\langle\rho, \chi_{\operatorname{Res}_{W_{\mathbf{G}}(\mathbf{L}, \mathcal{S}) \cdot w_{1} F}^{W_{\mathbf{L}}(\mathbf{L}) \cdot F} \tilde{\varphi}_{\hat{\kappa}}}\right\rangle_{\mathbf{G}^{F}}
$$

Proof. We first prove (i). If $\left\langle\rho, \chi_{E, \phi_{E}}\right\rangle_{\mathbf{G}^{F}} \neq 0$ then $C=\operatorname{wf}(\rho)=\operatorname{wf}(E)$ and from Corollary 4.18(i) if further $\left\langle\bar{\Gamma}_{\kappa}, \chi_{E, \phi_{E}}\right\rangle_{\mathbf{G}^{F}} \neq 0$ then $\mathcal{I}(E)=\mathcal{I}$. Thus the part $\left(\mathbf{L}, \iota_{\mathbf{L}}\right)$ of the cuspidal datum for $A_{E}$ is determined by the block $\mathcal{I}$. Furthermore, $\left\langle\rho, \chi_{E, \phi_{E}}\right\rangle_{\mathbf{G}^{F}} \neq 0$ implies that the Lusztig series of $A_{E}$ is determined by, and equal to, that of $\rho$, so that by Lemma 5.1 the Kummer system $\mathcal{S}$ in the datum for $A_{E}$ is also determined by the stated conditions.

We now prove (ii). Assume that $\rho \in \operatorname{Irr}\left(\mathbf{G}^{F}\right)_{\mathcal{T}, c}$. Then $\rho$ may be expressed as

$$
\rho=\sum_{\chi_{E, \phi_{E}} \in \hat{\mathbf{G}}_{\mathcal{T}, c}^{F}}\left\langle\rho, \chi_{E, \phi_{E}}\right\rangle_{\mathbf{G}^{F}} \chi_{E, \phi_{E}} .
$$


It follows using Corollary 4.18 that

$$
\begin{aligned}
\left\langle\widetilde{\Gamma}_{\kappa}, \rho\right\rangle_{\mathbf{G}^{F}} & =\sum_{\chi_{E, \phi_{E}} \in \hat{\mathbf{G}}_{\mathcal{T}, c}^{F}}\left\langle\rho, \chi_{E, \phi_{E}}\right\rangle_{\mathbf{G}^{F}}\left\langle\widetilde{\Gamma}_{\kappa}, \chi_{E, \phi_{E}}\right\rangle_{\mathbf{G}^{F}} \\
& =\sum_{\chi_{E, \phi_{E}} \in \hat{\mathbf{G}}_{\mathcal{T}, c}^{F}}\left\langle\rho, \chi_{E, \phi_{E}}\right\rangle_{\mathbf{G}^{F}} n_{\hat{\kappa}}(E) \varepsilon_{\kappa}
\end{aligned}
$$

where by (i) the sum is restricted to those $E$ with a given cuspidal datum. Hence the above sum is over $E \in \operatorname{Irr}\left(W_{\mathbf{G}}(\mathbf{L}, \mathcal{S}) . w_{1} F\right)$, and by Frobenius reciprocity, $n_{\hat{\kappa}}(E)=\left\langle E \operatorname{Res}_{W_{\mathbf{G}}(\mathbf{L}, \mathcal{S}) \cdot w_{1} F}^{W_{\mathbf{G}}(\mathbf{\varphi}) \cdot F} \tilde{\varphi}_{\hat{\kappa}}\right\rangle_{W_{\mathbf{G}}(\mathbf{L}, \mathcal{S}) \cdot w_{1} F}$. Thus

$$
\left\langle\widetilde{\Gamma}_{\kappa}, \rho\right\rangle_{\mathbf{G}^{F}}=\varepsilon_{\kappa} \sum_{\chi_{E, \phi_{E}} \in \hat{\mathbf{G}}_{\mathcal{T}, c}^{F}}\left\langle\rho, \chi_{E, \phi_{E}}\right\rangle_{\mathbf{G}^{F}}\left\langle E, \operatorname{Res}_{W_{\mathbf{G}}(\mathbf{L}, \mathcal{S}) \cdot w_{1} F}^{W_{\mathbf{G}}(\mathbf{L}) \cdot F} \tilde{\varphi}_{\hat{\kappa}}\right\rangle_{W_{\mathbf{G}}(\mathbf{L}, \mathcal{S}) \cdot w_{1} F},
$$

and the proof is complete.

\section{Character values, projections, generalised Gelfand-Graev characters and families.}

\subsection{The setup}

In this section we are interested in the projection of Gelfand-Graev characters onto the space of unipotent characters and in the value of unipotent characters; since unipotent characters factor through the adjoint group, we assume $\mathbf{G}$ adjoint in this section.

We shall explore the following particular situation (cf. [L12b]). We consider a family $\mathcal{F}=(\mathcal{L}, c)$ of unipotent characters, so that $\mathcal{L}=\overline{\mathbb{Q}}_{\ell}$ and $c$ is a family in $W$ (see Definition 3.4 and beginning of section 3.3). The wave front set of such a family is called a special unipotent class $(u)$. We suppose that

(i) The group $\mathcal{G}$ attached to $\mathcal{F}$ (see below) is $A(u)$.

(ii) At most one of the local systems on $(u)$ is not in the principal block.

Note that

- In general the group $\mathcal{G}$ attached to $\mathcal{F}$ is a certain canonical quotient $\overline{A(u)}$ defined in [L84, after 13.1.2]; see also [L12b]. Thus condition (i) amounts to stipulating that $A(u)=\overline{A(u)}$. 
- Since $\mathbf{G}$ has connected centre, it is always possible to choose $u$ in its geometric class such that $F$ acts trivially on $A(u)$, see [T, Prop. 2.4]. We shall assume this property for the rest of this section, and hence label the rational classes contained in $(u)$ as $\left(u_{g}\right)$, where $g$ runs over a set of representatives of the conjugacy classes of $A(u)$.

Applying the discussion after Definition 3.8 to the particular case of the unipotent series, one sees that there is a unique family with wavefront $(u)$. Indeed, $(u)$ is defined by the fact that the Springer correspondent of the special character of the family is the local system $\left((u), \overline{\mathbb{Q}}_{\ell}\right)$.

Remark 6.2. The assumptions (6.1) are satisfied by all special unipotent classes in groups of type $G_{2}, F_{4}$ or $E_{8}$ such that $A(u)=\overline{A(u)}$ (that is, most special classes). In fact for these groups all local systems are in the principal block except one on the subregular class in type $G_{2}\left(A(u)=\mathrm{Sym}_{3}\right)$, on the class $F_{4}\left(a_{3}\right)$ in type $F_{4}\left(A(u)=\operatorname{Sym}_{4}\right)$, and on the class $E_{8}\left(a_{7}\right)$ (in the Bala-Carter notation) in type $E_{8}\left(A(u)=\mathrm{Sym}_{5}\right)$. Assumptions (6.1) are also satisfied in groups of type $E_{6}$ and $E_{7}$ where all local systems are in the principal block.

In the next subsection, we shall define Mellin transforms of the irreducible characters in $\mathcal{F}$, and show that in the cases covered by Remark 6.2 , the orthogonal projection of $\Gamma_{u_{g}}$ onto the family $\mathcal{F}$ is one of these Mellin transforms. A consequence of the proof will be the determination of all the values of the characters in $\mathcal{F}$ at unipotent classes in the cases of Remark 6.2 then, any unipotent irreducible character $\chi$ is either in $\mathcal{F}$ or else it is in the principal series in the sense of character sheaves. In the latter case, the values of $\chi$ on the unipotent set are given by Green functions, and may be determined algorithmically.

Thus a consequence of the results of this section is the determination of all the values of all unipotent irreducible characters of $\mathbf{G}^{F}$ at unipotent classes for $\mathbf{G}$ a group of type $G_{2}, F_{4}, E_{6},{ }^{2} E_{6}, E_{7}$ and $E_{8}$.

\subsection{Families, Fourier transform and almost characters}

We assume henceforth that $\mathbf{G}$ is quasi-simple (or equivalently that $W$ is irreducible). We give the basic facts concerning the Fourier transform of unipotent characters, following [DM1, $\S 3$, Ch. VII] and [DM2, 4.4]. With each family $\mathcal{F}$ of unipotent irreducible characters of $\mathbf{G}^{F}$, there is associated a finite group $\mathcal{G}$. The unipotent characters in the family $\mathcal{F}$ are parameterised 
by

$$
\mathcal{M}(\mathcal{G}):=\left\{(x, \chi) \mid x \in \mathcal{G}, \chi \in \operatorname{Irr}\left(C_{\mathcal{G}}(x)\right)\right\} / \mathcal{G},
$$

where the action of $\mathcal{G}$ is by simultaneous conjugation. Since the characters we consider are unipotent and we assume $W$ to be irreducible, it follows that $F$ fixes each $F$-stable family pointwise. Consequently, despite the fact that in general Lusztig considers a more complicated set than $\mathcal{M}(\mathcal{G})$ using an automorphism of $\mathcal{G}$ induced by $F$, we do not have to deal with this more general situation here.

To describe Lusztig's Fourier transform matrix we shall also require a $\operatorname{sign} \Delta_{(x, \chi)}$ defined by Lusztig; we will be more explicit about its value when needed. For the moment we note that

- If $\mathbf{G}$ is split, $\Delta_{(x, \chi)}=1$ except for the "exceptional" families in types $E_{7}$ and $E_{8}$ (those containing unipotent characters attached to irrational representations of the Hecke algebra).

- If $\mathbf{G}$ is nonsplit, $\Delta_{(x, \chi)}$ depends only on $\mathcal{F}$ (and not the particular $(x, \chi)$ considered); we will therefore denote it by $\Delta_{\mathcal{F}}$.

We write $\rho_{(x, \chi)}$ for the unipotent character parameterised by $(x, \chi)$. There are two other bases of the space $\mathcal{C}_{\mathcal{F}}$ spanned by the $\rho_{(x, \chi)}$ which play an important role.

Definition 6.3. (cf. [DM2, 4.4]). Let $\mathcal{M}^{\prime}(\mathcal{G}):=\{(x, y) \in \mathcal{G} \times \mathcal{G} \mid x y=$ $y x\} / \mathcal{G}$; note that $\mathcal{M}(\mathcal{G})$ and $\mathcal{M}^{\prime}(\mathcal{G})$ have the same cardinality.

(i) For a representative $(x, y)$ of $\mathcal{M}^{\prime}(\mathcal{G})$ (see above) define the Mellin transform

$$
\mu_{(x, y)}:=\sum_{\chi \in \operatorname{Irr}\left(C_{\mathcal{G}}(x)\right)} \chi(y) \rho_{(x, \chi)} .
$$

(ii) We define another basis $\left\{R_{(x, \chi)}\right\}_{(x, \chi) \in \mathcal{M}(\mathcal{G})}$ of $\mathcal{C}_{\mathcal{F}}$, the almost characters, by the property that $\mu_{(x, y)}=\Delta_{\mathcal{F}} \sum_{\chi \in \operatorname{Irr}\left(C_{\mathcal{G}}(x)\right)} \chi(x) R_{(y, \chi)}$, except in exceptional families of $E_{7}$ and $E_{8}$ where we have $\mathcal{G}=\mathbb{Z} / 2$ and if $\epsilon$ is the non-trivial character of $\mathcal{G}$ the above formula must be modified to read: $\mu_{(x, y)}=\epsilon(x) \sum_{\chi \in \operatorname{Irr}\left(C_{\mathcal{G}}(x)\right)} \bar{\chi}(x) R_{(y, \chi)}$ (this takes into account Lusztig's $\Delta_{(x, \chi)}$ in this case).

Definition (ii) above follows Lusztig [L84, 4.24.1 and 13.6]. Note that in loc. cit. 
- If $c$ is the family in the group $W$ corresponding to $\mathcal{F}$ (see the beginning of section 3.3), Lusztig parameterises the preferred extensions $\tilde{E}$ of characters of $c$ by some elements $x_{\tilde{E}} \in \mathcal{M}(\mathcal{G})$.

- When $\left(x^{\prime}, \chi^{\prime}\right)=x_{\tilde{E}}$ then $R_{\left(x^{\prime}, \chi^{\prime}\right)}$ is equal to the function $R_{\tilde{E}}$ of subsection 3.4

Clearly knowledge of the values of all the irreducible characters in $\mathcal{F}$ on a given class $C$ is equivalent to knowledge of the values on $C$ of the almost characters or of the Mellin transforms.

The following result is proved by Shoji (see [S1, Th. 5.7] and [S2, Th. 3.2 and Th. 4.1]). In rough language, it says that the Lusztig functions are, up to multiplication by a scalar which is a root of unity, equal to the almost characters of $\mathbf{G}^{F}$.

Proposition 6.4. As above, let $\mathcal{F}$ be a family with associated group $\mathcal{G}$. The Lusztig functions in $\mathcal{F}$ may, just as the irreducible characters, be labelled by pairs $(x, \phi) \in \mathcal{M}(\mathcal{G})$. Write $\chi_{(x, \phi)}$ for the Lusztig function corresponding to $(x, \phi) \in \mathcal{M}(\mathcal{G})$. Then for $(\mathbf{G}, F)$ with connected centre and $p$ sufficiently large the following is true. For each $(x, \phi) \in \mathcal{M}(\mathcal{G})$, there is an algebraic number $\zeta_{F}$ of absolute value 1 such that $\chi_{(x, \phi)}=\zeta_{F} R_{(x, \phi)}$.

We have written $\zeta_{F}$ to emphasise the dependency on $F$; this number of course a priori depends also on $(x, \phi)$. The condition "sufficiently large" for $p$ above is "almost good", which means good for exceptional groups, and no condition imposed for classical groups.

We shall now prove

Theorem 6.5. Maintain the assumption that is $\mathbf{G}$ quasi-simple. Suppose that we are in the setting of 6.1; in particular $\mathcal{F}$ is a family of unipotent characters of $\mathbf{G}^{F}$ and $(u)$ is its wave front set. Then:

(i) Any local system on $(u)$ is the (shifted) restriction of a character sheaf lying in the unipotent Lusztig series.

(ii) For any $g \in A(u)$, we have $\operatorname{Proj}_{\mathcal{F}}\left(\Gamma_{u_{g}}\right)=\Delta_{\mathcal{F}} D\left(\mu_{(g, 1)}\right)$ except for the exceptional families of $E_{7}$ and $E_{8}$ where we have $\operatorname{Proj}_{\mathcal{F}}\left(\Gamma_{u_{g}}\right)=$ $\epsilon(g) D\left(\mu_{(g, 1)}\right)$.

(iii) If there is a pair $\kappa \in \mathcal{I}$ with support $(u)$ where $\mathcal{I}$ is a non-principal block with cuspidal datum $\left(\mathbf{L}, \iota_{\mathbf{L}}\right)$, the root of unity $\zeta_{F}$ of Proposition 6.4 attached to $\kappa$ is equal to $\zeta_{\mathcal{I}}(-1)^{\operatorname{dim} \operatorname{supp} \iota_{\mathbf{L}}} \eta_{\mathbf{L}}$. 
Proof. In the bijection of [L12c, Th. 2.4 (b)] between unipotent character sheaves of $\mathcal{F}$ and $\mathcal{M}(\mathcal{G})$, the shifted restriction to the class $(u)$ of the character sheaf with label $(1, \chi)$ is the local system corresponding to $\chi$, so that all local systems on $(u)$ appear in this bijection, whence (i).

Now consider the case when all local systems on $(u)$ are in the principal block. Then by [S1, 2.18], for all $F$-invariant $E \in \operatorname{Irr}(W)$ we have $\chi_{E, \phi_{E}}=(-1)^{\mathrm{rank} \mathbf{G}} R_{\widetilde{E}}$ when $\sigma_{E}$ in subsection 3.2 has been chosen such that it defines the extension $\tilde{E}$, in particular $\zeta_{F}=(-1)^{\text {rank } \mathbf{G}}$. By Proposition 5.4, and the definition of $\hat{\kappa}$ above Lemma 4.6, if $E=\varphi_{\kappa}$ we have $\operatorname{Proj}_{\mathcal{F}}\left(\Gamma_{\kappa}\right)=a_{\kappa} \varepsilon_{\kappa} R_{\widetilde{E \otimes \varepsilon}}$ (here we use the fact that $\zeta_{\mathcal{I}}=1$ for the principal block and that $\operatorname{dim} \operatorname{supp} \iota_{\mathbf{L}}=\operatorname{rank} \mathbf{G}$ in Proposition 5.4). This can be written as $\operatorname{Proj}_{\mathcal{F}}\left(\Gamma_{\kappa}\right)=D\left(a_{\kappa} R_{\widetilde{E}}\right)$ since the Alvis-Curtis dual of $R_{\widetilde{E}}$ is $R_{\widetilde{E} \otimes \widetilde{\varepsilon}}=\varepsilon_{\kappa} R_{\widetilde{E \otimes \varepsilon}}($ see $[\mathrm{L} 84,6.8 .6])$. By [L12b, Cor. 0.5], if $\kappa=((u), \chi)$ then $\widetilde{E}$ is parameterised by $(1, \chi) \in \mathcal{M}(\mathcal{G})$, hence we have $\operatorname{Proj}_{\mathcal{F}}\left(\Gamma_{\kappa}\right)=D\left(a_{\kappa} R_{(1, \chi)}\right)$. By 3.16 we have

$$
\Gamma_{u_{g}}=a_{u}^{-1} \sum_{\psi \in \operatorname{Irr}(A(u))} \overline{\psi(g)} \Gamma_{((u), \psi)}
$$

Applying $\operatorname{Proj}_{\mathcal{F}}$ to both sides of this relation, and using the above we obtain

$$
\operatorname{Proj}_{\mathcal{F}}\left(\Gamma_{u_{g}}\right)=D\left(\sum_{\psi \in \operatorname{Irr}(A(u))} \overline{\psi(g)} R_{(1, \psi)}\right)
$$

By 6.3 (ii), the right side of 6.6 is equal to $\Delta_{\mathcal{F}} D\left(\mu_{(g, 1)}\right)$ except in the exceptional families of $E_{7}$ and $E_{8}$ where it is $\epsilon(g) D\left(\mu_{(g, 1)}\right)$.

In the case where there is a pair $\kappa=((u), \eta)$ not in the principal block but in a block $\mathcal{I}$ with cuspidal data $\left(\mathbf{L}, \iota_{\mathbf{L}}\right)$ then Proposition 5.4 . using Corollary 5.5 becomes $\operatorname{Proj}_{\mathcal{F}}\left(\Gamma_{\kappa}\right)=a_{\kappa} \zeta_{\mathcal{I}}^{-1}(-1)^{\operatorname{dimsupp} \iota_{\mathbf{L}}} \eta_{\mathbf{L}} D\left(\chi_{E, \phi_{E}}\right)$ where $A_{E}$ is determined by $E=\varphi_{\kappa}$. As explained in the beginning of the proof, the character sheaf $A_{E}$ has label $(1, \eta)$. Now applying Proposition 6.4 we get $\operatorname{Proj}_{\mathcal{F}}\left(\Gamma_{\kappa}\right)=a_{\kappa} \zeta_{\mathcal{I}}^{-1}(-1)^{\operatorname{dim} \operatorname{supp} \iota_{\mathbf{L}}} \eta_{\mathbf{L}} \zeta_{F} D\left(R_{(1, \eta)}\right)$, and writing $\zeta=\zeta_{\mathcal{I}}^{-1} \zeta_{F}(-1)^{\operatorname{dim} \operatorname{supp} \iota_{\mathbf{L}}} \eta_{\mathbf{L}}$, we obtain

$$
\begin{aligned}
\operatorname{Proj}_{\mathcal{F}}\left(\Gamma_{u_{g}}\right) & =D\left(\sum_{\substack{\psi \in \operatorname{Irr}(A(u)) \\
\psi \neq \eta \eta}} \overline{\psi(g)} R_{(1, \psi)}\right)+\zeta \overline{\eta(g)} D\left(R_{(1, \eta)}\right) \\
& =D\left(\sum_{\psi \in \operatorname{Irr}(A(u))} \psi\left(g^{-1}\right) R_{(1, \psi)}\right)+(\zeta-1) \eta\left(g^{-1}\right) D\left(R_{(1, \eta)}\right) \\
& =\Delta_{\mathcal{F}} D\left(\mu_{(g, 1)}\right)+(\zeta-1) \eta\left(g^{-1}\right) D\left(R_{(1, \eta)}\right)
\end{aligned}
$$


where $\mu$ is the Mellin transform as explained in the case where all local systems were in the principal block (here we use the fact that we are not in an exceptional family).

But the left hand side is a proper character of $\mathbf{G}^{F}$, and since $\mu_{(g, 1)}$ is a proper character and $R_{(1, \eta)}$ has rational coefficients in the basis of characters, it is immediate that $\zeta= \pm 1$. Case by case inspection shows that if there is a single pair with support $(u)$ not in the principal block, then $A(u) \in\left\{\operatorname{Sym}_{3}, \operatorname{Sym}_{4}, \operatorname{Sym}_{5}\right\}$ or $A(u) \simeq(\mathbb{Z} / 2 \mathbb{Z})^{k}$ with $k \geq 2$ (that is, since we assume $\mathbf{G}$ adjoint, $A(u) \not z \mathbb{Z} / 2 \mathbb{Z})$; indeed, for adjoint exceptional groups, both local systems are in the principal block when $\mathcal{G}=A(u)=\mathbb{Z} / 2 \mathbb{Z}$; for adjoint classical groups, if $\mathcal{G}=A(u)=\mathbb{Z} / 2 \mathbb{Z}$, one checks that the only pair $(x, \chi) \in \mathcal{M}(\mathcal{G})$ which parameterises a local system which is not in the principal block has $x \neq 1$ hence by [L12C, Th. 2.4 (b)] does not correspond to a local system on $(u)$.

In the non-abelian case $A(u) \in\left\{\mathrm{Sym}_{3}, \mathrm{Sym}_{4}, \mathrm{Sym}_{5}\right\}$, the formula in [DM1] for the Fourier transform shows that the coefficient of $\rho_{(y, \chi)}$ in $R_{(1, \eta)}$ is non-zero for any $\chi$ if $y$ is a 3 -cycle. If $\zeta=-1$, not all of the negative terms can be cancelled in the right side of the above equation.

In the abelian case $A(u)=(\mathbb{Z} / 2 \mathbb{Z})^{k}$ with $k>1$, if $\zeta=-1$ the coefficient of $\rho_{(y, \chi)}$ in $R_{(1, \eta)}$ has a denominator $2^{k-1}$ with $k$ as above. Hence in both cases we conclude that $\zeta=1$ and $\operatorname{Proj}_{\mathcal{F}}\left(\Gamma_{u_{g}}\right)=D\left(\mu_{(g, 1)}\right)$.

The statement (ii) of Theorem 6.5 proves implicitly that $\Delta_{\mathcal{F}} D\left(\mu_{(} g, 1\right)$ ) (resp. $\epsilon(g) D(\mu(g, 1)))$ is an actual character. We remark that this is also a consequence of the result [L84, 6.20] of Lusztig.

\subsection{Values of unipotent characters at unipotent elements, and concluding remarks}

In the three groups covered by Remark 6.2, as well as in groups of type $E_{6}$, ${ }^{2} E_{6}$ and $E_{7}$, all the families of unipotent characters other than one family $\mathcal{F}$ satisfying the assumptions of Theorem 6.5 are in the principal series, i.e. are of the form $\left(\overline{\mathbb{Q}}_{\ell}, c\right)$. Hence their values at unipotent elements are given by Green functions, which may be taken as known by Lusztig's algorithm.

Thus to determine all values of all unipotent characters in $\operatorname{Irr}\left(\mathbf{G}^{F}\right)$ at unipotent elements, it suffices to determine the values of the characters in $\mathcal{F}$. But by Proposition 6.4 for this it suffices to determine the root of unity $\zeta_{F}$, since the values of the Lusztig functions $\chi_{(x, \phi)}((x, \phi) \in \mathcal{M}(\mathcal{F}))$ are known. Hence in these cases, Theorem 6.5(ii) completes the determination of the values of unipotent characters at unipotent classes. The constant $\zeta_{F}$ was 
already determined by Lusztig [L86, 8.6] and implicitely by Kawanaka [K2, 4.2.2], using different methods which each yield different restrictions on the applicable values of $p$ and $q$.

We remark finally that empirically, the values of the Mellin transforms $\mu_{(x, y)}$ at unipotent classes appear to be "simpler" than the values of the irreducible characters, when expressed as polynomials in $q$. This remark is based on computations in the exceptional groups, and currently we have no theoretical justification for it.

\section{Appendix A. Classification of cuspidal character sheaves}

Let $\mathbf{G}$ be a connected reductive algebraic group over an algebraically closed field of characteristic $p$ (we allow $p=0$ ).

We summarise here the classification of cuspidal character sheaves on $\mathbf{G}$, which is essentially due to Lusztig (see [L85]). In particular we give a list, conveniently arranged, of cuspidal character sheaves for each isogeny type of quasi-simple group.

As explained in 3.1, a cuspidal character sheaf $A$ is the perverse extension of an irreducible cuspidal local system whose support is the inverse image in $\mathbf{G}$ of a conjugacy class of $\mathbf{G} / Z_{\mathbf{G}}^{\circ}$, where $Z_{\mathbf{G}}$ is the centre of $\mathbf{G}$. If $x$ is in the support of $A$ then by $[\mathrm{L} 84 \mathrm{~b}, 2.8]$ the group $C_{\mathbf{G}}^{0}(x) / Z_{\mathbf{G}}^{\circ}$ is unipotent; in particular if $x_{s} x_{u}$ is the Jordan decomposition of $x$ the semi-simple part $x_{s}$ is isolated. The class of $x_{u}$ is distinguished in $C_{\mathbf{G}}^{0}\left(x_{s}\right)$ and $Z_{\mathbf{G}} / Z_{\mathbf{G}}^{\circ}$ injects into $A_{\mathbf{G}}(x)=C_{\mathbf{G}}(x) / C_{\mathbf{G}}^{0}(x)$. By the cleanness of cuspidal character sheaves (see [L12]) $A$ vanishes outside the support of $\mathcal{E}$.

As explained in 3.3 , a cuspidal character sheaf has a "Lusztig series", parameterised by the conjugacy class of some semi-simple element $s$ of the Langlands dual group $\mathbf{G}^{*}$ to $\mathbf{G}$; the sheaf further defines a label inside a family attached to the group $W^{\prime}(s)=W_{\mathbf{G}^{*}}(s)$. Specifically, the group $W^{\prime}(s)$ is of the form $W(s) \rtimes \Omega$ where $W(s)=W\left(C_{\mathbf{G}^{*}}^{0}(s)\right)$ and where $\Omega \simeq A_{\mathbf{G}^{*}}(s)$; a family of $W^{\prime}(s)$ with a label attached to a cuspidal character sheaf is determined by an $\Omega$-stable family of $W(s)$; if the small finite group giving rise to the labels of this last family is $\mathcal{G}$, the group giving rise to the labels of the family of $W^{\prime}(s)$ is $\mathcal{G} \rtimes \Omega$ (see [L85, 17.1-17.8]). There is at most one family in a given group $W(s)$ arising as above ([L84, Chapter 8]). The labels for the cuspidal character sheaves in the family are pairs $(x, \chi)$ taken up to $\mathcal{G} \rtimes \Omega$-conjugacy where $x \in \mathcal{G} \rtimes \Omega$ and $\chi \in \operatorname{Irr}\left(C_{\mathcal{G} \rtimes \Omega}(x)\right)$. We will call cuspidal labels the labels which can be labels for cuspidal character sheaves. 
To each irreducible local system on the class of $x$ is associated a central character, coming from the action of $Z_{\mathbf{G}}$ on fibres; it is also the restriction to $Z_{\mathbf{G}}$ of the character of $A_{\mathbf{G}}(s u)$ associated to the local system.

We will use the following facts

- The cuspidal character sheaves on a direct product of groups are the external tensor product of cuspidal character sheaves on each component ([L85, 17.11]).

- The cuspidal character sheaves on $\mathbf{G}$ are obtained from those on $\mathbf{G} / Z_{\mathbf{G}}^{\circ}$ by inverse image and tensoring by a local system $\mathcal{S}$ of rank 1 on $\mathbf{G}$ which is the inverse image of a Kummer system on the abelianisation $\mathbf{G} /[\mathbf{G}, \mathbf{G}]$. The effect on the Lusztig series of tensoring by $\mathcal{S}$ is to multiply the semi-simple element of $\mathbf{G}^{*}$ by the central element corresponding to $\mathcal{S}$ (see Lemma 5.1 or [L85, 17.9, 17.10]).

- Let $\tilde{\mathbf{G}} \stackrel{\pi}{\rightarrow} \mathbf{G}$ be a surjective morphism with a finite central kernel; then, given an irreducible cuspidal local system $\mathcal{S}$ on $\tilde{\mathbf{G}}$, the direct image $\pi_{*} \mathcal{E}$ is a local system if $\mathcal{E}$ is $\operatorname{ker} \pi$-invariant (equivalently its central character vanishes on $\operatorname{ker} \pi$ ); in this latter case, the components of $\pi_{*} \mathcal{E}$ are irreducible cuspidal local systems and all cuspidal local systems on $\mathbf{G}$ are obtained this way [L84b, 2.10]. If $x$ is in the support of $\mathcal{E}$, the direct image $\pi_{*}$ corresponds to the induction through the natural morphism $A_{\tilde{\mathbf{G}}}(x) \rightarrow A_{\mathbf{G}}(\pi(x))$. If $\tilde{\mathbf{G}}^{*} \stackrel{\check{\pi}}{\leftarrow} \mathbf{G}^{*}$ is the dual map, and $\mathcal{E}^{\prime}$ is a component of $\pi_{*} \mathcal{E}$ with Lusztig series $s$, then the Lusztig series of $\mathcal{E}$ is $\check{\pi}(s)$ [L85, 17.16].

The above facts in principle reduce the classification to the case of quasisimple and simply connected groups. But in practice the list is much easier to use if the classification is given for each isogeny type of quasi-simple group, and this is what we will do.

We assume now $\mathbf{G}$ quasi-simple. We use the additional facts

- The central character associated to a cuspidal character sheaf determines the part "element of $\Omega$ " of its label [L85, 23.0].

- For $z \in Z_{\mathbf{G}}$, the associated translation operator $t_{z}$ preserves the Lusztig series [L85, 17.17.2] and acts on the part "character of $\Omega$ " of the label as follows: if the label of $A$ is $(x, \chi)$ and the central character of $A$ is faithful, and $\sigma_{z}$ is the character of $\Omega$ determined by $z$, then the label of $t_{z}^{*} A$ is $\left(x, \chi \sigma_{z}\right)$ [L85, 23.1 (c)]. 
To describe cuspidal character sheaves whose support contains $x$, we give in order a description of $x_{s}$, of $x_{u}$ and of $A_{\mathrm{G}}(x)$ (often it is sufficient for this to describe $C_{\mathbf{G}}\left(x_{s}\right), A_{\mathbf{G}}\left(x_{s}\right)$ and the class of $x_{u}$ in $\left.C_{\mathbf{G}}\left(x_{s}\right)^{0}\right)$, and finally describe the associated character of $A_{\mathbf{G}}(x)$. Note that the class of $x_{u}$ is determined by the class of $x_{s}$ and the fact that the class of $x$ supports a cuspidal local system if and only if the class of $x_{u}$ in $C_{G}\left(x_{s}\right)^{0}$ supports a cuspidal local system (see [L84b, 2.10]).

We also need the following

Lemma A.1. Let $\pi: \tilde{\mathbf{G}} \rightarrow \mathbf{G}$ be surjective morphism with central kernel; let $\tilde{x} \in \tilde{\mathbf{G}}$ and let $x=\pi(\tilde{x})$; there is then an exact sequence

$$
1 \rightarrow \operatorname{ker} \pi /\left(\operatorname{ker} \pi \cap C_{\tilde{\mathbf{G}}}(\tilde{x})^{\circ}\right) \rightarrow A_{\tilde{\mathbf{G}}}(\tilde{x}) \stackrel{\bar{\pi}}{\rightarrow} A_{\mathbf{G}}(x) \stackrel{\eta}{\rightarrow} \operatorname{ker} \pi,
$$

where $\bar{\pi}$ is induced by $\pi$ and where, if $g \in C_{\mathbf{G}}(x)$ is both a representative of $\bar{g} \in A_{\mathbf{G}}(x)$ and the image of $\tilde{g} \in \tilde{\mathbf{G}}$, we define $\eta(\bar{g})=z$ where $z$ is the element defined by ${ }^{\tilde{g}} \tilde{x}=\tilde{x} z$. The map $\bar{\pi}$ is surjective when $x$ is unipotent.

If in addition the $\mathbf{G}$ - conjugacy class of $x$ affords a cuspidal local system then $\left(\operatorname{ker} \pi \cap C_{\tilde{\mathbf{G}}}(\tilde{x})^{\circ}\right)=1$,

Proof. We first observe that the formula for $\eta(\bar{g})$ gives a well-defined element $z \in \operatorname{ker} \pi$, whence a morphism $C_{\mathbf{G}}(x) \rightarrow \operatorname{ker} \pi$ compatible with the quotient by $C_{\mathbf{G}}(x)^{\circ}$ and whose kernel is by definition the image of $C_{\tilde{\mathbf{G}}}(\tilde{x})$, whence an exact sequence

$$
A_{\tilde{\mathbf{G}}}(\tilde{x}) \stackrel{\bar{\pi}}{\rightarrow} A_{\mathbf{G}}(x) \stackrel{\eta}{\rightarrow} \operatorname{ker} \pi .
$$

As $\pi\left(C_{\tilde{\mathbf{G}}}(\tilde{x})^{\circ}\right)=C_{\mathbf{G}}(x)^{\circ}$, the kernel of $\bar{\pi}$ is $\operatorname{ker} \pi / \operatorname{ker} \pi \cap C_{\tilde{\mathbf{G}}}(\tilde{x})^{\circ}$. If $x$ is unipotent, taking the semi-simple parts on both sides of the equality $\tilde{g}_{\tilde{x}} \tilde{x} \tilde{x} z$ we find $z=1$.

When the class of $\tilde{x}$ affords a cuspidal local system the group $C_{\tilde{\mathbf{G}}}(\tilde{x})^{\circ}$ is unipotent which implies that $\operatorname{ker} \pi \cap C_{\tilde{\mathbf{G}}}(\tilde{x})^{\circ}=1$, whence the result.

We use Lemma A.1 as follows: given a local system $\mathcal{E}$ on the class of $\tilde{x}$ lifting a local system on the class of $x$, or equivalently a representation of $A_{\tilde{\mathbf{G}}}(\tilde{x})$ factoring through a representation $\rho$ of $\bar{\pi}\left(A_{\tilde{\mathbf{G}}}(\tilde{x})\right)$, then $\pi_{*} \mathcal{E}$ corresponds to the induced of $\rho$ to $A_{\mathbf{G}}(x)$. We will use two special cases: if the image of $\eta$ is trivial, then $\pi_{*}(\mathcal{E})$ is irreducible; if the image of $\eta$ is of prime cardinality $r$ then either $\pi_{*} \mathcal{E}$ is irreducible or has $r$ irreducible components (depending whether $\rho$ is invariant or not by $A_{\mathbf{G}}(x)$ ). 


\section{Appendix B. Classical groups}

In a classical Weyl group, the groups $\mathcal{G}$ attached to families are of the form $(\mathbb{Z} / 2 \mathbb{Z})^{n}$, and the labels in a family are in bijection with Lusztig's "symbols" (pairs of increasing sequences of natural integers taken up to shift, see [L84, Chapter 4]); we will call cuspidal a symbol corresponding to a cuspidal label.

A useful preliminary is the description of cuspidal symbols for $W\left(B_{n}\right)$ and $W\left(D_{n}\right)$. For $W\left(B_{n}\right)$ where $n=d^{2}+d$ the only cuspidal symbol is $(\{0,1, \ldots, 2 d\}, \emptyset)$; It corresponds to the label $(x, \chi)=$ $((-1,1,-1,1, \ldots),(-1,-1,-1,-1, \ldots))$ in $\mathcal{M}(\mathcal{G})$ where $\mathcal{G}=(\mathbb{Z} / 2 \mathbb{Z})^{d}$. For $W\left(D_{n}\right)$ with $n=(d+1)^{2}$ the only cuspidal symbol is $(\{0,1, \ldots, 2 d+1\}, \emptyset)$; $\mathcal{G}$ and $(x, \chi)$ are as above.

\section{Type $A_{n-1}$}

The only group of type $A_{n-1}$ which affords cuspidal character sheaves is $\mathbf{G}=$ $\mathrm{SL}_{n}$ when $p$ does not divide $n$. There are $n \phi(n)$ such sheaves, parameterised by the pairs $(z, \chi)$ where $z \in Z_{\mathbf{G}}$ and $\chi$ is an injective character of $\chi \in$ $\operatorname{Irr}\left(Z_{\mathbf{G}}\right)$. The cuspidal local system associated to $(z, \chi)$ has support $z C$ where $C$ is the regular unipotent class, and $\chi$ can be identified to the character of $A_{\mathbf{G}}(x)=Z_{\mathbf{G}}$ where $x \in z C$ associated to the local system. These character sheaves are all in the Lusztig series defined by a quasi-isolated $s \in \mathbf{G}^{*}$ such that $W^{\prime}(s)$ contains a Coxeter element of $W\left(\mathbf{G}^{*}\right)$; we have $W(s)=1$ and $\Omega=W^{\prime}(s)$ is in bijection with $\operatorname{Irr}\left(Z_{\mathbf{G}}\right)$. Then $z \in Z_{\mathbf{G}}$ corresponds to $\zeta \in$ $\operatorname{Irr}(\Omega)$ and $\chi$ to a generator $x \in \Omega$, and $(x, \zeta)$ is the label in $\mathcal{M}(\Omega)$ of the considered sheaf (see [L85, 18.5]).

\section{Types $B_{n}$ and $C_{n}$ for $p=2$}

The description is the same in both cases; there are cuspidal character sheaves only when $n / 2$ is a triangular number, in which case there is one [L85, 22.2]. Its support is the class of a unipotent $x_{u}$ of Jordan type (in $\mathrm{Sp}_{2 n}$, see[LS, 2.7]) given by $(4,8,12, \ldots)$; this partition has $d$ parts where $n=d(d+1)$. We have $A_{\mathbf{G}}\left(x_{u}\right)=(\mathbb{Z} / 2 \mathbb{Z})^{d}$ and the local system corresponds to the character $(-1,1,-1,1, \ldots)$. The symbol (in the sense of [LS, 2.7 and 1.2e]) of the local system is $(\{0,4, \ldots, 4 d\}, \emptyset)$ when $d$ is odd and $(\emptyset,\{2, \ldots, 2+4(d-1)\})$ when $d$ is even. The Lusztig series is $\overline{\mathbb{Q}}_{\ell}$ (the unipotent series) and the label is the cuspidal symbol of $W\left(B_{n}\right)$ (see. [L85, $22.4,22.6])$. 


\section{Type $C_{n}$ for $p \neq 2$}

It is useful to first describe the cuspidal local systems with unipotent support. For $C_{n}^{\text {ad }}$ there exists such a system when $n$ is an even triangular number. For $C_{n}^{\text {sc }}$ there is also such a system for $n$ an odd triangular number, which has a nontrivial central character. In each case the support is described by the Jordan type $(2,4,6, \ldots, 2 d)$ where $n=d(d+1) / 2$ and $A_{\mathbf{G}}\left(x_{u}\right)=$ $(\mathbb{Z} / 2 \mathbb{Z})^{d-1}$; the local system corresponds to the character $(-1,1,-1,1, \ldots)$ of $A_{\mathbf{G}}\left(x_{u}\right)$ and the symbol of the local system is $(\{0,2,4, \ldots, 2 d\}, \emptyset)$ if $d$ is even and $(\emptyset,\{1,3,5, \ldots, 2 d+1\})$ otherwise (see [L84b, 11.5 and 12.4]).

Case $C_{n}^{\text {ad }}=\mathbf{P S p}_{2 n}$. $\operatorname{PSp}_{2 n}$ affords cuspidal local systems if and only if $n$ is even and of the form $n=N_{1}+N_{2}$ where $N_{1}$ and $N_{2}$ are triangular numbers (of the same parity). If $N_{1} \neq N_{2}$ there exists a unique cuspidal local system with support the class of $x$ where $C_{\mathbf{G}}\left(x_{s}\right) \simeq \mathrm{Sp}_{2 N_{1}} \times \mathbb{Z} / 2 \mathbb{Z} \mathrm{Sp}_{2 N_{2}}$. If $N_{1}=N_{2}$ there are two cuspidal local systems on the class of $x$ where $C_{\mathbf{G}}\left(x_{s}\right) \simeq\left(\operatorname{Sp}_{2 N_{1}} \times \mathbb{Z} / 2 \mathbb{Z} \mathrm{Sp}_{2 N_{1}}\right) \rtimes \mathbb{Z} / 2 \mathbb{Z}$; the non-trivial element of $A_{\mathbf{G}}\left(x_{s}\right)$ exchanges the two Sp components, and the two local systems correspond to the two characters of $A_{\mathbf{G}}\left(x_{s}\right)$ (see [L85, 23.2(a)]; by [L84b, 2.10] the local system corresponds to a unipotently supported cuspidal local system on $C_{\mathbf{G}}\left(x_{s}\right)^{0}$, of central character Id $\otimes \mathrm{Id}$ when $N_{1}$ and $N_{2}$ are even, and of central character $\varepsilon \otimes \varepsilon$ when $N_{1}$ and $N_{2}$ are odd). If $N_{1}=t(t+1) / 2$ and $N_{2}=r(r+1) / 2$ with $t, r \geq 0$, then the Lusztig series is given by $s \in$ $\operatorname{Spin}_{2 n+1}$ such that $C_{\mathbf{G}^{*}}(s) \simeq \operatorname{Spin}_{2 A} \times^{\mathbb{Z} / 2 \mathbb{Z}} \operatorname{Spin}_{4 B+1}$ where if $t \not \equiv r(\bmod 2)$ we have $4 A=(t+r+1)^{2}$ and $8 B+1=(t-r)^{2}$ and if $t \equiv r(\bmod 2)$ we have $4 A=(t-r)^{2}$ and $8 B=(t+r+2)(t+r)$ (see [L92, p. 976] and [L85, 23.16]). The above describes a unique element $s$ if $t \neq r$ and two (central) elements if $t=r$. The label is given by the cuspidal symbol of the group $W(s) \simeq W\left(D_{A}\right) \times W\left(B_{2 B}\right)$ (with the convention that $W\left(D_{1}\right)$ is the trivial group).

Case $C_{\boldsymbol{n}}^{\mathbf{s c}}=\mathbf{S p}_{\mathbf{2 n}}$. Again we must have $n=N_{1}+N_{2}$ where $N_{1}$ and $N_{2}$ are triangular numbers. If $n$ is even and $N_{1} \neq N_{2}$ there are two local cuspidal systems, inverse images of the one in $C_{n}^{\text {ad }}$, where the semi-simple part of the support is respectively $x_{s}$ and $x_{s} z$, where $z$ is the non-trivial element of $Z \mathbf{G}$. If $N_{1}=N_{2}$ the element $x_{s}$ is conjugate to $x_{s} z$, and $C_{\mathbf{G}}\left(x_{s}\right)$ is connected, which leads to a single local system. If $N_{1} \not \equiv N_{2}(\bmod 2)$ there are also two cuspidal local systems, with a nontrivial central character (see [L85, 23.2 (b)]). In every case one can index the cuspidal local systems by ordered pairs $\left(N_{1}, N_{2}\right)$; we have $C_{\mathbf{G}}\left(x_{s}\right) \simeq \mathrm{Sp}_{2 N_{1}} \times \mathrm{Sp}_{2 N_{2}}$. If $t, r, A, B$ are defined by 
the same formula as in the previous subsection, the Lusztig series is given by an element $s$ such that $C_{\mathbf{G}^{*}}(s) \simeq \mathrm{O}_{2 A} \times \mathrm{SO}_{4 B+1}$. The group $\Omega$ is thus $\mathbb{Z} / 2 \mathbb{Z}$ excepted if $N_{1}=N_{2}$ (the element of $\Omega$ part of the label corresponds to the semisimple part $x_{s}$ or $x_{s} z$ of the support of the local system). The part "in $W(s)$ " of the label is the cuspidal symbol of the group $W(s) \simeq$ $W\left(D_{A}\right) \times W\left(B_{2 B}\right)$.

\section{Type $B_{n}$ for $p \neq 2$}

It is useful to first describe the cuspidal local systems with unipotent support. For $B_{n}^{\text {ad }}$ there is a (unique) such system when $2 n+1$ is a square (see [L84b, 13.4]). For $B_{n}^{\text {sc }}$, there is an additional system for $2 n+1$ a triangular number, with a nontrivial central character [L84b, 14.6]. In the first case the Jordan type of the support is $(1,3,5, \ldots)$ and if $d$ is the number of parts of this partition then $A_{\mathrm{SO}}\left(x_{u}\right)=(\mathbb{Z} / 2 \mathbb{Z})^{d-1}[\mathrm{~L} 84 \mathrm{~b}, 10.6]$; the local system corresponds to the character $(-1,1,-1, \ldots)$ of this group. We have $n=d^{2}$ and the symbol (in the sense of [L84b, 13.4]) of the local system is $(\{0,2,4, \ldots, 2 d-2\}, \emptyset)$. In the second case the Jordan type of the support is $(1,5,9, \ldots)$ or $(3,7,11, \ldots)$. We have $A_{\mathbf{G}}\left(x_{u}\right)=(\mathbb{Z} / 2 \mathbb{Z})^{d}$ where $d$ is the number of parts of the Jordan type (see [L84b, 10.6] and Lemma A.1].

Case $\boldsymbol{B}_{\boldsymbol{n}}^{\mathbf{a d}}=\mathbf{S O}_{\mathbf{2 n + 1}}$. We must have $2 n+1=N_{1}+N_{2}$ where $N_{1}$ is an even square and $N_{2}$ an odd square. There is one cuspidal local system, unipotently supported, when $N_{1}=0$, and two cuspidal local systems if $N_{1} \neq$ 0 (see [L85, 23.2(c)]); in this last case we have $C_{\mathbf{G}}\left(x_{s}\right) \simeq \mathrm{O}_{N_{1}} \times \mathrm{SO}_{N_{2}}$. Let $N_{1}=r^{2}$ and $N_{2}=t^{2}$; then the Lusztig series is defined by $s$ of centraliser $\mathrm{Sp}_{\left((r+t)^{2}-1\right) / 2} \times \mathrm{Sp}_{\left((r-t)^{2}-1\right) / 2}$.

Case $\boldsymbol{B}_{n}^{\text {sc }}=\operatorname{Spin}_{2 n+1}$. Concerning systems coming from $B_{n}^{\text {ad }}$ (with a trivial central character) if $N_{1}$ and $N_{2}$ as above are distinct and both nonzero, the preimages $x_{s}$ and $x_{s} z$ are conjugate and we have a single local system. If $N_{1}=0$ there are two systems, one with unipotent support, the other translated by the non-trivial element of the centre. When the central character is nontrivial (see [L85, 23.2 (e)]), we must have $2 n+1=N_{1}+N_{2}$ where $N_{1}$ is an even triangular numbers and $N_{2}$ an odd triangular number. Each such pair gives rise to two cuspidal complexes. When $N_{1} \neq 0$ and $N_{2} \neq 1$, they share the same support, the class of $x$ where $C_{\mathbf{G}}\left(x_{s}\right) \simeq \operatorname{Spin}_{N_{1}} \times \mathbb{Z} / 2 \mathbb{Z} \operatorname{Spin}_{N_{2}}\left(x_{s}\right.$ and $x_{s} z$ are conjugate); $\operatorname{Spin}_{N_{1}}$ has two central characters which restrict to a nontrivial character of $Z_{\mathbf{G}}$, corresponding to the two local systems. If $N_{2}=1$ we have 
$C_{\mathbf{G}}\left(x_{s}\right) \simeq \operatorname{Spin}_{N_{1}}$ and if $N_{1}=0$ one of them is unipotently supported and the other translated by the non-trivial element of the centre. Let $\{r, t\}$ be positive integers such that $\left\{N_{1}, N_{2}\right\}=\{r(r+1) / 2, t(t+1) / 2\}$. If $r$ have $t$ different parity, let $t$ be the even one. Then we must have $r \equiv t+1$ $(\bmod 4)\left(\right.$ for $N_{1}+N_{2}$ to be odd), and we let $A=(r+t+3)(r+t-1) / 16$ and $B=(r-t-1)(r-t+1) / 8$. If $r$ and $t$ have same parity we must have $r \equiv t+2(\bmod 4)$ and we let $A=(r-t-2)(r-t+2) / 16$ and $B=$ $(r+t)(r+t+2) / 8$. The Lusztig series (see [L92, 1.11]) is then defined by $s \in \mathbf{G}^{*}$ such that $C_{\mathbf{G}^{*}}(s) \simeq\left(\left(\mathrm{Sp}_{2 A} \times \mathrm{GL}_{B} \times \mathrm{Sp}_{2 A}\right) /(\mathbb{Z} / 2 \mathbb{Z})\right) \rtimes \mathbb{Z} / 2 \mathbb{Z}$ where the nontrivial element of $A_{\mathrm{G}^{*}}(s)$ exchanges the two Sp components and induces the transpose inverse automorphism of the GL component.

\section{Type $D_{n}$ for $p=2$}

There is at most one cuspidal character sheaf, and it occurs when $n$ is an even square [L85, 22.3]. The support is unipotent of Jordan type $(2,6,10, \ldots, 4 d-$ 2 ); this partition has $d$ parts where $n=d^{2}$ (see [LS, 3.3 and 1.2e]); we have $A_{\mathbf{G}}\left(x_{u}\right) \simeq(\mathbb{Z} / 2 \mathbb{Z})^{d-1}$ and the symbol in the sense of loc. cit. of the local system is $(\{0,4, \ldots, 4 d\}, \emptyset)$. The Lusztig series is $\overline{\mathbb{Q}}_{\ell}$ (unipotent series) and the label is the cuspidal symbol of $W\left(D_{n}\right)$ (see [L85, 22.7]).

\section{Type $D_{n}$ for $p \neq 2$}

It is useful to first describe the cuspidal local systems with unipotent support. For $D_{n}^{\text {ad }}$ there is such a system exactly when $2 n$ is a square and $n / 2$ is even. For $\mathrm{SO}_{2 n}$, there an additional system when $2 n$ is a square and $n / 2$ is odd, with a nontrivial central character. For $\operatorname{Spin}_{2 n}=D_{n}^{\text {sc }}$ there is additionally a system when $2 n$ is a triangular number, attached to each of the two central characters which are nontrivial on an element of the kernel of $\operatorname{Spin}_{2 n} \rightarrow \mathrm{SO}_{2 n}$. If $2 n=d^{2}$ the support $x_{u}$ has Jordan type $(1,3,5, \ldots, 2 d-$ $1)$, we have $A_{\mathrm{SO}}\left(x_{u}\right)=(\mathbb{Z} / 2 \mathbb{Z})^{d-1}([\mathrm{~L} 84 \mathrm{~b}, 10.6])$ and the symbol in the sense of [L84b, 13.4] of the local system is $(0,2,4, \ldots, 2 d-2, \emptyset)$. If $4 n=d(d+1)$ with $d$ odd (resp. even), the Jordan type of the support is $(1,5,9, \ldots, 2 d-1)$ (resp. $(3,7,11, \ldots, 2 d-1)$ ), (see [LS, 4.9]). In these latter cases $A_{\mathbf{G}}\left(x_{u}\right)$ is a nonsplit central extension by $\mathbb{Z} / 2$ of $A_{\mathrm{SO}}\left(x_{u}\right)=(\mathbb{Z} / 2 \mathbb{Z})^{\left[\frac{d-1}{2}\right]}$ and the two cuspidal local systems correspond to the two characters of degree $2^{\left[\frac{d-1}{4}\right]}$ of this group $([\mathrm{L} 84 \mathrm{~b}, 14.3,14.4])$ (note that $d \equiv 0$ or $3(\bmod 4)$, thus $\left[\frac{d-1}{2}\right]$ is odd). Note also that when $n$ is even the unipotently supported cuspidal local system are the preimage of those of $\frac{1}{2} \operatorname{Spin}_{2 n}$. 
Case $D_{n}^{\text {ad }}=\mathbf{P S O}_{2 n} . \quad \mathrm{PSO}_{2 n}$ affords cuspidal local systems if and only if $n$ is a multiple of 4 and $2 n=N_{1}+N_{2}$ where $\left(N_{1}, N_{2}\right)$ is an unordered pair of even squares. If $N_{1}$ or $N_{2}$ is zero it affords one cuspidal system, otherwise if $N_{1}$ and $N_{2}$ are nonzero and distinct there are two cuspidal systems. If $N_{1}=$ $N_{2} \neq 0$ there are 4 cuspidal systems (see [L85, 23.2 (c)]). These systems all have the same support, the class of $x$ where $C_{\mathbf{G}}\left(x_{s}\right)^{\circ} \simeq \mathrm{SO}_{N_{1}} \times \mathbb{Z} / 2 \mathbb{Z} \mathrm{SO}_{N_{2}}$. If $N_{1}$ or $N_{2}$ is zero then $x_{s}=1$, otherwise if $N_{1} \neq N_{2}$ we have $A_{\mathbf{G}}\left(x_{s}\right)=\mathbb{Z} / 2 \mathbb{Z}$, acting by the outer automorphism on both factors, and if $N_{1}=N_{2} \neq 0$ we have $A_{\mathbf{G}}\left(x_{s}\right) \simeq \mathbb{Z} / 2 \mathbb{Z} \times \mathbb{Z} / 2 \mathbb{Z}$ where the second factor $\mathbb{Z} / 2 \mathbb{Z}$ exchanges the two $S O_{N_{1}}$ factors of $C_{\mathbf{G}}\left(x_{s}\right)^{\circ}$.

The Lusztig series is defined by $s$ such that $C_{\mathbf{G}^{*}}(s)$ affords the double cover $\operatorname{Spin}_{2 a^{2}} \times \operatorname{Spin}_{2 b^{2}}$ where $a \geq 0$ and $b \geq 0$ are given by $N_{1}=(a+b)^{2}$ and $N_{2}=(a-b)^{2}$ (see [L92, 1.12] or [L85, $\left.\left.23.19\left(\mathrm{c}_{2}\right)\right]\right)$. There are as many such semi-simple classes as cuspidal local systems, and each system lies in a different series. The label of a cuspidal system is the cuspidal symbol of the group $W\left(D_{a^{2}}\right) \times W\left(D_{b^{2}}\right)$.

Case $\mathbf{S O}_{2 n} \cdot \quad \mathrm{SO}_{2 n}$ affords cuspidal local systems if and only if $2 n=N_{1}+$ $N_{2}$ where $N_{1}$ and $N_{2}$ are even squares. If $n \equiv 0(\bmod 4)$ the cuspidal systems are a preimage from $D_{n}^{\text {ad }}$ and have a trivial central character (using Lemma A.1). If $n \equiv 2(\bmod 4)$ they have a nontrivial central character (see [L85, 23.2 (d) and 23.19 (d)]).

A description which covers both cases is as follows: for each ordered pair $\left(N_{1}, N_{2}\right)$ there is a cuspidal system if $N_{1}=0$ or $N_{2}=0$ and two cuspidal systems otherwise. The supports are the class of $x$ such that $C_{\mathbf{G}}\left(x_{s}\right)^{\circ} \simeq$ $\mathrm{SO}_{N_{1}} \times \mathrm{SO}_{N_{2}}$ : there are two such classes if $N_{1} \neq N_{2}$ (which is always the case if $n \equiv 2(\bmod 4))$, that we parameterise repectively by $\left(N_{1}, N_{2}\right)$ and $\left(N_{2}, N_{1}\right)$, and only one class if $N_{1}=N_{2}$. If $N_{1}$ and $N_{2}$ are nonzero $A_{\mathrm{G}}\left(x_{s}\right)$ is $\mathbb{Z} / 2$ acting by the simultaneous exterior automorphism of both components. If $N_{1} \neq N_{2}$ the translation by the centre exchanges $N_{1}$ and $N_{2}$ (exchanging the supports). If $N_{1}=N_{2}$, the centre acts trivially (then $\left.n \equiv 0(\bmod 4)\right)$. We have $x_{s}=1$ if $N_{1}=0$ and $x_{s}$ a nontrivial central element if $N_{2}=0$. The Lusztig series is described by $s$ such that $C_{\mathbf{G}^{*}}^{\circ}(s) \simeq \mathrm{SO}_{2 a^{2}} \times \mathrm{SO}_{2 b^{2}}$ where $a$ and $b$ are defined by the same formulae as in the $\mathrm{PSO}_{2 n}$ case excepted that $b$ dmay have an arbitrary sign. Note that if $N \equiv 2(\bmod 4), a$ and $b$ must be odd. There is two such classes excepted if $a= \pm b$, that is $N_{1}=0$ or $N_{2}=0$. If $a$ and $b$ are nonzero we have $A_{\mathrm{G}^{*}}(s) \simeq \mathbb{Z} / 2 \mathbb{Z}$ acting by the simultaneous exterior automorphism of $\mathrm{SO}_{2 a^{2}}$ and $\mathrm{SO}_{2 b^{2}}$.

Case $\frac{1}{2} \operatorname{Spin}_{2 n}$. There exist cuspidal systems with a non-trivial central character (see [L85, 23.2 (f)]) if and only if $n \geq 6$ is odd, of the form $2 n=$ 
$N_{1}+N_{2}$ where $N_{1}$ and $N_{2}$ are even triangular numbers. For each such non ordered pair $\left(N_{1}, N_{2}\right)$ there are two cuspidal local systems if $N_{1} \neq N_{2}$ and four otherwise (see [L85, 23.2 (f)]). If $N_{1}$ and $N_{2}$ are non zero the systems have all support the class of $x$ where $C_{\mathbf{G}}\left(x_{s}\right)^{\circ}$ is isogenous to $\mathrm{SO}_{N_{1}} \times \mathrm{SO}_{N_{2}}$. If $N_{1}=0$ one of the two systems has unipotent support and the other is translated by the nontrivial element of the centre. In any case the action of the centre is free on the 2 or 4 systems. Let $N_{1}=\frac{r(r+1)}{2}$ and $N_{2}=\frac{t(t+1)}{2}$ with $r, t \geq 0$. Then $r$ and $t$ are equal to 0 or $3(\bmod 4)$.

If $r \not \equiv t(\bmod 4)$ the Lusztig series is defined by $s$ such that $C_{\mathbf{G}^{*}}(s)^{\circ}$ is isogenous to

$$
\mathrm{SO}_{\frac{(r+t+1)^{2}}{8}} \times \mathrm{GL}_{\frac{(r-t)^{2}-1}{8}} \times \mathrm{SO}_{\frac{(r+t+1)^{2}}{8}}
$$

if $r \equiv t(\bmod 4)$ the Lusztig series is defined by $s$ such that $C_{\mathbf{G}^{*}}(s)^{\circ}$ is isogenous to

$$
\mathrm{SO}_{\frac{(r-t)^{2}}{8}} \times \mathrm{GL}_{\frac{(r+t)(r+t+2)}{8}} \times \mathrm{SO}_{\frac{(r-t)^{2}}{8}}
$$

(see $\left[\right.$ L85, 23.19 (f)]). There is a unique such class, with $A_{\mathbf{G}^{*}}(s) \simeq \mathbb{Z} / 2 \mathbb{Z}$ acting by interchanging the two SO components.

The other cuspidal local systems have a trivial central character and come from $\mathrm{PSO}_{2 n}$ (we use here Lemma A.1). Then $2 n=N_{1}+N_{2}$ with $N_{1}$ and $N_{2}$ even squares. There is one cuspidal local system for each unordered pair such that $N_{1} \neq N_{2}$ and two systems when $N_{1}=N_{2}$. The support is the class of $x$ where $C_{\mathrm{G}}\left(x_{s}\right)^{\circ}$ is isogenous to $\mathrm{SO}_{N_{1}} \times \mathrm{SO}_{N_{2}}$. If $N_{1}$ or $N_{2}$ is zero then $x_{s}=1$, otherwise if $N_{1} \neq N_{2}$ then $A_{\mathbf{G}}\left(x_{s}\right)=\{1\}$ and if $N_{1}=N_{2} \neq 0$ then $A_{\mathbf{G}}\left(x_{s}\right) \simeq \mathbb{Z} / 2 \mathbb{Z}$ acting by the exchange of the two SO factors. The Lusztig series is defined by $s$ such that $C_{\mathbf{G}^{*}}(s)$ is isogenous to $\mathrm{SO}_{2 a^{2}} \times \mathrm{SO}_{2 b^{2}}$ where $a$ and $b$ are defined as in the PSO case; there is a unique such class. If $a$ and $b$ are distinct (equivalently $N_{1}$ and $N_{2}$ non zero), the centraliser $C_{\mathbf{G}^{*}}(s)$ is connected. If $N_{1}=0$ (that is $a=b$ ) the group $A_{\mathbf{G}^{*}}(s)$ is $\mathbb{Z} / 2$, acting by exchanging the two components.

Case $\operatorname{Spin}_{2 n}$. There are cuspidal local systems coming from $\mathrm{SO}_{2 n}$ or $\mathrm{PSO}_{2 n}$ (see Lemma A.1) only if $n$ is even. As above, let $2 n=N_{1}+N_{2}$ where $N_{1}$ and $N_{2}$ are even squares. For each ordered pair $\left(N_{1}, N_{2}\right)$ with nonzero $N_{1}$ and $N_{2}$, there is a unique cuspidal local system. It comes from $\mathrm{PSO}_{2 n}$ if $n \equiv 0(\bmod 4)$ and from $\mathrm{SO}_{2 n}$ if $n \equiv 2(\bmod 4)$. If $N_{1}=0$ or if $N_{2}=0$ there are two systems, each with the semisimple part of the support central. The lusztig series is defined by $s$ such that $C_{\mathbf{G}^{*}}^{\circ}(s) \simeq \mathrm{SO}_{2 a^{2}} \times{ }^{\mathbb{Z} / 2 \mathbb{Z}} \mathrm{SO}_{2 b^{2}}$, where $N_{1}=(a+b)^{2}$ and $N_{2}=(a-b)^{2}$ with $a \in \mathbb{N} 0$ and $b \in \mathbb{Z}$. The group $A_{\mathbf{G}^{*}}(s)$ is trivial if $N_{1}=N_{2}$, is of order 2 if $N_{1}$ and $N_{2}$ are distinct nonzero and is of order 4 if $N_{1}$ or $N_{2}$ is zero. 
We now look at systems coming from $\frac{1}{2} \operatorname{Spin}_{2 n}$ and not from $\mathrm{PSO}_{2 n}$ (see Lemma A.1): they exist if $n \geq 6$ is even, of the form $2 n=N_{1}+N_{2}$ where $N_{1}$ and $N_{2}$ are even triangular numbers. For each ordered pair $\left(N_{1}, N_{2}\right)$, if $N_{1}$ and $N_{2}$ are non zero there are two cuspidal local system with support the class of $x$ such that $C_{\mathbf{G}}\left(x_{s}\right)$ affords $\operatorname{Spin}_{N_{1}} \times \operatorname{Spin}_{N_{2}}$ as a double cover; there are two such classes if $N_{1} \neq N_{2}$ and only one if $N_{1}=N_{2}$. The cases $N_{1}=0$ and $N_{2}=0$ correspond to 4 cuspidal local systems with semisimple part of the support each one of the 4 central elements. The Lusztig series is defined by $s$ such that, if $N_{1}=\frac{r(r+1)}{2}$ and $N_{2}=\frac{t(t+1)}{2}$, then $C_{\mathrm{G}^{*}}(s)^{\circ} \simeq\left(\mathrm{SO}_{\frac{(r+t+1)^{2}}{8}} \times \mathrm{GL}_{\frac{(r-t)^{2}-1}{8}} \times \mathrm{SO}_{\frac{(r+t+1)^{2}}{8}}\right) /\{ \pm 1\}$ or $\left(\mathrm{SO}_{\frac{(r-t)^{2}}{8}} \times \mathrm{GL}_{\frac{(r+t)(r+t+2)}{8}} \times \mathrm{SO}_{\frac{(r-t)^{2}}{8}}\right)^{8}\{ \pm 1\}$ depending on the values of $r$ and $t(\bmod 4)$ (as in the case of $\left.\frac{1}{2} \mathrm{Spin}\right)$. If $N_{1} \neq N_{2}$ there is a single such class; the group $A_{\mathrm{G}^{*}}(s)$ is noncyclic of order 4 generated by two elements $x$ and $x^{\prime}$ where $x$ acts by the simultaneous outer automorphism of both SO components and $x^{\prime}$ acts by exchanging the two SO components and doing the outer automorphism on the GL component. If $N_{1}=N_{2}$, there are two such classes; the group $A_{\mathrm{G}^{*}}(s)$ is of order 2 acting by the outer automorphism of GL.

Finally we look at the cuspidal local systems whose central character is injective on $Z \mathbf{G}$ (see [L85, 23.2 (e)]): they exist only if $n$ is odd, in which case $Z \mathbf{G}$ has two injective characters. For each of these and for each ordered pair $\left(N_{1}, N_{2}\right)$ of even triangular numbers such that $2 n=N_{1}+N_{2}$ there are two cuspidal local systems (note that $N_{1} \neq N_{2}$ and that $N_{1}$ and $N_{2}$ are nonconsecutive triangular numbers since $n$ is odd). The 4 complexes attached to $\left(N_{1}, N_{2}\right)$ and $\left(N_{2}, N_{1}\right)$ are obtained from each other by translating by the various elements of the centre. If $N_{1}>0$ and $N_{2}>0$ the two systems attached to $\left(N_{1}, N_{2}\right)$ have support the class of $x$ such that $C_{\mathbf{G}}\left(x_{s}\right)$ affords $\operatorname{Spin}_{N_{1}} \times \operatorname{Spin}_{N_{2}}$ as a double cover. If $N_{1}=0$ one of the systems has unipotent support, the other as well as the two systems parameterised by $\left(N_{2}, 0\right)$ are deduced by translation by the centre.

The Lusztig series of a cuspidal local system parameterised by $\left(N_{1}, N_{2}\right)$ is defined by $s$ such that, if $N_{1}=\frac{r(r+1)}{2}$ and $N_{2}=\frac{t(t+1)}{2}$, then $\quad C_{\mathbf{G}^{*}}(s)^{\circ} \simeq\left(\mathrm{SO}_{\frac{(r+t+1)^{2}}{8}} \times \mathrm{GL}_{\frac{(r-t)^{2}-1}{8}} \times \mathrm{SO}_{\frac{(r+t+1)^{2}}{8}}\right) /\{ \pm 1\} \quad$ or $\left(\mathrm{SO}_{\frac{(r-t)^{2}}{8}} \times \mathrm{GL}_{\frac{(r+t)(r+t+2)}{8}} \times \mathrm{SO}_{\frac{(r-t)^{2}}{8}}^{8}\right) /\{ \pm 1\}^{8}$ depending ${ }^{8}$ on the values of $r$ and ${ }^{8}(\bmod 4)$ as in the previous case (see [L92, 1.12] or [L85, $23.19\left(\mathrm{e}_{2}\right)$ ]; note that $r \neq t$ since $N_{1} \neq N_{2}$ and that $|r-t| \neq 1$ since $N_{1}$ and $N_{2}$ are not consecutive). We have $A_{\mathrm{G}^{*}}(s) \simeq \mathbb{Z} / 4 \mathbb{Z}$ where the generator acts by exchanging the two SO components and twisting the GL components, its square twisting the two SO components. 


\section{Appendix C. Exceptional groups}

In the following tables the labels for the cuspidal local systems are, as in [L85, elements of $\mathcal{M}(\mathcal{G})$ for some groups $\mathcal{G}$. We fix notations for the labels $(x, \chi)$ in these groups as follows: in $\mathfrak{S}_{n}$ with $n=2,3,4,5$, we let $g_{i}$ for $i=2,3,4,5$ denote an $i$-cycle; $g_{2}^{\prime}$ denotes the product of two commuting transpositions and $g_{6}$ is an element of order 6 of $\mathfrak{S}_{5}$. We denote by $\theta, \theta^{2}$ (resp. $i,-i$, resp. $-\theta,-\theta^{2}$ ) the injective characters of $\mathbb{Z} / 3 \mathbb{Z}$ (resp. $\mathbb{Z} / 4 \mathbb{Z}$, resp. $\mathbb{Z} / 6 \mathbb{Z}$ ). Finally, when $C_{\mathcal{G}}(x)$ is a Coxeter group (which for instance happens when $\mathcal{G}=\mathfrak{S}_{4}$ and $x \in\left\{1, g_{2}, g_{2}^{\prime}\right\}$ ) we denote by $\varepsilon$ the sign character of this group.

The semi-simple part of the support of the cuspidal local system will be specified by giving the isomorphism type of its centraliser (if needed stating also the number of such conjugacy classes) or by an explicit description. In the case when this centraliser is a product of quasisimple groups with cyclic center, amalgamated by part of their center, we denote $z_{i}$ a generator of the center of the $i$-th factor in order to describe the amalgamation.

It is proved in [L12] that all cuspidal local systems are "clean" in the sense of Lusztig. In bad characteristic we could not find the classification in the litterature but the reader can check that our tables are complete by using the knowledge of unipotently supported cuspidal local systems, of isolated semi-simple elements and the argument [L84b, 2.10.1] of Lusztig.

The parameterisation by labels in families of character sheaves has been worked out in [L85, [S1] ar [S2] except in a few cases marked by "??" in the tables. We have completed this parameterisation so that in every case we have:

Property (*). (see [S1, 6.2], [S2, 4.6] and [Os) The eigenvalue of Shintani ("twisting" operator of Shoji) on the local system parameterised by $(x, \chi)$ is $\chi(x) / \chi(1)$.

In the cases marked "??", it is unknown whether the multiplicity property given in [L85, 17.8.3] holds. 
On Character Sheaves and Characters of Reductive Groups...

503

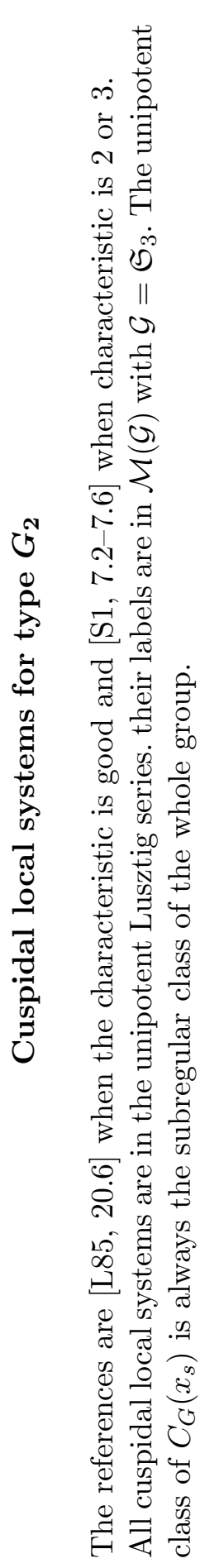

\begin{tabular}{|c|c|c|c|}
\hline 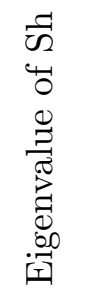 & Ґ & $\tilde{8}$ & $\dashv$ \\
\hline 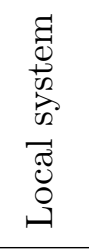 & $\omega$ & $\approx$ & $\omega$ \\
\hline 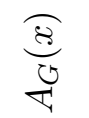 & $\frac{\mathbb{N}}{\mathbb{N}}$ & $\frac{\mathbb{N}}{\mathbb{N}}$ & $\mho^{\infty} \frac{\mathbb{N}}{\mathbb{N}}$ \\
\hline 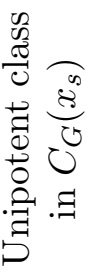 & 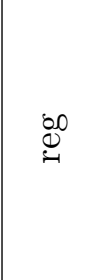 & 惲 & 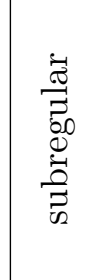 \\
\hline 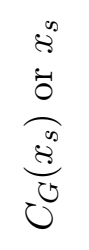 & 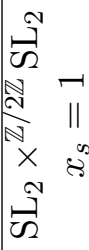 & 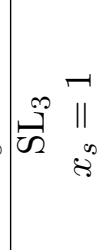 & $\begin{array}{l}71 \\
\| \\
\overbrace{0}^{\infty}\end{array}$ \\
\hline 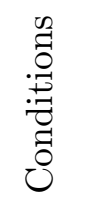 & $\begin{array}{ll}N & N \\
W & 11 \\
2 & 2\end{array}$ & $\begin{array}{cc}\infty & \infty \\
1 & 11 \\
2 & 2\end{array}$ & $\begin{array}{cc}\infty & m \\
k & 11 \\
2 & 2\end{array}$ \\
\hline 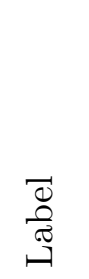 & $\begin{array}{l}\overparen{\omega} \\
\hat{S}\end{array}$ & $\begin{array}{l}\stackrel{N}{i} \\
\| \\
\stackrel{\sigma}{\sigma} \\
\tilde{g}\end{array}$ & 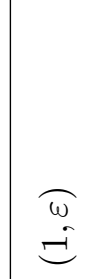 \\
\hline
\end{tabular}



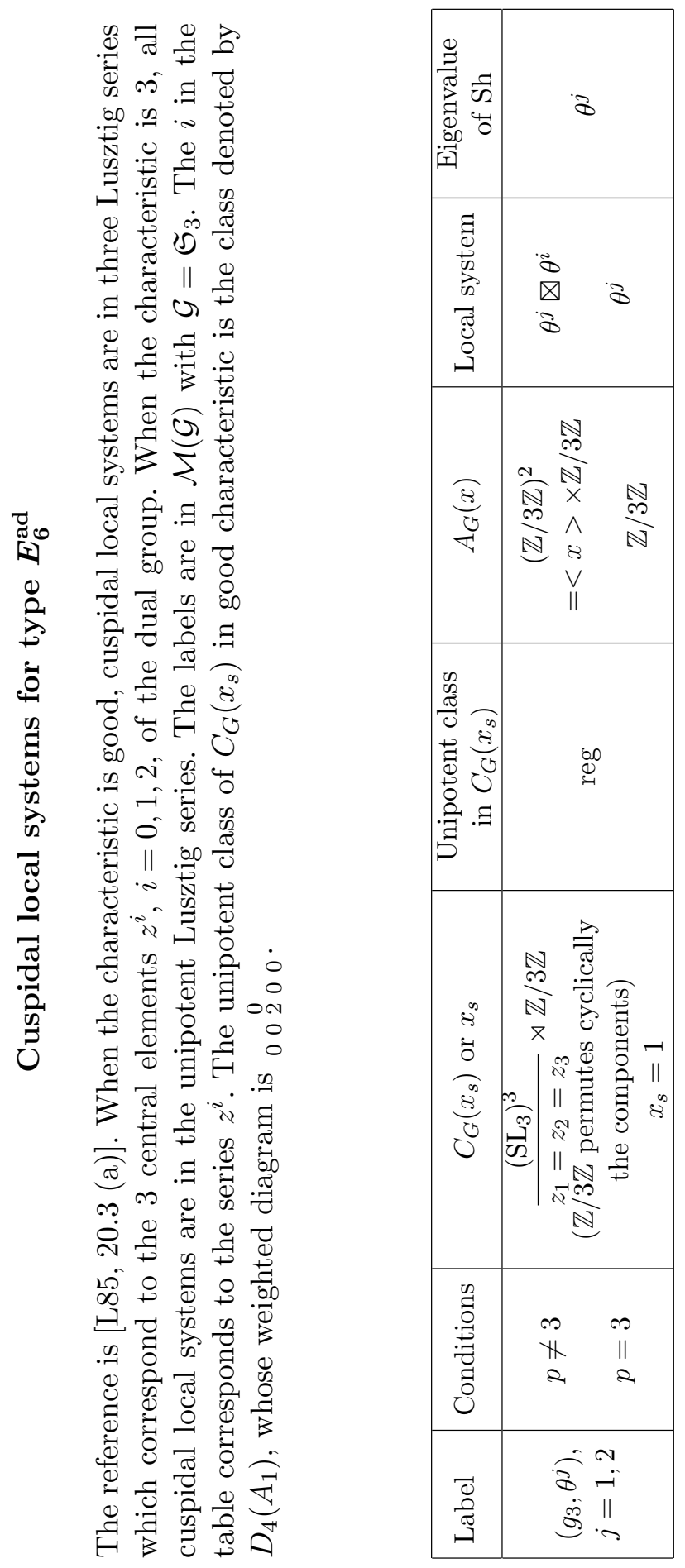
On Character Sheaves and Characters of Reductive Groups...

505

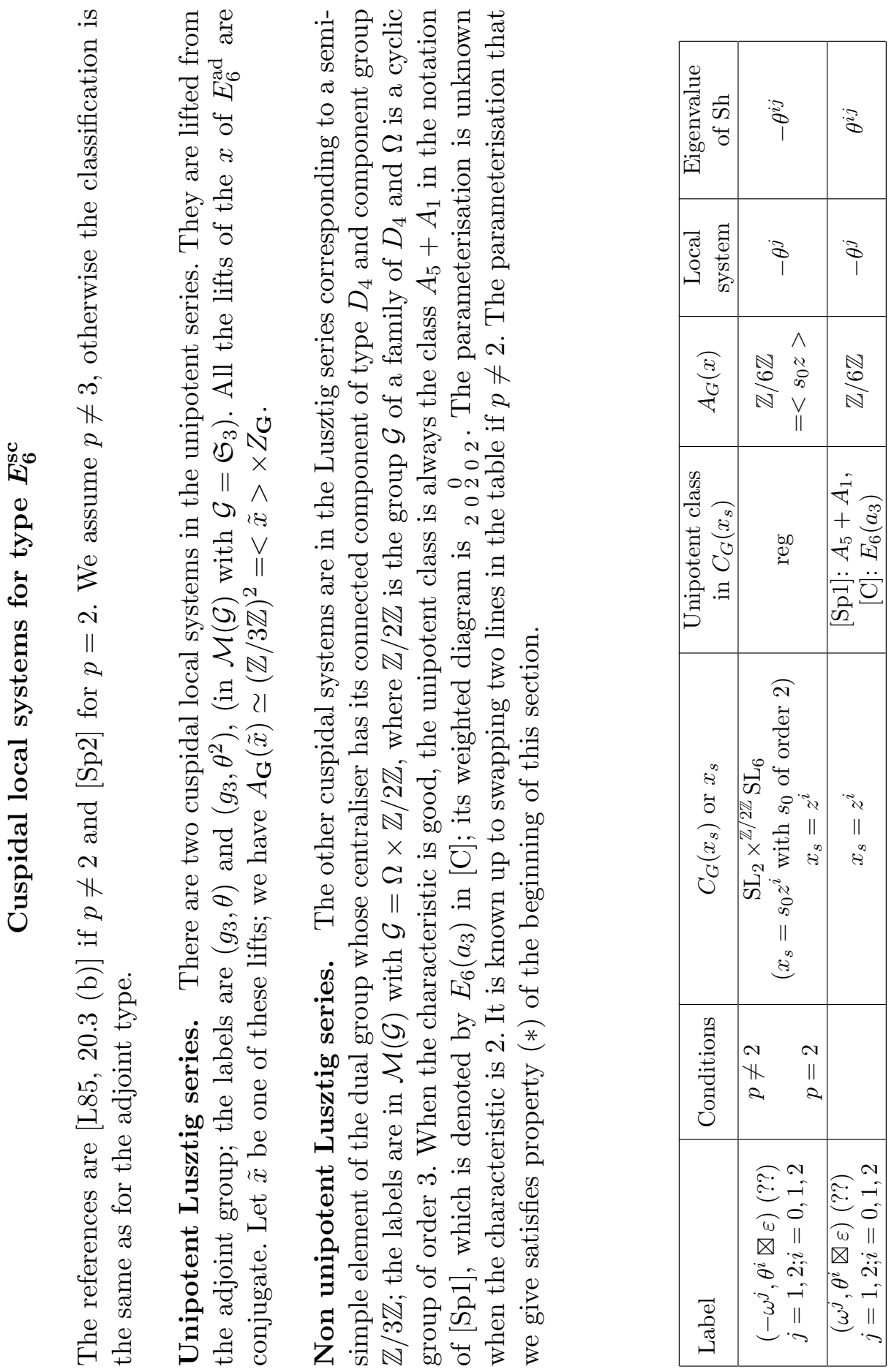




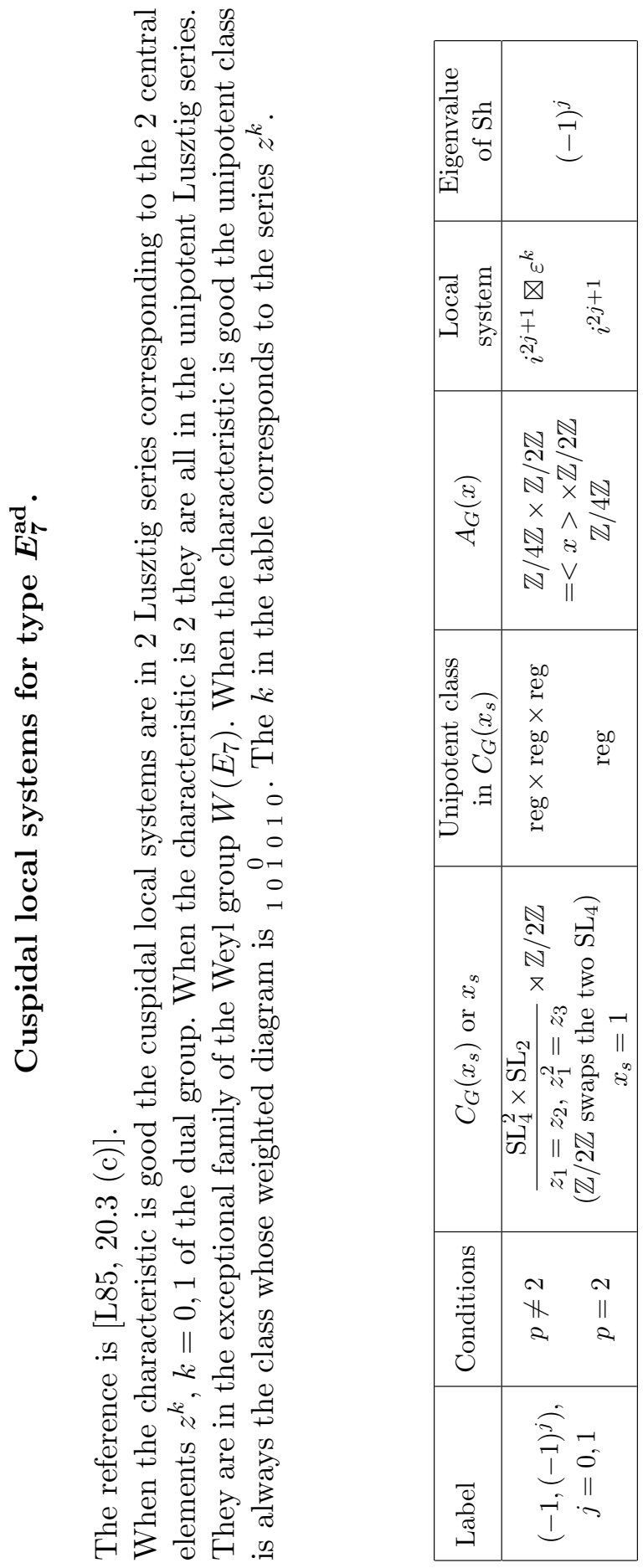


On Character Sheaves and Characters of Reductive Groups...

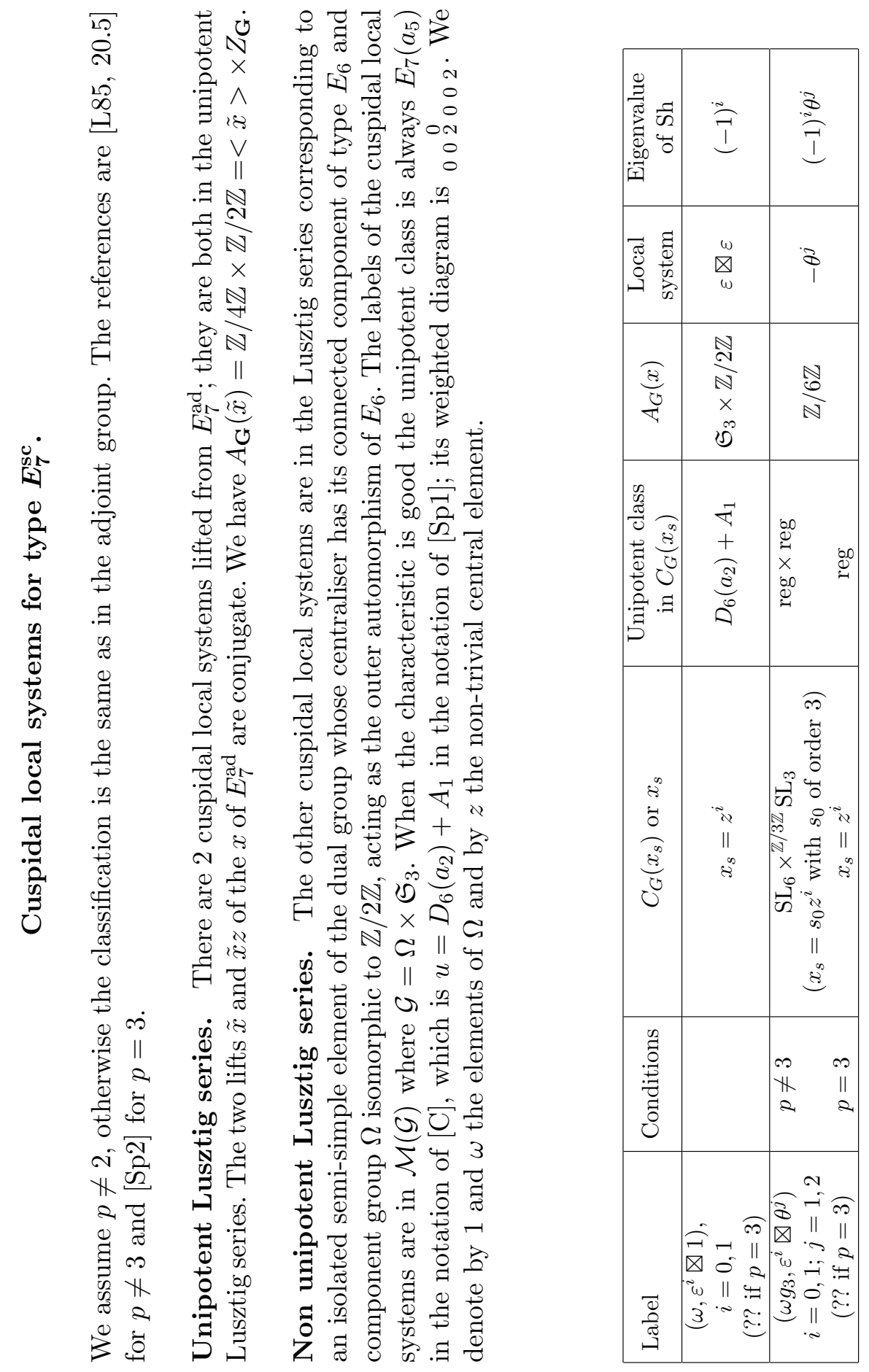




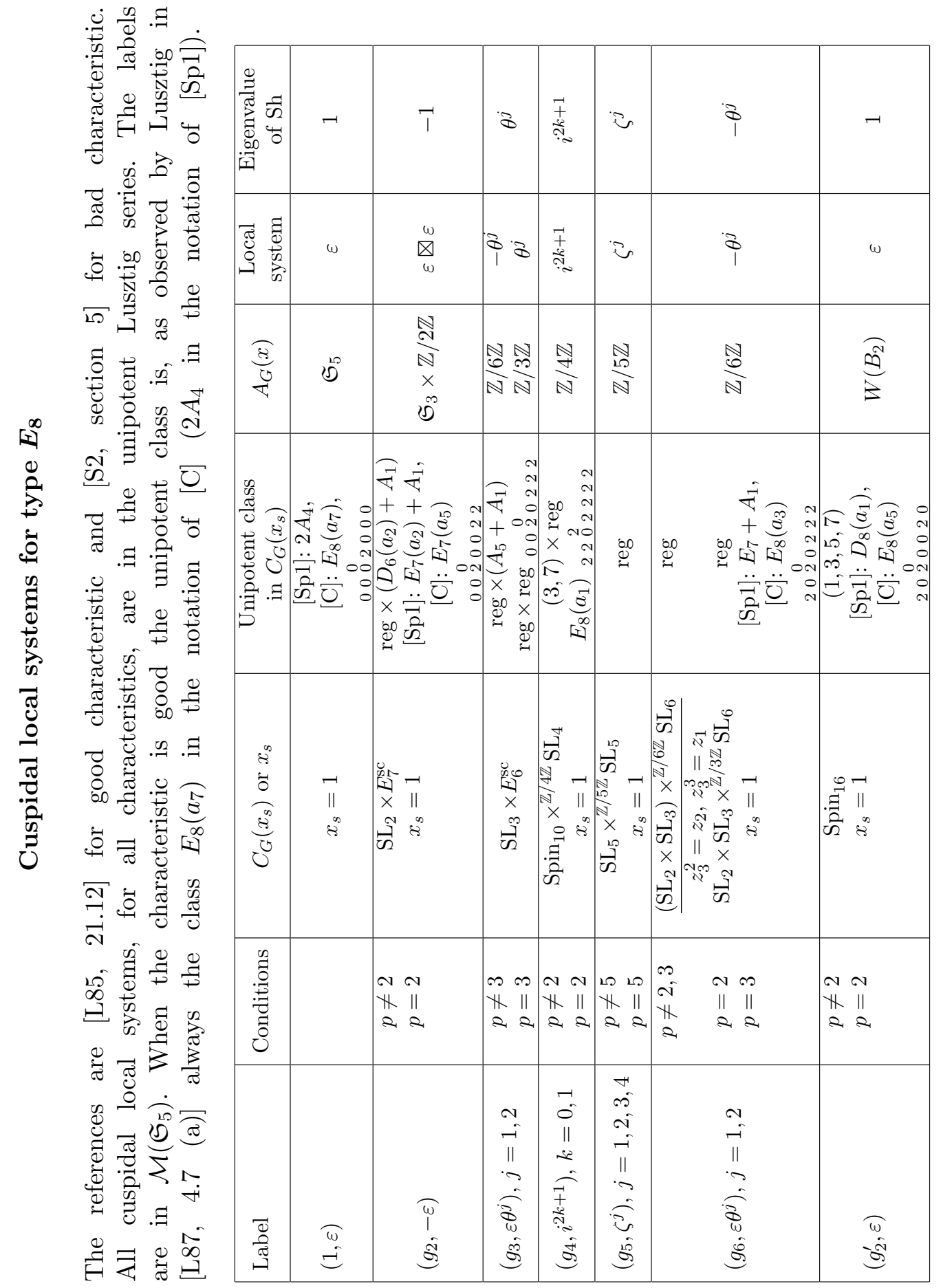


On Character Sheaves and Characters of Reductive Groups...

509

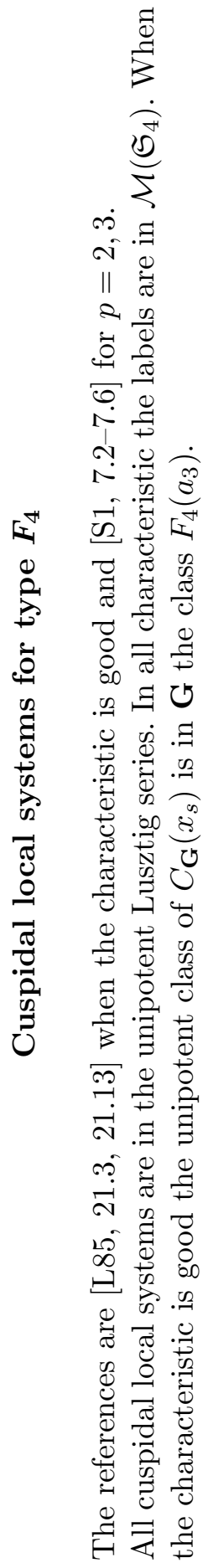

\begin{tabular}{|c|c|c|c|c|c|}
\hline 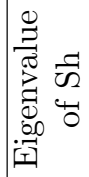 & - & † & - & 3 & 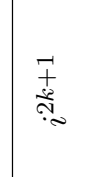 \\
\hline 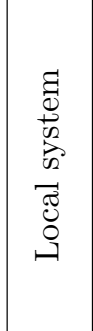 & $\omega$ & $\omega$ & 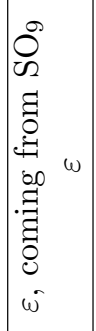 & 3 & 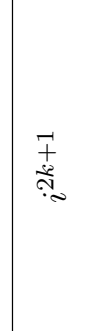 \\
\hline$\underset{\overparen{E}}{\overparen{E}}$ & (1) & $\stackrel{\stackrel{N}{N}}{\mathbb{N}}$ & $\frac{\widehat{D}}{B}$ & $\frac{\mathbb{N}}{\mathbb{N}}$ & $\frac{\mathbb{N}}{\mathbb{N}}$ \\
\hline 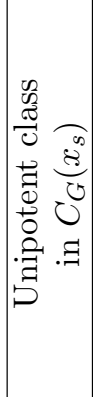 & 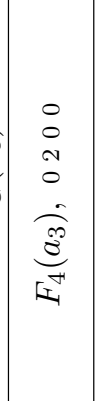 & 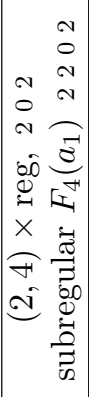 & 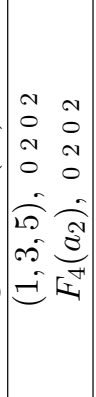 & 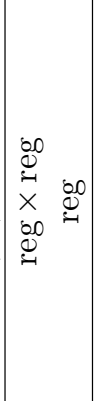 & 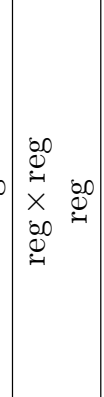 \\
\hline 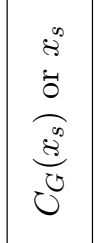 & $\begin{array}{l}\overrightarrow{\|} \\
\| \\
\infty\end{array}$ & 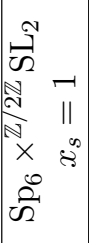 & 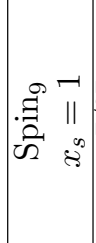 & 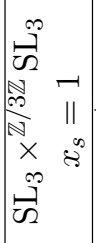 & 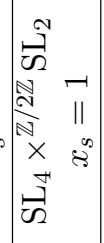 \\
\hline 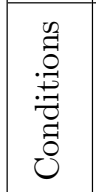 & $\begin{array}{ll}N & 2 \\
W & 11 \\
2 & 2\end{array}$ & $\begin{array}{ll}N & N \\
W & 11 \\
2 & 2\end{array}$ & $\begin{array}{cc}N & 2 \\
W & 11 \\
2 & 2\end{array}$ & $\mid \begin{array}{ll}\infty & \infty \\
1 & 11 \\
2 & 2\end{array}$ & $\begin{array}{ll}N & N \\
W & 11 \\
2 & 2\end{array}$ \\
\hline 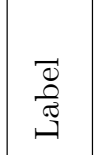 & 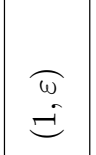 & $\begin{array}{c}\widehat{\omega} \\
\hat{S}\end{array}$ & $\begin{array}{l}\widehat{\omega} \\
\hat{\theta}\end{array}$ & 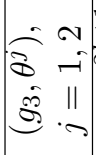 & 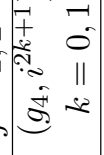 \\
\hline
\end{tabular}




\section{References}

[AA] P.N. Achar, A.-M. Aubert, "Localisations de faisceaux caractères", Adv. Math. (2010), doi:10.1016/j.aim.2010.02.002.

[B] C. Bonnafé, "Sur les caractères des groupes réductifs finis à centre non connexe : applications aux groupes spéciaux linéaires et unitaires" Asrérisque 306 (2006).

[C] R. Carter, "Finite groups of Lie type: conjugacy classes and complex characters", Wiley (1985).

[DLM1] F. Digne, G.I. Lehrer and J. Michel, "The characters of the group of rational points of a reductive group with non-connected centre" Crelle's Journal 425 (1992), 155-192.

[DLM2] F. Digne, G.I. Lehrer and J. Michel, "On Gel'fand-Graev characters of reductive groups with disconnected centre", Crelle's Journal 491 (1997), 131-148.

[DLM3] F. Digne, G.I. Lehrer and J. Michel, "The space of unipotently supported class functions" Journal of Algebra, 60 (2003) 111-137

[DM1] F. Digne and J. Michel, "Fonctions $L$ des variétés de DeligneLusztig et descente de Shintani. (French) [L-functions of DeligneLusztig varieties and Shintani descent]", Mém. Soc. Math. France (N.S.) No. 20 (1985).

[DM2] F. Digne and J. Michel, "On Lusztig's parametrization of characters of finite groups of Lie type", Astérisque No. 181-182 (1990), 6, $113-156$.

[G] M. Geck, "Character sheaves and generalized Gelfand-Graev characters", Proc. London Math. Soc. 78 (1999), 139-166.

[K2] N. Kawanaka, "Generalized Gelfand-Graev representations of exceptional simple algebraic groups over a finite field I", Inventiones 84 (1986), 575-616.

[L79] G. Lusztig, "On the reflection representation of a finite Chevalley group" LMS Lecture Notes 34 (1979), 325-337.

[L79b] G. Lusztig, "A class of irreducible representations of a Weyl group" Indag. Math. 41 (1979) 323-335; Chevalley group" LMS Lecture Notes 34 (1979), 325-337. 
[L84] G. Lusztig, "Characters of reductive groups over a finite field", Annals of Math. Studies 107 (1984) Princeton University Press.

[L84b] G. Lusztig, "Intersection cohomology complexes on a reductive group", Inventiones 75 (1984), 205-272.

[L85] G. Lusztig, "Character Sheaves", Advances in Math. 56 (1985), 193-237, 57 (1985), 226-265 and 266-315, 59 (1986), 1-63, 61 (1986), 103-165.

[L86] G. Lusztig, "On the character values of finite Chevalley groups at unipotent elements" Journal of Algebra, 104 (1986) 146-194

[L87] G. Lusztig, "Introduction to character sheaves" Proc. Symposia in Pure Math. 47 (1987) 165-180.

[L92] G. Lusztig, "Remarks on computing irreducible characters" Journal of AMS. 5 (1992), 971-986.

[L92b] G. Lusztig, "A unipotent support for irreducible representations", Advances in Math. 94 (1992), 139-179.

[L12] G. Lusztig, "On the cleanness of cuspidal character sheaves", Moscow Math. J. 12 (2012) 621-631.

[L12b] G. Lusztig, "Families and Springer's correspondence", arXiv: 1201.5593 (2012).

[L12c] G. Lusztig, "Restriction of a character sheaf to conjugacy classes $"$, arXiv: 1204.3521 (2012).

[LS] G. Lusztig and N. Spaltenstein, "On the generalized Springer correspondence for classical groups", Adv. studies in pure math. 6 (1985), 289-316.

[Os] V. Ostrik, "A remark on cuspidal local systems", Adv. Math. 192 (2005), no. 1, 218-224.

[S1] T. Shoji, "Character sheaves and almost characters of reductive groups", Advances in math. 111 (1995), 244-313.

[S2] T. Shoji, "Character sheaves and almost characters of reductive groups, II", Advances in math. 111 (1995), 314-354.

[S3] T. Shoji, "Lusztig's conjecture for finite special linear groups" Representation Theory 10 (2006), 164-222 
[S4] T. Shoji, "Lusztig's conjecture for finite classical groups with even characteristic" Contemp. Math. 478 (2009), 207-236.

[Sp1] N. Spaltenstein, "Classes unipotentes et sous-groupes de Borel", Lecture Notes in Mathematics 946, Springer (1982).

[Sp2] N. Spaltenstein, "On the generalized Springer correspondence for exceptional groups", Advanced studies in pure math. 6 (1985), 317338.

[T] J. Taylor "On unipotent supports of reductive groups with a disconnected centre" J. of Algebra 391 (2013) 41-61.

[W] J.-L. Waldspurger, "Une conjecture de Lusztig pour les groupes classiques", Mém. Soc. Math. France 96 (2004).

François Digne

Laboratoire Amiénois de Mathématique Fondamentale et Appliquée, CNRS UMR 7352, Université de Picardie-Jules Verne, 80039 Amiens Cedex France.

E-mail: digne@u-picardie.fr

Gustav Lehrer

School of Mathematics and Statistics, University of Sydney, NSW 2006, Australia.

E-mail: gustav.lehrer@sydney.edu.au

Jean Michel

Institut de Mathématiques de Jussieu - Paris rive gauche, Université Denis Diderot, Bâtiment Sophie Germain, 75013, Paris France.

E-mail: jean.michel@imj-prg.fr 